\title{
Methodologies in the Analysis of Phenolic Compounds in Roselle (Hibiscus sabdariffa L.): Composition, Biological Activity, and Beneficial Effects on Human Health
}

\author{
Bety W. Hapsari ${ }^{1,2}$, Manikharda ${ }^{2}$ and Widiastuti Setyaningsih ${ }^{2, *}$ (D) \\ 1 National Standardization Agency of Indonesia-BSN, Jalan MH Thamrin No. 8 Kebon Sirih, \\ Central Jakarta 10340, Indonesia; bety.hapsari@mail.ugm.ac.id \\ 2 Department of Food and Agricultural Product Technology, Faculty of Agricultural Technology, \\ Universitas Gadjah Mada, Jalan Flora, Depok, Sleman, Yogyakarta 55281, Indonesia; manikharda@ugm.ac.id \\ * Correspondence: widiastuti.setyaningsih@ugm.ac.id; Tel.: +62-274-549-650
}

check for

updates

Citation: Hapsari, B.W.; Manikharda; Setyaningsih, W. Methodologies in the Analysis of Phenolic Compounds in Roselle (Hibiscus sabdariffa L.):

Composition, Biological Activity, and Beneficial Effects on Human Health. Horticulturae 2021, 7, 35. https:// doi.org/10.3390/horticulturae7020035

Academic Editors: Douglas D. Archbold and Silvana Nicola

Received: 31 January 2021

Accepted: 19 February 2021

Published: 23 February 2021

Publisher's Note: MDPI stays neutral with regard to jurisdictional claims in published maps and institutional affiliations.

Copyright: (c) 2021 by the authors. Licensee MDPI, Basel, Switzerland. This article is an open access article distributed under the terms and conditions of the Creative Commons Attribution (CC BY) license (https:/ / creativecommons.org/licenses/by/ $4.0 /)$.

\begin{abstract}
Roselle (Hibiscus sabdariffa L.), as an edible flower, has long provided an array of positive effects on human health. This benefit is a result of phenolic compounds that are naturally present mainly in the calyx. Plentiful medicinal remedies and functional foods based on this flower are available worldwide, as supported by the studies of phenolic compounds in recent decades. This paper aims to provide a comprehensive review of the composition, biological activity, and beneficial effects on human health of phenolic compounds in roselle. This review was performed in accordance with the Preferred Reporting Items for Systematic reviews and Meta-Analyses (PRISMA) guidelines. A structured search in the published literature for phenolics compositions in roselle was required prior to the evaluation on the validity of the reported analytical methods. Reliable identification and quantification of phenolic compounds in roselle can be achieved by employing the proper extraction and separation methods. With ample alternative analytical methods discussed here, this review provided an aid for comprehending and selecting the most appropriate method for a particular study. The applications of the analytical methods highlighted indicated that phenolic acids, flavonoids, and their derivatives have been identified and quantified in roselle with a range of biological activities and beneficial effects on human health. It was also disclosed that the composition and concentration of phenolic compounds in roselle vary due to the growth factors, cultivars, and environmental influence. Finally, apart from the research progress carried out with roselle during the last ten years, this review also proposed relevant future works.
\end{abstract}

Keywords: edible flower; analytical method; functional food; health benefit; flavonoids

\section{Introduction}

The trend of healthy lifestyles has changed society's eating habits. The population prefers to consume foods that provide additional health benefits in addition to a consideration of sensory attributes, such as flavor, taste, and appearance. Hence, the market for functional foods is facing increasing demand, particularly when enhanced by the attractiveness of edible flowers [1]. One of the most widely utilized edible flowers is roselle (Hibiscus sabdariffa L.) [2-4].

The primarily utilized part of the roselle plant is the floral calyx, which is medicinally useful. Traditionally, roselle is consumed as a healthy drink by infusing its petals and served either cold or hot [5]. Presently, roselle has also been incorporated in diverse applications, including foods, cosmetics, and pharmaceuticals [6-8]. To further incorporate the flower in industrial product development, research into roselle calyces has notably increased within the last decade [9-11]. Studies have reported that the calyx contains a number of phytochemical compounds, and phenolics are considered to be the most influential in providing various health benefits [12]. Some of the phenolic compounds found in 
roselle calyces are flavonoids and phenolic acids $[13,14]$. These compounds have been confirmed to provide health benefits, such as lipid-lowering activity and antihypertensive, antibacterial, antidiabetic, antioxidant, nephroprotective, hepatoprotective, renal/diuretic effects, and anti-cholesterol effects [15].

Previous reviews and meta-analysis studies on roselle have mostly reported the functional properties ensuring health benefits for the population $[13,14,16-20]$. Corresponding to the growing interest in roselle and the current challenges to developing new healthy products [21], beneficial compounds should be determined by reliable analytical methods [22]. The application of those analytical methods will provide opportunities in many related studies, including analyses of the composition of beneficial compounds in roselle, and further evaluate the biological activity and beneficial effects of phenolic compounds in roselle on human health.

\section{Methods}

\subsection{Data Sources}

This systematic review focused on current studies of advances in the analysis of phenolic compounds in roselle that were further applied to determine the composition, biological activity, and beneficial effects on human health. The information source was the Scopus database via the keywords of "Hibiscus sabdariffa" and "roselle", searched separately or combined in a title, abstract, and keyword. The information was collected from documents published from January 2010 to January 2021.

\subsection{Inclusion and Exclusion of Data and Data Quality}

This review was performed in accordance with the Preferred Reporting Items for Systematic reviews and Meta-Analyses (PRISMA) guidelines, as presented in Figure 1. The document selection had to meet the (1) inclusion criteria: (a) identify and quantify the phenolics and their derivatives and (b) evaluate the biological activity and beneficial effects on human health and (2) exclusion criteria: (a) published in a non-English language, (b) non-research article, and (c) did not measure roselle calyx tissue.

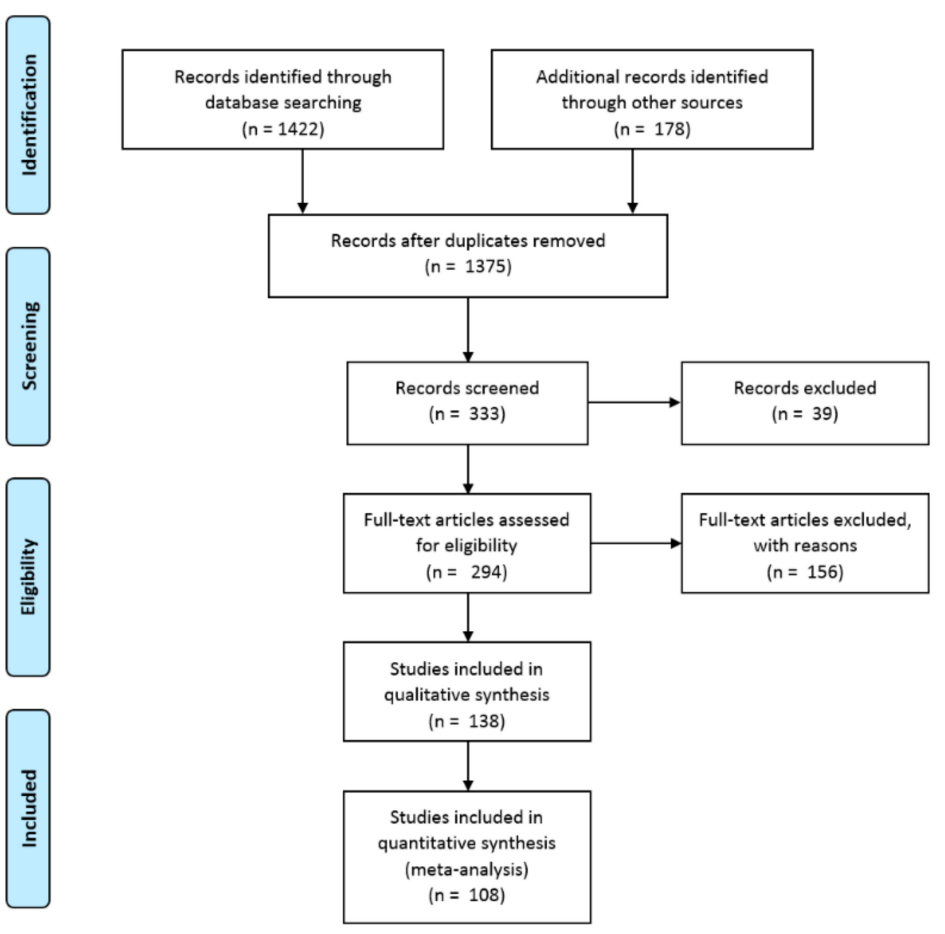

Figure 1. Preferred Reporting Items for Systematic reviews and Meta-Analyses (PRISMA) flow diagram to summarize the search and inclusion/exclusion procedure. 


\section{Roselle Overview}

It is assumed that the roselle plant was first recognized before $4000 \mathrm{BC}$ in Africa, specifically in Western Sudan [23]. A historical study in 1911 by Wester disclosed that the plant was allegedly brought from India to Turkey and named "Sabdariffa". The name is claimed to be adopted from a Turkish word [24]. Later on, roselle was phylogenetically classified in the genus Hibiscus and the Malvaceae family. As civilization expanded, roselle was widely grown and cultivated in tropical and subtropical countries, such as South and Southeast Asia, Africa, and Central America [25]. H. sabdariffa var. Sabdariffa and H. sabdariffa var. Altissimac Wester are two types of roselle that are commonly cultivated worldwide for economic purposes [24].

Roselle plants, characterized as an annual shrub, were reported as having erect reddish stems, branching, and growth up to $3.5 \mathrm{~m} \mathrm{[26].} \mathrm{The} \mathrm{leaves} \mathrm{sprout} \mathrm{alternately} \mathrm{on} \mathrm{the} \mathrm{stem}$ and are usually around 7.5 and $12.5 \mathrm{~cm}$ in length [24]. The flower's positions are axillary or terminal and $8-10 \mathrm{~cm}$ in diameter. The flower color varies from white to pale yellow, with a dark red spot at each petal base. It is described as having a stout fleshy calyx at the base 1 to $2 \mathrm{~cm}$ wide and enlarging to $3-3.5 \mathrm{~cm}$. When the flower matures, it will turn fleshy and bright red [14]. The plant requires an adequate amount of water and sunlight throughout the day; thus, it can easily grow in areas with tropical climates [26]. Roselle can be planted when the seedlings are 10-15 days old. During the initial growth period, roselle needs $13 \mathrm{~h}$ of sun exposure per day and a temperature no lower than $20^{\circ} \mathrm{C}$ at night. The amount of rainfall required is around $130-250 \mathrm{~mm}$ per month, or sufficient watering is needed. The time needed for roselle to be harvested is approximately 4 to 5 months, and the optimum period of the year to start planting is December to January [23,24].

The harvest time and postharvest handling, especially the drying procedure, affect the physical and chemical properties of roselle [23]. Other factors, such as the specific cultivar and environmental conditions, also strongly influence the floral properties [27,28]. In fact, these two factors define the level of mineral and phytochemical compounds in the calyces $[29,30]$. The concentration of the phenolic compounds and the antioxidant activity of the calyx, for instance, can be altered by increasing the $\mathrm{CO}_{2}$ content in the atmosphere of the growing areas of roselle plants [31]. Hence, to achieve a phenolic-rich calyx providing significant antioxidant activity, the aforementioned factors must be modified or optimized.

Phenolic compounds naturally found in roselle are phenolic acids and flavonoids. The phenolic acids described in roselle include neochlorogenic acid, chlorogenic acid, cryptochlorogenic acid, methyl chlorogenate, coumaroylquinic acid, dihydroferulic acid-4$\mathrm{O}$-glucuronide, ethyl chlorogenate, and 5-O-caffeoyl shikimic acid. At least 95 flavonoids have been identified in the roselle calyx [32]. Among the identified flavonoids, anthocyanins are most abundant and mainly consist of delphinidin-3-O-sambubioside and cyanidin-3-O-sambubioside [33]. Frequently reported flavonoids also include quercetin-3glucoside, methyl epigallocatechin, myricetin, quercetin, and kaempferol. Other important compounds like organic acids are also presented in roselles, such as hydroxycitric acid, hibiscus acid, hibiscus acid hydroxyethyl ester, and hibiscus acid dimethyl ester [12].

The compounds mentioned above have been confirmed through a number of former studies to provide diverse health benefits. The level of total phenolics has been positively correlated with the antioxidant activity [34-37]. Specific types of compounds, i.e., phenolic acids (ferulic and chlorogenic acids), organic acids (citrate derivatives), and flavonoids (kaempferol), have demonstrated antihypertensive properties [38]. In addition, hydroxycitric acid from a roselle calyx could be a weight loss agent [39], gossypetin, an antidepressant [40], and hibiscus acid, an antimicrobial compound [41]. Having these beneficial effects, the consumption and demand for roselle have continued to increase over the last decade.

Roselle is practically consumed as a hot or cold infusion of the flower petals into a liquid or may further be processed into a fermented drink [5]. Besides that, the calyces are used as the primary material to prepare roselle syrup, jam, jelly, and other food industry products. Furthermore, the water extract of the calyx can be incorporated with other food 
material into refreshing beverages, vitamin and mineral supplements, yogurt, and hard rock candy [21].

The young leaves and tender stems of roselle are frequently consumed raw or cooked, including in salads or curry condiments. The seeds are roasted or ground for cooking or oil extraction, while the grouts are used as soup ingredients $[7,23]$.

The consumption of roselle has been reported to reduce the risk of various degenerative diseases, such as cardiovascular disease, diabetes mellitus, and obesity. The health benefits of roselle consumption have been confirmed through both in vitro and in vivo studies [42-44]. The recognized health-boosting mechanisms include lowering significantly the levels of total and low-density lipoprotein (LDL) cholesterol, systolic blood pressure, diastolic blood pressure, and fasting plasma glucose [17-20]. Increasingly acknowledged for its health benefits, roselle has been used in traditional medicine around the globe [16,39].

\section{Analytical Methods for Phenolic Compounds in Roselle}

A reliable analytical method is required before performing related studies to take advantage of the phenolic compounds in roselle and its derived products. Valid identification and quantification by an appropriate analytical method could provide the right composition of phenolic compounds in the matrix. The selection of the analytical methods, including extraction, separation, and detection, depends on the characteristics of the sample matrices and target analytes. The development of alternative analytical methods and determination of the phenolic compounds in roselle calyx are discussed in this section.

\subsection{Sample Preparation}

The initial step in most analytical procedures is sample preparation to convert a real matrix into a sample suitable for analysis. It is crucial when handling complex matrices of samples containing lipids, proteins, carbohydrates, or other constituents that may interfere with the target analyte. In this case, a method of separation or purification of nontarget compounds is required to remove the impurities, and extraction is frequently performed for sample preparation. However, special conditions might restrict the extraction of fresh samples; thus, additional sample preparation, viz., a drying procedure, is often required [45,46]. Conventional extraction, such as Soxhlet, maceration, percolation, and infusion, has been routinely used due to its simplicity $[45,47]$. With technological developments, some advanced extraction methods have been developed, including microwave-assisted extraction (MAE), ultrasound-assisted extraction (UAE), pressurized liquid extraction (PLE), and supercritical fluid extraction (SFE) [48]. These newer methods are beneficial in reducing the extraction time and solvent volume [45]. These novel extraction methods employed for phenolic compound extraction from the roselle calyx are compiled in Figure 2.

The most frequently used methods to extract phenolic compounds from roselle calyx are maceration, UAE, MAE, and SFE. Table 1 compiles alternatives of the extraction methods for phenolic compounds in different roselle cultivars. The selection of the extraction method depends on the type of phenolics and sample matrices [49]. Meanwhile, the method development requires the optimization of factors that likely affect the extraction efficiency, such as solvent composition, particle size, solid-to-solvent ratio, extraction temperature, and time $[45,47,50]$.

The most commonly used extraction solvents for phenolic compounds from roselle are distilled or purified water [51-61], ethanol [10,32,62,63], methanol [38,64,65], and mixtures of the aforementioned solvents with acids, i.e., hydrochloric acid [3], formic acid [58,66], and citric acid-based deep eutectic solvent (DES) [67]. The acidification treatment of solvent in the extraction procedure has resulted in higher functional properties of the phenolics $[3,8,68]$. Furthermore, as an acidic environment maintains the stability of anthocyanins, a complete recovery for these analytes can be achieved by utilizing acidic solvents [69-71]. In contrast, the optimum solvent composition for recovering flavonoids was $50 \%$ ethanol [64]. Another study determined that $80 \%$ methanol could extract flavonoids with the highest angiotensin-converting enzyme (ACE) inhibitory activity [38]. 
The extraction recovery was also affected by the particle size of the sample [55,72]. A study on the particle size of roselle calyx powder $(0-180 \mu \mathrm{m}, 180-212 \mu \mathrm{m}, 212-315 \mu \mathrm{m}$, and $\geq 315 \mu \mathrm{m}$ ) reported that the highest recovery of phenolic compounds producing antioxidant activity was obtained from a sample with particle sizes of 212-315 $\mu \mathrm{m}$ [72]. This result was similar to a former report that particle sizes of 250-177 $\mu \mathrm{m}$ recovered higher phenolics than the smaller particle sizes 177-150 and 150-74 $\mu \mathrm{m}$ [55]. Specifically, for anthocyanins, a higher recovery was obtained by extracting calyx powder with particle sizes smaller than $250 \mu \mathrm{m}$ [73].

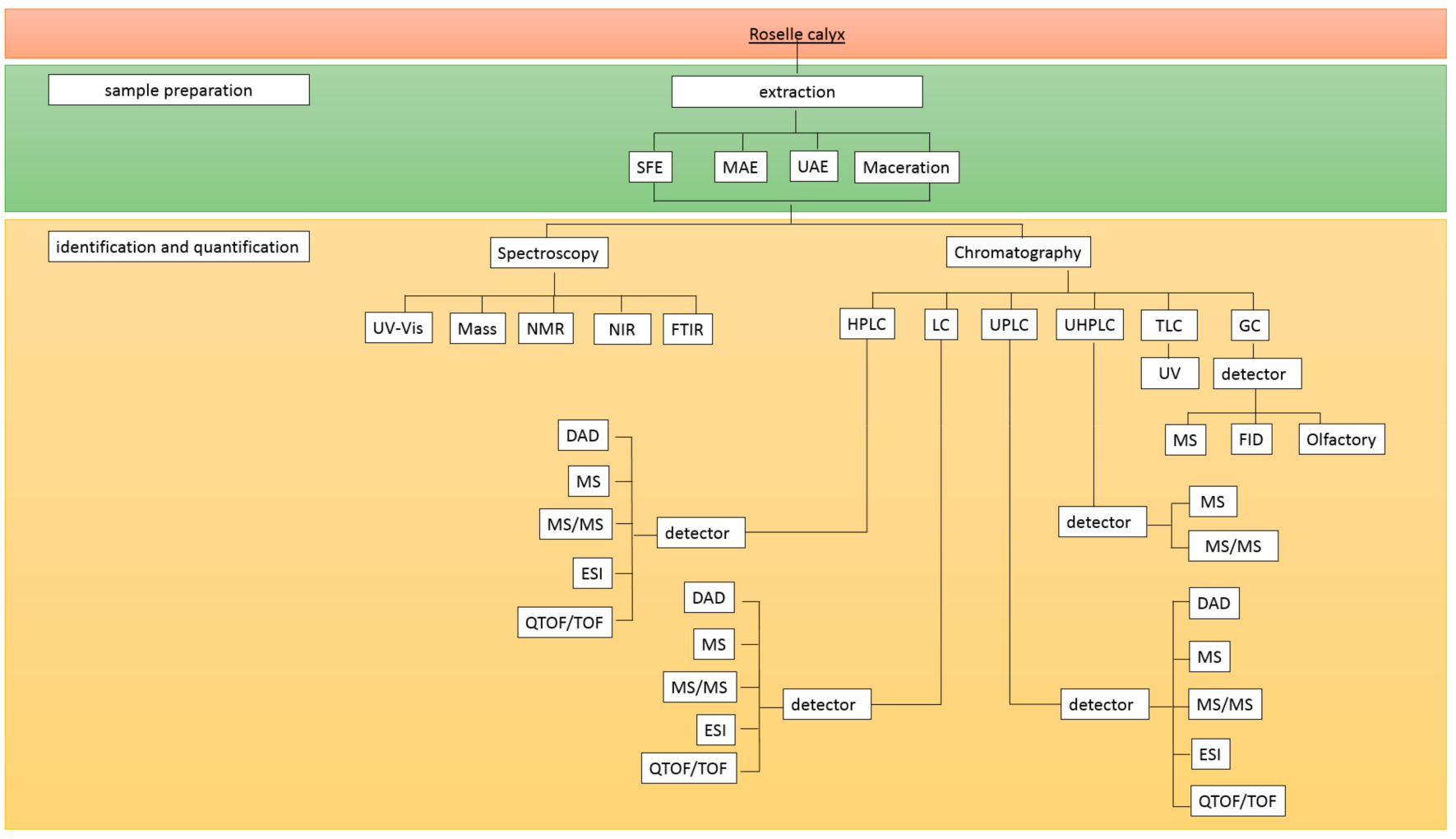

Figure 2. Diagram of the analytical methods to determine the phenolic compounds in roselle. Abbreviations: SFE: Supercritical Fluid Extraction, MAE: Microwave-Assisted Extraction, UAE: Ul-tra-sound-Assisted-Extraction, UV-Vis: ultra-violet-visible, NMR: nuclear magnetic resonance, NIR: near-infrared spec-troscopy, FTIR: Fourier Transform Infrared Spectrometer, HPLC: high-performance liquid chromatography, LC: liquid chromatography, UPLC: ultra-performance liquid chromatography, UHPLC: ultrahigh-performance liquid chromatog-raphy, TLC: thin-layer chromatography, GC: gas chromatography, DAD: diode array detector, PDA: photodiode array detector, MS: mass spectrophotometry, ESI: electrospray ionization, TOF/QTOF: quadrupole time-of-flight, FID: flame ionization detector.

A higher recovery can also be achieved with an optimum tissue-to-solvent ratio when extracting the total phenolics, flavonoids, and anthocyanins in roselle $[10,11,52]$ with higher antioxidant activities [74]. For instance, a tissue-to-solvent ratio of 1:14 was reported in a study providing extracts with a higher total phenolic compound (TPC) value than those at lower (1:10) or higher (1:16 and 1:18) ratios [11]. Furthermore, for anthocyanin extraction, increasing the tissue-to-solvent ratio from 1:2 to 1:8 increased the total anthocyanin compound (TAC) value from $87.47 \mathrm{mg} / \mathrm{L}$ to $158.56 \mathrm{mg} / \mathrm{L}$; however, the value decreased at a ratio of 1:10 [62]. A larger amount of extraction solvent facilitated the dissolution of the analyte as a result of a significant mass transfer, thus accelerating the diffusion. Before the process reached its equilibrium, the extraction process remained unchanged [10].

The extraction rate was also regulated by the extraction temperature and time. A higher temperature led to a higher diffusion rate, extracting a greater amount of target compounds [52]. While increasing the extraction time, significant increases in the TPC, TAC, 
and antioxidant capacity were observed $[10,22,75]$. A single-factor experiment evaluating the temperature reported a range of $60-80^{\circ} \mathrm{C}$ as the appropriate extraction temperature for the total flavonoid compound (TFC), while temperatures higher than $60^{\circ} \mathrm{C}$ were suitable for TPC extraction [75]. However, a longer extraction time with a higher temperature also tended to increase the degradation rate of phenolics due to exposure to a favorable environment [76]. When the degradation rate was higher than the extraction rate, the extracted thermolabile flavonoids could decrease [77].

Due to the influence of various extraction factors, both single and concurrent optimization were performed to develop the extraction methods for phenolic components in roselle, including maceration $[6,52,76,78]$, UAE $[9,54,79]$, MAE [11,67,77], and SFE [12,80].

\subsubsection{Maceration}

Maceration is the most practical solid-liquid extraction (SLE) widely used conventional extraction method to recover phenolic compounds from roselle calyx $[3,6,34,52,55,57-60,62,63,65,75,76,78,81-83]$. This extraction method relies on both conductive and convective processes to heat the product [49]. Hence, the extraction recovery is greatly influenced by the temperature. The maceration method was evaluated with temperatures ranging from 25 to $180{ }^{\circ} \mathrm{C}$ in a number of studies listed in Table 1. It was subsequently found that the optimum temperature ranged from 40 to $60{ }^{\circ} \mathrm{C}[6,52,55,57-60,62,63,65,74-76,78,81,82]$. Nonetheless, a lower temperature is more appropriate to recover a higher level of anthocyanins [39].

In addition to the extraction temperature in question, the extraction time is also considered an influential factor in maceration. The effect could be evaluated during a mass transfer kinetic study where the more prolonged the extraction time, the higher the level of the extracted analyte. However, unnecessary extended extraction times could lead to a lower recovery when the degradation rate of the thermolabile phenolics is higher than the extraction rate. The extraction times reported in some studies for the maceration method ranged from $10 \mathrm{~min}$ to $72 \mathrm{~h}[58,65]$, but it commonly takes place from 1 to $4 \mathrm{~h}$. A 20-min maceration at $60^{\circ} \mathrm{C}$ has been proven to reach a complete recovery for anthocyanins from roselle provided water was used as the optimized extraction solvent [76]. Apart from selecting the right solvent for a certain analyte, the ratio between the sample and solvent must also be optimized when developing an efficient extraction. In maceration, the ratio of sample-to-solvent ranged from 1:4 to 1:100 [58,81].

\subsubsection{Ultrasound-Assisted-Extraction (UAE)}

Among the alternative modern extraction methods (Table 1), ultrasound-assisted extraction (UAE) is the most frequently used for phenolic extraction from roselle. The extraction is aided by ultrasonic waves at specific frequencies and amplitudes, producing cavitation bubbles. Provided that the ultrasound-induced cavitation bubbles collapse, high shear stresses in the fluid are created, breaking the cell walls and triggering analyte release to the solvent [48]. This mechanism can be appropriately set by optimizing the pulse duty cycle and the ultrasonic power. Other UAE factors, such as extraction time, temperature, solvent, and tissue-to-solvent ratio, also influence the process [49].

The extraction conditions evaluated in various studies of phenolics in roselle for $\mathrm{UAE}$ include extraction time $(26-45 \mathrm{~min})$, temperature $\left(25-65^{\circ} \mathrm{C}\right)$, ultrasound power $(300 \mathrm{~W})$, and frequency $(40 \mathrm{kHz})$. UAE optimization for flavonoid extraction resulted in an extraction setting as follows: $25 \%$ ethanol as the extraction solvent at $65^{\circ} \mathrm{C}$ in $45 \mathrm{~min}$. This UAE condition provided extracts with a higher level of quercetin hexoside, quercetin, myricetin, and anthocyanin compared to the maceration-produced extract [9]. In addition, the levels of extracted total phenolics and anthocyanins (delphinidin-3-O-sambubioside and cyanidin-3$\mathrm{O}$-sambubioside) were two-to-four times higher in UAE over the conventional heat-assisted SLE $[9,79]$. Furthermore, applying the same extraction temperature and solvent, UAE can have a shorter extraction time than the conventional SLE method, even with higher levels of bioactive compounds in the extract [10]. 


\subsubsection{Microwave-Assisted Extraction (MAE)}

The unique characteristic of microwave-assisted extraction (MAE) is the heating mechanism utilizing radiation at a frequency ranging from $300 \mathrm{MHz}$ to $300 \mathrm{GHz}$. The sample is heated through dielectric heating (continuous dipole rotation) and frictional resistance to the ion flow. Both processes trigger an increase in the heating energy to achieve an efficient extraction [47]. Besides the radiation energy, the MAE method is also affected by the extraction time, temperature, solvent composition, and tissue-to-solvent ratio [49].

The main advantage of MAE over UAE is that the system allows multiple extractions at a time. MAE facilitates up to 24 extraction vessels that can be used in a single procedure. Hence, the actual extraction time per sample in one MAE batch running for multiple vessels can provide a fast extraction method for routine analysis. A 3-min extraction at $60^{\circ} \mathrm{C}$ with $500-\mathrm{W}$ microwave power could yield 70.53-mg gallic acid equivalents (GAE)/g roselle calyx [11]. In comparison, applying the optimized flavonoid extraction (450-W microwave power, $40{ }^{\circ} \mathrm{C}$ extraction temperature, $52 \%$ ethanol, and $15: 1$ solvent-to-sample ratio) results in a TFC value of $94.32-\mathrm{mg}$ quercetin equivalents (QE)/g roselle calyx in merely $4 \mathrm{~min}$ of extraction time [32]. Contrasted with conventional SLE, MAE consumes one-third of the extraction time by maceration while producing extract with a higher antioxidant activity [8].

\subsubsection{Supercritical Fluid Extraction (SFE)}

Supercritical fluid extraction (SFE) utilizes a gas above the critical point, exhibiting liquid-like properties while still maintaining the nature of a gas [84]. Hence, the most influential SFE factors are the extraction solvent, pressure, and temperature [85]. SFE improved the collection of hibiscus acid and its derivatives from roselle after maceration. In the extraction of phenolic compounds in roselle, applying extraction conditions at $50{ }^{\circ} \mathrm{C}$ and 250 bar with $16.7 \%$ ethanol, SFE provided total phenolic compounds (TPC) of 113-mg GAE/g extract [12]. Another study reported that 8.63-mg GAE/g dried roselle was obtained by SFE at $343.15 \mathrm{~K}$ with $24 \mathrm{MPa}$ in $2.5 \mathrm{~h}$. An increase in the extraction pressure and temperature would raise the amount of extracted phenolic compounds [80].

\subsubsection{Miscellaneous Extraction Techniques}

Apart from the extraction methods mentioned above, other techniques to recover phenolic compounds from roselle calyx are pressurized hot water extraction and enzymeassisted extraction. To enhance the purity of the phenolic compounds in a roselle extract, fractionation and purification techniques were used for the isolation of the compounds. This approach included the use of polyamide column chromatographic purification [86], a combination of macroporous resins and a $\mathrm{C}_{18}$ Sep-Pak cartridge [87], a single-step process fractionation using a Sep-Pak $\mathrm{C}_{18}$ cartridge [71], and semipreparative high-performance liquid chromatography (HPLC) purification [88].

Table 1. The extraction methods used for roselle phenolic compounds and antioxidant activities. Abbreviations: TPC: total phenolic compound, TFC: total flavonoid compound, TAC: total anthocyanin compound, UAE: ultrasound-assisted extraction, MAE: microwave-assisted-extraction, SFE: supercritical fluid extraction, GAE: gallic acid equivalents, Cy-3GE: cyanidin-3-glucoside equivalents, CE: catechin equivalents, QE: quercetin equivalents, ACEI: angiotensin-converting enzyme inhibitory, FRAP: ferric ion-reducing antioxidant power, ORAC: oxygen radical absorbance capacity, DPPH: 1-diphenyl-2-picrylhydrazyl, and ABTS: 2,2'-azino-bis-3-ethylbenzthiazoline-6-sulphonic acid.

\begin{tabular}{cccccc}
\hline $\begin{array}{c}\text { Country of } \\
\text { Origin }\end{array}$ & $\begin{array}{c}\text { Extraction } \\
\text { Method }\end{array}$ & Solvent & Extraction Condition & $\begin{array}{c}\text { Phenolics and Antioxidant } \\
\text { Activity }\end{array}$ & Ref \\
\hline Vietnam & maceration & $50 \%$ ethanol & $\begin{array}{c}\text { sample:solvent ratio } 1: 7.7 \\
\text { extraction temperature } 56.9^{\circ} \mathrm{C} \\
\text { extraction time } 33.29 \mathrm{~min}\end{array}$ & TAC (186.01 mg/L) \\
\hline Mexico & maceration & purified water & $\begin{array}{c}\text { extraction temperature } 95^{\circ} \mathrm{C} \\
\text { extraction time } 20 \mathrm{~min} \\
\text { particle size } 250-177 \mu \mathrm{m}\end{array}$ & $\begin{array}{c}\mathrm{TPC}(14.10 \mathrm{mg} \mathrm{GAE} / \mathrm{g}), \mathrm{TFC} \\
(10.18 \mathrm{mg} \mathrm{CE} / \mathrm{g}), \mathrm{and} \mathrm{TAC} \\
(5.68 \mathrm{mg} \mathrm{Cy-3-GE} / \mathrm{g})\end{array}$ \\
\hline
\end{tabular}


Table 1. Cont.

\begin{tabular}{|c|c|c|c|c|c|}
\hline $\begin{array}{l}\text { Country of } \\
\text { Origin }\end{array}$ & $\begin{array}{l}\text { Extraction } \\
\text { Method }\end{array}$ & Solvent & Extraction Condition & $\begin{array}{c}\text { Phenolics and Antioxidant } \\
\text { Activity }\end{array}$ & Ref \\
\hline Saudi Arabia & maceration & water & $\begin{array}{l}\text { sample:solvent ratio } 1: 10 \\
\text { extraction temperature } 100{ }^{\circ} \mathrm{C} \\
\text { extraction time } 10 \mathrm{~min}\end{array}$ & $\begin{array}{l}\text { TPC, TAC, FRAP, ABTS, } \\
\text { delphinidin 3-sambubioside, } \\
\text { delphinidin 3-glucoside, } \\
\text { cyanidin 3-sambubioside, and } \\
\text { cyanidin 3-glucoside }\end{array}$ & [58] \\
\hline Mexico & maceration & $50 \%$ ethanol & $\begin{array}{l}\text { sample:solvent ratio } 1: 10 \\
\text { extraction time } 2 \mathrm{~h} \\
\text { light protection with } \\
\text { aluminum foil }\end{array}$ & $\begin{array}{c}\text { TPC, TAC, DPPH, } \\
\text { delphinidin-3-O- } \\
\text { sambubioside, } \\
\text { delphinidin-3-O-glucoside } \\
\text { (myrtillin), and } \\
\text { cyanidin-3-O-sambubioside) }\end{array}$ & [59] \\
\hline Vietnam & maceration & $50 \%$ ethanol & $\begin{array}{l}\text { sample:solvent ratio } 1: 8 \\
\text { extraction temperature } 60^{\circ} \mathrm{C} \\
\text { extraction time } 30 \mathrm{~min}\end{array}$ & TAC (180,82 mg Cy-3-GE/L) & [62] \\
\hline Brazil & maceration & $50 \%$ ethanol & $\begin{array}{l}\text { extraction temperature } 56^{\circ} \mathrm{C} \\
\text { pH } 10.2 \\
\text { extraction time } 110 \mathrm{~min}, \\
\text { stirring }\end{array}$ & TPC (5.01 mg GAE/g) & [63] \\
\hline Nigeria & maceration & methanol & $\begin{array}{l}\text { sample:solvent ratio 1:12.5 } \\
\text { extraction time } 72 \mathrm{~h}\end{array}$ & $\begin{array}{c}\text { Yield (27.3\%), TPC (29.2 mg } \\
\text { GAE/g) TFC (36.7 mg QE/g), } \\
\text { DPPH (78\%), and TBARS } \\
(21 \%)\end{array}$ & [65] \\
\hline Mexico & maceration & $96 \%$ ethanol & $\begin{array}{l}\text { sample:solvent ratio } 1: 50 \\
\text { extraction temperature } 65^{\circ} \mathrm{C}\end{array}$ & TAC (1.50 mg Cy-3-GE/g) & [78] \\
\hline Thailand & maceration & $95 \%$ ethanol & $\begin{array}{l}\text { sample:solvent ratio } 1: 4 \\
\text { extraction time } 24 \mathrm{~h} \\
\text { shaking at } 150 \mathrm{rpm}\end{array}$ & $\begin{array}{c}\text { Yield }(4.91 \%), \text { TPC }(0.05 \mathrm{mg} \\
\text { GAE } / \mathrm{g}), \mathrm{DPPH} \text {, gallic acid } \\
(0.75 \mathrm{~g} / \mathrm{kg}), \text { caffeic acid } \\
(0.39 \mathrm{~g} / \mathrm{kg}) \text {, ferulic acid } \\
(0.06 \mathrm{~g} / \mathrm{kg}), \text { chlorogenic acid } \\
(0.74 \mathrm{~g} / \mathrm{kg}) \text { and quercetin } \\
(0.45 \mathrm{~g} / \mathrm{kg})\end{array}$ & [81] \\
\hline Germany & maceration & $70 \%$ ethanol & $\begin{array}{l}\text { sample:solvent ratio } 1: 10 \\
\text { extraction temperature } 50{ }^{\circ} \mathrm{C} \\
\text { extraction time } 48 \mathrm{~h} \\
\text { shaking at } 150 \mathrm{rpm}\end{array}$ & $\begin{array}{c}\text { TPC }(0.04 \mu \mathrm{g} / \mathrm{mL}), \mathrm{TAC} \\
(3.16 \mathrm{mg} \mathrm{Cy}-3-\mathrm{GE} / \mathrm{L}), \text { and } \\
\text { DPPH }(60.38 \%)\end{array}$ & [82] \\
\hline Senegal & maceration & water & $\begin{array}{l}\text { sample:solvent ratio } 1: 15 \\
\text { extraction temperature } 30^{\circ} \mathrm{C} \\
\text { extraction time } 240 \mathrm{~min}\end{array}$ & $\begin{array}{c}\text { TAC (220 mg delphinidin } \\
\text { 3-xylosylglucoside/L), ORAC } \\
\text { (165 } \mu \text { mol Trolox/g) }\end{array}$ & [89] \\
\hline Iran & maceration & $\begin{array}{l}80 \% \text { ethanol } \\
\text { and water } \\
80 \% \text { methanol }\end{array}$ & $\begin{array}{l}\text { sample:solvent ratio } 1: 10 \\
\text { extraction time } 24 \mathrm{~h} \\
\text { sample:solvent ratio } 1: 10 \\
\text { extraction time } 24 \mathrm{~h}\end{array}$ & $\begin{array}{c}\text { TPC }(80 \% \text { ethanol: } 9.34 \mathrm{mg} / \mathrm{g} \text {; } \\
\text { water: } 9.59 \mathrm{mg} / \mathrm{g}), \mathrm{TFC}(80 \% \\
\text { ethanol: } 4.76 \mathrm{mg} / \mathrm{g} \text {; water: } \\
3.53 \mathrm{mg} / \mathrm{g}), \mathrm{TAC}(80 \% \\
\text { ethanol: } 0.042 \mathrm{mg} / \mathrm{g} \text {; water: } \\
0.044 \mathrm{mg} / \mathrm{g}) \\
\text { Caffeic acid }(5.08 \mathrm{mg} / \mathrm{g}), \\
\text { chlorogenic acid }(1.09 \mathrm{mg} / \mathrm{g}), \\
\text { p-coumaric acid }(0.07 \mathrm{mg} / \mathrm{g}), \\
\text { catechin }(0.92 \mathrm{mg} / \mathrm{g}), \\
\text { quercetin }(0.16 \mathrm{mg} / \mathrm{g}), \\
\text { hesperidin }(0.14 \mathrm{mg} / \mathrm{g}), \\
\text { hesperetin }(0.51 \mathrm{mg} / \mathrm{g})\end{array}$ & [34] \\
\hline
\end{tabular}


Table 1. Cont.

\begin{tabular}{|c|c|c|c|c|c|}
\hline $\begin{array}{l}\text { Country of } \\
\text { Origin }\end{array}$ & $\begin{array}{l}\text { Extraction } \\
\text { Method }\end{array}$ & Solvent & Extraction Condition & $\begin{array}{c}\text { Phenolics and Antioxidant } \\
\text { Activity }\end{array}$ & Ref \\
\hline Egypt & maceration & $50 \%$ ethanol & $\begin{array}{l}\text { sample:solvent ratio } 1: 50 \\
\text { extraction temperature } 180{ }^{\circ} \mathrm{C} \\
\text { extraction time } 30 \mathrm{~min}\end{array}$ & $\begin{array}{c}\text { TPC (43.1 mg GAE / g), gallic } \\
\text { acid }(0.91 \mathrm{mg} / \mathrm{g}), \\
\text { protocatechuic acid } \\
(163.20 \mathrm{mg} / \mathrm{g})\end{array}$ & [90] \\
\hline Mexico & maceration & distilled water & $\begin{array}{l}\text { sample:solvent ratio } 1: 50 \\
\text { extraction temperature } 95^{\circ} \mathrm{C} \\
\text { extraction time } 60 \mathrm{~min}\end{array}$ & $\begin{array}{c}\text { TPC, phenolic acids, } \\
\text { flavonoids, anthocyanins }\end{array}$ & [57] \\
\hline China & $\begin{array}{l}\text { maceration } \\
\text { UAE }\end{array}$ & $\begin{array}{l}0.1 \% \mathrm{HCl} \\
\text { aqueous } \\
\text { solution } \\
\mathrm{HP} \beta-\mathrm{CD} \\
\text { aqueous } \\
\text { solution }\end{array}$ & $\begin{array}{l}\text { sample:solvent ratio } 1: 15 \\
\text { extraction temperature } 25^{\circ} \mathrm{C} \\
\text { extraction time } 30 \mathrm{~min} \\
\text { sample:solvent ratio } 1: 27 \\
\text { extraction temperature } 54^{\circ} \mathrm{C} \\
\text { extraction time } 53 \mathrm{~min} \\
\quad 300 \mathrm{~W} ; 25 \mathrm{kHz}\end{array}$ & TAC $(4.80 \mathrm{mg} / \mathrm{g})$ & [33] \\
\hline Malaysia & UAE & $80 \%$ methanol & $\begin{array}{l}\text { sample:solvent ratio } 1: 20 \\
\text { extraction temperature } 60^{\circ} \mathrm{C} \\
\text { extraction time } 30 \mathrm{~min}\end{array}$ & $\begin{array}{c}\text { TPC, ACEI activity (0.01 } \\
\mu \mathrm{g} / \mathrm{mL}), 36 \text { identified } \\
\text { metabolites, including } \\
\text { flavonoids, anthocyanins, and } \\
\text { organic acids }\end{array}$ & {$[38]$} \\
\hline Brazil & UAE & $25 \%$ ethanol & $\begin{array}{c}\text { extraction temperature } 65^{\circ} \mathrm{C} \\
\text { extraction time } 45 \mathrm{~min}\end{array}$ & $\begin{array}{c}\text { TPC (2.24 mg GAE/g), TAC } \\
(3.58 \mathrm{mg} \mathrm{Cy-3-GE/g)}\end{array}$ & [9] \\
\hline Mexico & UAE & $80 \%$ ethanol & $\begin{array}{c}\text { sample:solvent ratio } 1: 20 \\
\text { extraction temperature } 60^{\circ} \mathrm{C} \\
\text { extraction time } 32 \mathrm{~min} \\
180 \mathrm{~W} ; 40 \mathrm{KHz}\end{array}$ & $\begin{array}{l}\text { Yield (red calyx: 20.84\%; } \\
\text { white calyx: } 13.74 \% \text { ), TPC (red } \\
\text { calyx: } 13.02 \mathrm{mg} \text { GAE/g; white } \\
\text { calyx: } 12.74 \mathrm{mg} \text { GAE/g), TFC } \\
\text { (red calyx: } 4.42 \mathrm{mg} \mathrm{CE/g;} \\
\text { white calyx: } 4.53 \mathrm{mg} \text { CE/g), } \\
\text { TAC (red calyx: } 1.80 \mathrm{mg} \\
\text { Cy-3-GE/g; white calyx: } \\
0.01 \mathrm{mg} \text { Cy-3-GE/g), DPPH } \\
\text { activity (red calyx: } 74.58 \% \text {; } \\
\text { white calyx: } 36.95 \% \text { ) }\end{array}$ & {$[10]$} \\
\hline Indonesia & UAE & water & $\begin{array}{l}\text { sample:solvent ratio } 1: 15 \\
\text { extraction at room } \\
\text { temperature } \\
\text { extraction time } 30 \mathrm{~min} \\
\text { frequency } 40 \mathrm{kHz}\end{array}$ & TAC & [54] \\
\hline Portugal & UAE & $39.1 \%$ ethanol & $\begin{array}{l}\text { extraction time } 26.1 \mathrm{~min} \\
\text { power } 296.6 \mathrm{~W}\end{array}$ & $\begin{array}{c}\text { TAC }(51.76 \mathrm{mg} / \mathrm{g}) \\
\text { delphinidin-3-O- } \\
\text { sambubioside, } \\
\text { cyanidin-3-O-sambubioside }\end{array}$ & [79] \\
\hline Malaysia & MAE & $52 \%$ ethanol & $\begin{array}{l}\text { sample:solvent ratio 1:15 } \\
\text { extraction time } 4 \mathrm{~min} \\
\text { microwave power } 450 \mathrm{~W}\end{array}$ & $\begin{array}{l}\text { TFC (94.32 mg QE/g), } \\
95 \text { flavonoid compounds }\end{array}$ & [32] \\
\hline NA & MAE & water & $\begin{array}{l}\text { sample:solvent ratio } 1: 10 \\
\text { extraction time } 15 \mathrm{~min} \\
\text { microwave power } 10 \mathrm{~W}\end{array}$ & yield $(24.6 \%)$, TPC $(2.72 \%)$ & [51] \\
\hline Turkey & MAE & $\begin{array}{l}\text { DES in } 50 \% \\
\text { water }\end{array}$ & microwave power $550 \mathrm{~W}$ & $\begin{array}{c}\text { TPC (31.90 mg GAE/g), TAC } \\
\text { (3.00 mg Cy-3-GE/g), DPPH } \\
95.89 \%\end{array}$ & [67] \\
\hline
\end{tabular}


Table 1. Cont.

\begin{tabular}{|c|c|c|c|c|c|}
\hline $\begin{array}{l}\text { Country of } \\
\text { Origin }\end{array}$ & $\begin{array}{l}\text { Extraction } \\
\text { Method }\end{array}$ & Solvent & Extraction Condition & $\begin{array}{c}\text { Phenolics and Antioxidant } \\
\text { Activity }\end{array}$ & Ref \\
\hline Malaysia & MAE & distilled water & $\begin{array}{l}\text { sample:solvent ratio } 1: 14 \\
\text { extraction temperature } 60^{\circ} \mathrm{C} \\
\text { extraction time } 3 \\
\text { minmicrowave power } 500 \mathrm{~W}\end{array}$ & $\begin{array}{l}\text { TPC (70.53 mg GAE/g) } \\
77 \text { phenolic compounds }\end{array}$ & [11] \\
\hline Spain & SFE & $16.7 \%$ ethanol & $\begin{array}{c}\text { extraction temperature } 64^{\circ} \mathrm{C} \\
\text { pressure } 391 \text { bar }\end{array}$ & $\begin{array}{c}\text { TPC (8.63 mg GAE/g) } \\
22 \text { identified and quantified } \\
\text { phenolic compounds }\end{array}$ & [12] \\
\hline Malaysia & SFE & $\begin{array}{c}\mathrm{CO}_{2} \\
\text { co-solvent } 75 \% \\
\text { ethanol }\end{array}$ & $\begin{array}{c}\text { extraction temperature } 70{ }^{\circ} \mathrm{C} \\
\text { extraction pressure } 8.90 \mathrm{MPa} \\
\text { flow rate } 9.49 \% \text { particle size } \\
350 \mu \mathrm{m}\end{array}$ & yield $26.73 \%$ & [85] \\
\hline Indonesia & SFE & $\begin{array}{c}\mathrm{CO}_{2} \\
\text { co-solvent } \\
\text { acetone }(5 \% \\
V / V)\end{array}$ & $\begin{array}{c}\text { extraction time } 2.5 \mathrm{~h} \\
\text { extraction temperature } 343.15 \\
\text { K pressure } 24 \mathrm{Mpa}\end{array}$ & TPC (8.63 mg GAE/g) & [80] \\
\hline
\end{tabular}

\subsection{Identification and Quantification of Phenolic Compounds}

The current studies have indicated that it is particularly important to accurately identify and quantify the levels of phenolics, especially in view of the complex chemistry of plant tissues, which contain a diverse range of primary and secondary metabolites. The determination of phenolic compounds remains challenging due to several constraints, such as numerous types of phenolic compounds with a wide range of polarities, different degrees of stability, the low levels of the compounds, and the matrix effect with interferences by impurities and compounds. Given the role of phenolic compounds as powerful antioxidants, the development of an optimal method for the extraction of multianalytes from a complex matrix presents some difficulties due to the possible rapid interactions of targeted analytes with other constituents in the matrix. Additionally, the determination of phenolic compounds is limited to the fact that these substances are structurally similar, thus possessing comparable absorption spectra in the UV wavelength range. A number of studies have already addressed this particular challenge of developing analytical methods for these compounds. Henceforth, this section aims to provide a specific review of the most updated analytical methods for determining phenolic compounds in roselle calyx, and Table 2 compiles the essential chromatography methods.

\subsubsection{Colorimetric Assays}

These assays were commonly applied to determine the phenolics, such as the total phenolics (TPC), total flavonoids (TFC), and total anthocyanins (TAC), from roselle calyx. The widely used approach for TPC is the Folin-Ciocalteu method $[3,34,52,58,59,64,65,68,75,76,81,82,90-93]$. The absorbance of the resulting complex of phenolic and Folin-Ciocalteu reagents, measured at $510 \mathrm{~nm}-700 \mathrm{~nm}$ by a spectrophotometer, indicates the level of TPC. The final calculation for the TPC is provided in units of mg gallic acid equivalents (GAE) per $g$ of fresh or dry calyces. In the TFC determination using the aluminum chloride method, the absorbance was measured at a wavelength of $415 \mathrm{~nm}-510 \mathrm{~nm}[34,52,64,65,68,75,76]$. Quercetin, rutin, and catechin are commonly used to establish the calibration curve for the TF quantification. Hence, the units to present the TFC level are mg quercetin equivalents (QE), rutin equivalents (RE), or catechin equivalents (CE) per $g$ of fresh or dry sample. In contrast to the TPC and TFC, the determination of the TAC can be performed without the prior establishment of a calibration curve using standard compounds. This approach measures the TAC level by a $\mathrm{pH}$ differential method employing $\mathrm{pH} 1$ and $4.5[6,34,78,94-96]$. The absorbance was measured at $510 \mathrm{~nm}-700 \mathrm{~nm}$. However, some proposed methods utilized external standards for the TAC quantification. 


\subsubsection{Liquid Chromatography}

Liquid chromatography has been the most-employed technique in roselle studies to measure phenolic compounds from roselle calyx, aiming to identify or quantify specific compounds. There is a growing interest in separation science to achieve still-better resolution, speed, and sensitivity in liquid chromatography. Significant advances in technology were made for ultra-fast separation with high efficiency through ultra-performance liquid chromatography (UPLC). UPLC has overcome the negative aspect of packed columns used in high-performance liquid chromatography (HPLC) by precisely delivering mobile phases at pressures up to 15,000 psi. The most used column type in roselle studies has been reverse phase $C_{18}[9,34,38,46,55,97]$, and the most widely used mobile phases have been acidified water and acetonitrile $[46,55,98,99]$ (Table 2). Reverse-phase $C_{18}$ is a hydrophobic column that is most suited for the separation of polar compounds of phenolics present in roselle. Additionally, acidified water and acetonitrile are commonly used for mobile phases as a result of the methods developed for phenolics determination by chromatographic techniques $[79,100]$. Almost all analyses were conducted using gradient elution with the analysis run time around 15-50 min. Various types of detectors, including UV-Vis, photodiode array detection (DAD), electrochemical detection (ECD), and fluorometric detection (FLD), are paired with the liquid chromatography instrument in accordance with the purpose of the analysis to improve the detection and quantification of the phenolic compounds. For phenolic compounds in roselle calyx, UV-Vis or DAD/PDA detectors are widely used to quantify specific compounds by comparing samples and the standard light absorption spectra (Table 2). Mass spectrophotometry (MS) detection is widely used in identification studies-identity based on the mass compound. Aiming to improve the method selectivity and sensitivity, several studies have combined two or more detectors $[28,55,79,99,101,102]$.

\subsubsection{Gas Chromatography (GC)}

GC instruments have been widely used in studies to determine the volatile phenolic compounds in roselle calyces (Table 2) [82,91,103-109]. The main constraints in GC application were derivatization procedures and the volatility of phenolic compounds [110]. This technique was designed as a powerful separation method of volatile compounds from a complex mixture using a heated column. The most widely used column to separate the volatile compounds of roselle has been a DB-Wax capillary column. The column was set with an initial temperature range from $40-50{ }^{\circ} \mathrm{C}$, then gradually increased to $200-250{ }^{\circ} \mathrm{C}$ to separate the compounds based on their volatility properties. Helium was the most frequently used carrier gas; however, some studies also proposed nitrogen and hydrogen carriers. The gas flow rate ranged from $1-1.7 \mathrm{~mL} / \mathrm{min}$. To identify and quantify the volatile compounds in roselle, the GC instruments were in tandem with MS, a flame ionization detector (FID), or coupled with olfactory detection.

\subsubsection{Thin-Layer Chromatography (TLC)}

An alternative for quick and cheap chromatography has also been developed-thinlayer chromatography (TLC). The studies employing the TLC method in roselle calyx are summarized in Table 2 . This analysis facilitates multiple compound detection on the same TLC plate during a single batch of analyses. The developed TLC method successfully separated glycosides, alkaloids, steroids, triterpenoids, tannins, and flavonoids based on their polarity in the roselle matrix. The separation procedure employed a mixture of chloroform and methanol (4:1) as a mobile phase and a thin plate coated with silica gel as a stationary phase. Subsequently, the TLC plate was exposed by a UV lamp at a wavelength of $254 \mathrm{~nm}$ [111]. Another study reported the TLC method for flavonoids from roselle tissue using butanol:acetic:water (4:1:5) and methanol:water (95:5) as the mobile phase, and the plates were observed at $265 \mathrm{~nm}$ [112]. 


\subsubsection{Miscellaneous Determination Techniques}

Emerging spectroscopy techniques have significantly improved the detection of phenolic compounds in roselle calyx. Apart from the aforementioned methods, the currently available techniques include near-infrared spectroscopy (NIR), nuclear magnetic resonance (NMR) spectroscopy, and fluorescence spectroscopy [27,39,71,113-115]. Nuclear magnetic resonance (NMR) spectroscopy was employed in the isolation of protocatechuic acid, gallic acid, and anthocyanin derivatives [71,113]. A combination of NMR and RP-UHPLCMS [39] or HPLC-ESI/MS [71] was able to identify phenolics in the purified extracts of roselle. UHPLC-MS offered a higher detection level and a more reliable model than NMR, although NMR proved to be more potent in the structural characterization and precise quantification of metabolites [39]. The use of low-field NMR (LF-NMR) with NIR and fluorescence spectroscopy was reported in a study of geographical origin authentication of roselle. The study was conducted by determining compounds in 64 roselle samples from Sudan and eight from China. The resulting data were processed with a principal components analysis (PCA), hierarchical cluster analysis (HCA), and PCA combined with linear discriminant analysis (PCA-LDA). The proposed method combined with chemometrics was feasible for grouping roselle samples by country of origin [27].

Table 2. Recent chromatography methods to determine the phenolic compounds in roselle calyx. Abbreviations: N/A: not available, LC: liquid chromatography, HPLC: high-performance liquid chromatography, UPLC: ultra-performance liquid chromatography, GC: gas chromatography, TLC: thin-layer chromatography, UV-Vis: ultraviolet-visible, DAD: diode array detector, PDA: photodiode array detector, FID: flame ionization detector, MS: mass spectrophotometry, O: olfactometry, ESI: electrospray ionization, TOF/QTOF: quadrupole time-of-flight, and NMR: nuclear magnetic resonance.

\begin{tabular}{|c|c|c|c|c|}
\hline Pre-Analysis Step & $\begin{array}{c}\text { Determination } \\
\text { Method }\end{array}$ & Analysis Condition & Target Compound & Ref \\
\hline $\begin{array}{l}\text { Solvent evaporation } \\
\text { at } 35^{\circ} \mathrm{C} \text { and the } \\
\text { obtained residue } \\
\text { redissolved in } \\
\text { water }\end{array}$ & LC-DAD-ESI/MS & $\begin{array}{c}\text { Column: Waters C18 } \\
(4.6 \mathrm{~mm} \times 150 \mathrm{~mm}, 3 \mu \mathrm{m}) \\
\text { Mobile phase: }(\mathrm{A}) 0.1 \% \text { trifluoroacetic } \\
\text { acid in water }(\mathrm{B}) \text { acetonitrile } \\
\text { Elution: gradient }\end{array}$ & $\begin{array}{l}\text { Delphinidin-3-O-sambubioside } \\
\text { and cyanidin-3-O-sambubioside }\end{array}$ & [79] \\
\hline $\mathrm{N} / \mathrm{A}$ & $\begin{array}{l}\text { LC-DAD-MS } \\
\text { HPLC-PDA }\end{array}$ & $\begin{array}{c}\text { Column: Dionex C18 } \\
(250 \mathrm{~mm} \times 4.6 \mathrm{~mm}, 5 \mu \mathrm{m}) \\
\text { Mobile phase: }(\mathrm{A}) \text { water (B) } 60 \% \\
\text { methanol in water } \\
\text { Elution: gradient } \\
\text { Column: Dionex } \mathrm{C} 18 \\
(250 \mathrm{~mm} \times 4.6 \mathrm{~mm}, 5 \mu \mathrm{m}) \\
\text { Mobile phase: }(\mathrm{A}) \text { water (B) } 60 \% \\
\text { methanol in water } \\
\text { Elution: gradient }\end{array}$ & $\begin{array}{l}\text { Chlorogenic acid, gallic acid, } \\
\text { protocatechuic acid, quercetin, } \\
\text { delphinidin-3-glucoside, and } \\
\text { cyanidin-3-glucoside }\end{array}$ & [83] \\
\hline $\mathrm{N} / \mathrm{A}$ & LC-ESI-MS/MS & $\begin{array}{c}\text { Column: C18 } \\
(100 \mathrm{~mm} \times 2.1 \mathrm{~mm} \times 1.8 \mu \mathrm{m}, 5 \mu \mathrm{m}) \\
\text { Elution: gradient }\end{array}$ & 77 phenolic compounds & [11] \\
\hline $\mathrm{N} / \mathrm{A}$ & $\begin{array}{l}\text { LC-MS- } \\
\text { TOFHPLC-DAD }\end{array}$ & $\begin{array}{l}\text { Column: Phenomenex Gemini C18 } \\
(250 \mathrm{~mm} \times 4.6 \mathrm{~mm}, 5 \mu \mathrm{m}) . \\
\text { Mobile phase: }(\mathrm{A}) 0.1 \%(v / v) \\
\text { trifluoroacetic acid (B) trifluoroacetic } \\
\text { acid/acetonitrile/water }(50: 49.9: 0.1) \\
\text { Flow rate: } 1 \mathrm{~mL} / \mathrm{min} \\
\text { Elution: gradient }\end{array}$ & $\begin{array}{c}\text { Gallic acid, protocatechuic acid, } \\
\text { 3-O-caffeoylquinic acid caffeic } \\
\text { acid myricetin } \\
\text { 3-O-arabinogalactoside } \\
\text { quercetin 3-O-sambubioside } \\
\text { delphinidin 3-O-sambubioside } \\
\text { delphinidin 3-O-glucoside } \\
\text { cyanidin 3-O-sambubioside }\end{array}$ & [30] \\
\hline
\end{tabular}


Table 2. Cont.

\begin{tabular}{|c|c|c|c|c|}
\hline Pre-Analysis Step & $\begin{array}{l}\text { Determination } \\
\text { Method }\end{array}$ & Analysis Condition & Target Compound & Ref \\
\hline $\mathrm{N} / \mathrm{A}$ & $\begin{array}{l}\text { HPLC-PDA } \\
\text { HPLC- } \\
\text { Q/TOF/MS/ESI }\end{array}$ & $\begin{array}{c}\text { Column: Gemini C18 } \\
(250 \mathrm{~mm} \times 4.6 \mathrm{~mm}, 5 \mu \mathrm{m}) \\
\text { Mobile phase: }(\mathrm{A}) 0.1 \%(v / v) \\
\text { trifluoroacetic acid (B) trifluoroacetic } \\
\text { acid/acetonitrile/water }(50: 49.9: 0.1) \\
\text { Elution: gradient } \\
\text { Column: Kinetex } \mathrm{C} 18 \\
(4.6 \mathrm{~mm} \times 150 \mathrm{~mm}, 2.6 \mu \mathrm{m}) \\
\text { Mobile phase: }(\mathrm{A}) 0.5 \%(v / v) \text { formic } \\
\text { acid in water }(\mathrm{B}) 0.5 \%(v / v) \text { formic } \\
\text { acid in acetonitrile } \\
\text { Elution: gradient }\end{array}$ & $\begin{array}{l}\text { Quantification: gallic acid, } \\
\text { caffeic acid, delphinidin } \\
\text { 3-O-sambubioside, cyanidin } \\
\text { 3-O-sambubioside, } \\
\text { myricetin-3-arabinogalactoside, } \\
\text { quercetin-3-sambubioside } \\
\text { Identification: } 11 \text { derivatives } \\
\text { anthocyanins and } \\
\text { pyranoanthocyanins }\end{array}$ & [109] \\
\hline $\mathrm{N} / \mathrm{A}$ & HPLC & $\begin{array}{c}\text { Column: C18 column } \\
(250 \mathrm{~mm} \times 4.6 \mu \mathrm{m}, 5 \mu \mathrm{m}) \\
\text { Mobile phase: }(\mathrm{A}) 100 \% \text { methanol }(\mathrm{B}) \\
0.1 \% \text { trifluoroacetic acid } \\
\text { Elution: gradient }\end{array}$ & $\begin{array}{l}\text { Gallic acid, protocatechuic acid, } \\
\text { p-hydroxybenzoic, chlorogenic } \\
\text { acid, caffeic acid, syringic acid, } \\
p \text {-coumaric acid, and ferulic acid }\end{array}$ & [92] \\
\hline $\mathrm{N} / \mathrm{A}$ & HPLC-UV & $\begin{array}{c}\text { Column: RP }(150 \mathrm{~mm} \times 4.6 \mathrm{~mm}, 3 \mu \mathrm{m}) \\
\text { Mobile phase: }(\mathrm{A}) 1 \% \text { acetic acid in } \\
\text { acetonitrile (B) } 1 \% \text { acetic acid in water } \\
\text { Elution: gradient }\end{array}$ & $\begin{array}{l}\text { Gallic acid and protocatechuic } \\
\text { acid }\end{array}$ & [90] \\
\hline $\mathrm{N} / \mathrm{A}$ & HPLC-UV-Vis & $\begin{array}{c}\text { Column: Supelco Discovery HS C18 } \\
(250 \mathrm{~mm} \times 4.6 \mathrm{~mm}, 5 \mu \mathrm{m}) \\
\text { Mobile phase: }(\mathrm{A}) 10 \% \text { acetonitrile (B) } \\
90 \% \text { formic acid }(2 \%) \\
\text { Elution: gradient }\end{array}$ & $\begin{array}{l}\text { Delphinidin-3-sambubioside, } \\
\text { cyanidin-3-sambubioside, rutin, } \\
\text { chlorogenic acid }\end{array}$ & [86] \\
\hline $\begin{array}{l}\text { The extract was } \\
\text { diluted in methanol }\end{array}$ & HPLC-UV-Vis & $\begin{array}{c}\text { Column: RP-Phenomenex C18 } \\
(250 \mathrm{~mm} \times 4.6 \mathrm{~mm}, 5 \mu \mathrm{m}) \\
\text { Mobile phase: }(\mathrm{A}) \text { formic acid-water } \\
\text { 1:99 } v / v(\mathrm{~B}) \text { acetonitrile } \\
\text { Elution: gradient }\end{array}$ & $\begin{array}{l}\text { Phenolic acids, flavonoids, } \\
\text { anthocyanins }\end{array}$ & [57] \\
\hline $\mathrm{N} / \mathrm{A}$ & $\begin{array}{l}\text { HPLC-UV } \\
\text { ESI-TOF-MS }\end{array}$ & $\begin{array}{l}\text { Column: Zorbax Eclipse Plus C18 } \\
\text { (150 mm } \times 4.6 \mathrm{~mm}, 1.8 \mu \mathrm{m}) \\
\text { Flow rate: } 0.5 \mathrm{~mL} / \mathrm{min} \\
\text { Injection volume: } 10 \mu \mathrm{L} \\
\text { Mobile phase for non-anthocyanin } \\
\text { compounds: (A) acidified }(1 \% \text { acetic } \\
\text { acid) water:acetonitrile } 90: 10 \text { (B) } \\
\text { acetonitrile } \\
\text { Elution: gradient } \\
\text { Mobile phase for anthocyanin } \\
\text { compounds: (A) acidified water } \\
\text { (10\% acetic acid) and (B) acetonitrile } \\
\text { Elution: gradient } \\
\text { Instrument: microTOF, ESI-TOF mass } \\
\text { spectrometer }\end{array}$ & $\begin{array}{l}\text { Chlorogenic acid, quercetin } \\
\text { 3-rutinoside, quercetin } \\
\text { 3-glucoside, kaempferol } \\
\text { 3-O-rutinoside and kaempferol } \\
\text { 3-( } p \text {-coumarylglucoside), } \\
\text { quercetin, 4-hydroxycoumarin } \\
\text { and delphinidin-3-sambubioside }\end{array}$ & [116] \\
\hline $\begin{array}{l}\text { Dried extract was } \\
\text { redissolved in } 4 \% \\
\text { acetic acid in water }\end{array}$ & HPLC-DAD & $\begin{array}{c}\text { Column: C18 column } \\
\left(300 \mathrm{~mm} \times 3.9 \mathrm{~mm}, 125 \mathrm{~A}^{\circ}\right) \\
\text { Mobile phase: }(\mathrm{A}) 4 \% \text { acetic acid in } \\
\text { water (B) } 100 \% \text { acetonitrile } \\
\text { Elution: gradient }\end{array}$ & Cyanidin 3-sambubioside & [117] \\
\hline
\end{tabular}


Table 2. Cont.

\begin{tabular}{|c|c|c|c|c|}
\hline Pre-Analysis Step & $\begin{array}{c}\text { Determination } \\
\text { Method }\end{array}$ & Analysis Condition & Target Compound & Ref \\
\hline $\mathrm{N} / \mathrm{A}$ & HPLC -DAD & $\begin{array}{c}\text { Column: RP C18 } \\
(25 \mathrm{~cm} \times 0.4 \mathrm{~cm}, 5 \mathrm{~mm}) \\
\text { Mobile phase: acetonitrile:formic } \\
\text { acid }(4.5 \%) \\
\text { Elution: isocratic }\end{array}$ & $\begin{array}{c}\text { Anthocyanins } \\
\text { (delphinidin-3-O-sambubioside, } \\
\text { delphinidin-3-O-glucoside } \\
\text { (myrtillin), and } \\
\text { cyanidin-3-O-sambubioside) }\end{array}$ & [59] \\
\hline $\mathrm{N} / \mathrm{A}$ & HPLC -DAD & $\begin{array}{c}\text { Column: C18 }(250 \mathrm{~mm} \times 4.6 \mathrm{~mm}) \\
\text { Mobile phase: }(\mathrm{A}) \\
\text { water:acetonitrile:trifluoroacetic acid } \\
\text { (50:50:0.1) and (B) } \\
\text { acetonitrile:water:trifluoroacetic acid } \\
\text { (10:90:0.1) } \\
\text { Elution: gradient }\end{array}$ & $\begin{array}{l}\text { Gallic acid, protocatechuic acid, } \\
\text { caffeic acid, gentisic, chlorogenic } \\
\text { acid, vanillic acid, benzoic acid, } \\
\text { syringic acid, catechin, and } \\
\text { epicatechin }\end{array}$ & [35] \\
\hline $\begin{array}{l}80 \% \text { methanol } \\
\text { extract }\end{array}$ & HPLC-DAD & $\begin{array}{c}\text { Column: zorbax eclipse XDB-C18 } \\
\text { column }(4.6 \mathrm{~mm} \times 150 \mathrm{~mm}, 5 \mu \mathrm{m}) \\
\text { Mobile phase: }(\mathrm{A}) \text { methanol } 100 \%(\mathrm{~B}) \\
\text { formic acid } 1 \% ; \\
\text { Elution: gradient }\end{array}$ & $\begin{array}{c}\text { Caffeic acid, chlorogenic acid, } \\
\text { p-coumaric acid, catechin, } \\
\text { quercetin, hesperidin, hesperetin }\end{array}$ & [34] \\
\hline $\begin{array}{l}\text { Extract was } \\
\text { re-dissolved in } 2.5 \\
\text { mL methanol }\end{array}$ & HPLC-DAD & $\begin{array}{l}\text { Column: C18 reversed-phase Xbridge } \\
(250 \mathrm{~mm} \times 4.6 \mathrm{~mm}, 5 \mu \mathrm{m}) \text { Mobile } \\
\text { phase: (A) } 0.1 \% \text { acetic acid in distilled } \\
\text { water (B) acetonitrile } \\
\text { Injection volume: } 10 \mu \mathrm{L} \\
\text { Flow rate: } 0.8 \mathrm{~mL} / \mathrm{min}\end{array}$ & Protocatechuic acid, catechin & [46] \\
\hline $\mathrm{N} / \mathrm{A}$ & HPLC-DAD & $\begin{array}{c}\text { Column: C18 } \\
(150 \mathrm{~mm} \times 4.6 \mathrm{~mm}, 5 \mu \mathrm{m}) \\
\text { Mobile phase: }(\mathrm{A}) 2 \% \text { acetic acid (B) } \\
100 \% \text { methanol } \\
\text { Elution: gradient }\end{array}$ & $\begin{array}{l}\text { Gallic acid, chlorogenic acid, } \\
\text { caffeic acid, ferulic acid, catechin, } \\
\text { epicatechin, rutin, quercetin, } \\
\text { quercitrin, and kaempferol }\end{array}$ & [97] \\
\hline $\mathrm{N} / \mathrm{A}$ & HPLC-DAD & $\begin{array}{c}\text { Column: Zorbax C18 } \\
(250 \mathrm{~mm} \times 4.6 \mathrm{~mm}, 5 \mu \mathrm{m}) \\
\text { Mobile phase: }(\mathrm{A}) 0.1 \% \text { trifluoroacetic } \\
\text { acid (B) acetonitrile } \\
\text { Elution: gradient }\end{array}$ & $\begin{array}{l}\text { Cyanidin 3-sambubioside } \\
\text { (Cy-3-Sa), } \\
\text { delphinidin-3-sambubioside }\end{array}$ & [73] \\
\hline $\mathrm{N} / \mathrm{A}$ & HPLC-DAD & $\begin{array}{c}\text { Column: } \mathrm{C} 18(250 \mathrm{~mm} \times 4 \mathrm{~mm}, 5 \mu \mathrm{m}) \\
\text { Mobile phase: }(\mathrm{A}) \\
\text { water/acetonitrile/formic acid } \\
(87 \mathrm{I}: 3 \mathrm{I}: 10 \% v / v / v)(\mathrm{B}) \\
\text { water / acetonitrile/formic acid } \\
\text { (40I:50I:10\% } v / v / v) \\
\text { Elution: gradient }\end{array}$ & $\begin{array}{l}\text { delphinidin chloride, malvidin } \\
\text { chloride, cyanidin chloride and } \\
\text { pelargonidin chloride }\end{array}$ & {$[70]$} \\
\hline $\mathrm{N} / \mathrm{A}$ & HPLC-DAD & $\begin{array}{c}\text { Non-anthocyanins } \\
\text { column: C18 Poroshell } \\
(50 \mathrm{~mm} \times 4 \mathrm{~mm} \times 6 \mathrm{~mm}, 2.7 \mu \mathrm{m}) \\
\text { Mobile phase: }(\mathrm{A}) 0.1 \% \text { formic acid in } \\
\text { water (B) acetonitrile } \\
\text { Anthocyanins column: Zorbax } \mathrm{C} 18 \\
\text { column }(250 \mathrm{~mm} \times 4.6 \mathrm{~mm}, 5 \mu \mathrm{m}) \\
\text { Mobile phase: }(\mathrm{A}) \text { trifluoroacetic acid, } \\
0.1 \%)(\mathrm{B}) \text { acetonitrile } \\
\text { Elution: gradient }\end{array}$ & $\begin{array}{l}\text { Flavanols, flavonols, benzoic, } \\
\text { hibiscus and phenolic acids as } \\
\text { well as two main anthocyanins } \\
\text { (cyanidin 3sambubioside and } \\
\text { delphinidin 3-sambubioside) }\end{array}$ & [98] \\
\hline
\end{tabular}


Table 2. Cont.

\begin{tabular}{|c|c|c|c|c|}
\hline Pre-Analysis Step & $\begin{array}{l}\text { Determination } \\
\text { Method }\end{array}$ & Analysis Condition & Target Compound & Ref \\
\hline $\mathrm{N} / \mathrm{A}$ & HPLC-DAD & $\begin{array}{l}\text { Column: hypersil ODS.2 analytical } \\
(250 \mathrm{~mm} \times 46 \mathrm{~mm}, 5 \mu \mathrm{m}) \\
\text { Mobile phase: methanol:acetic } \\
\text { acid:water }(25: 1: 75 \mathrm{v} / \mathrm{v} / \mathrm{v}) \\
\text { Elution: isocratic }\end{array}$ & $\begin{array}{l}\text { Caffeic acid, chlorogenic acid, } \\
\text { ferulic acid, gallic acid, } \\
\text { protocatechuic acid, } p \text {-coumaric } \\
\text { acid, p-hydroxybenzoic acid and } \\
\text { syringic acid }\end{array}$ & [118] \\
\hline $\mathrm{N} / \mathrm{A}$ & HPLC-DAD & $\begin{array}{c}\text { Column: RP Alltech Prevail C18 } \\
(250 \mathrm{~mm} \times 4.6 \mathrm{~mm}, 5 \mu \mathrm{m}) \\
\text { Mobile phase: }(\mathrm{A}) \mathrm{AcCN}(\mathrm{B}) 3 \% \text { aq. } \\
\text { AcOH } \\
\text { Elution: gradient }\end{array}$ & $\begin{array}{c}\text { Gallic acid, caffeic acid, ferulic } \\
\text { acid, chlorogenic acid and } \\
\text { quercetin }\end{array}$ & [81] \\
\hline $\mathrm{N} / \mathrm{A}$ & HPLC-DAD & $\begin{array}{c}\text { Column: Kromasil C18 } \\
(250 \mathrm{~mm} \times 4.6 \mathrm{~mm}, 5 \mu \mathrm{m}) \\
\text { Mobile phase: }(\mathrm{A}) 10 \mathrm{mM} \text { phosphoric } \\
\text { acid (pH } 2.5)(\mathrm{B}) 100 \% \text { methanol } \\
\text { Elution: gradient }\end{array}$ & Caffeic acid and $p$-coumaric acid & [114] \\
\hline $\mathrm{N} / \mathrm{A}$ & HPLC-DAD & $\begin{array}{c}\text { Column: ACE®C18 } \\
(250 \mathrm{~mm} \times 4.6 \mathrm{~mm}, 5 \mu \mathrm{m}) \\
\text { Mobile phase: }(\mathrm{A}) \text { acetonitrile }(\mathrm{B}) 0.1 \% \\
\text { trifluoroacetic acid (TFA) } \\
\text { Elution: gradient }\end{array}$ & $\begin{array}{l}\text { Anthocyanins (cyanidin } \\
\text { 3-glucoside, delphinidin } \\
\text { 3-glucoside, malvidin } \\
\text { 3-glucoside, and peonidin } \\
\text { 3-glucoside) and anthocyanidins } \\
\text { (Cyanidin chloride, delphinidin } \\
\text { chloride, malvidin chloride, and } \\
\text { peonidin chloride) }\end{array}$ & [119] \\
\hline $\mathrm{N} / \mathrm{A}$ & HPLC-DAD & $\begin{array}{c}\text { Column: RP- ACEC18 } \\
(250 \mathrm{~mm} \times 4.6 \mathrm{~mm}, 5 \mu \mathrm{m}) \\
\text { Mobile phase: }(\mathrm{A}) \text { water } / \text { formic } \\
\text { acid/acetonitrile }(97.2 / 2.0 / 0.8 \mathrm{v} / \mathrm{v} / \mathrm{v}) \\
\text { (B) acetonitrile } \\
\text { Elution: gradient }\end{array}$ & $\begin{array}{c}\text { Anthocyanins (Del 3-O-Sb and } \\
\text { Cya 3-O-Sb), gallic and } \\
\text { protocatechuic acids }\end{array}$ & [120] \\
\hline $\begin{array}{c}\mathrm{N} / \mathrm{A} \\
\text { Extract in methanol }\end{array}$ & $\begin{array}{l}\text { HPLC-DAD } \\
\text { HPLC-ESI-MS }\end{array}$ & $\begin{array}{c}\text { Column: promosil C18 } \\
(250 \mathrm{~mm} \times 4.6 \mathrm{~mm}, 5 \mu \mathrm{m}) \text {; Mobile } \\
\text { phase: }(\mathrm{A}) 1.5 \% \text { formic acid aqueous } \\
\text { solution (B) acetonitrile } \\
\text { Column: promosil C18 } \\
(250 \mathrm{~mm} \times 4.6 \mathrm{~mm}, 5 \mu \mathrm{m}) \text { Mobile } \\
\text { phase: (A) } 0.1 \% \text { formic acid aqueous } \\
\text { solution (B) methanol containing } 0.1 \% \\
\text { formic acid } \\
\text { Elution: gradient }\end{array}$ & $\begin{array}{l}\text { Delphinidin-3-Osambubioside } \\
\text { (D3S) }\end{array}$ & [87] \\
\hline $\mathrm{N} / \mathrm{A}$ & $\begin{array}{l}\text { HPLC-DADLC- } \\
\text { MS }\end{array}$ & $\begin{array}{c}\text { Column: Gemini C18 } \\
(250 \mathrm{~mm} \times 4.6 \mathrm{~mm}, 5 \mu \mathrm{m}) \\
\text { Mobile phase: }(\mathrm{A}) 0.2 \%(v / v) \text { formic } \\
\text { acid in water, (B) acetonitrile } \\
\text { Elution: gradient }\end{array}$ & $\begin{array}{l}\text { Delphinidin 3-sambubioside, } \\
\text { delphinidin 3-glucoside, } \\
\text { cyanidin 3-sambubioside and } \\
\text { cyanidin 3-glucoside }\end{array}$ & [58] \\
\hline
\end{tabular}


Table 2. Cont.

\begin{tabular}{|c|c|c|c|c|}
\hline Pre-Analysis Step & $\begin{array}{l}\text { Determination } \\
\text { Method }\end{array}$ & Analysis Condition & Target Compound & Ref \\
\hline $\begin{array}{l}\text { Purification using } \\
\text { TLC }\end{array}$ & $\begin{array}{l}\text { HPLC-DAD } \\
\text { HPLC-ESI-MS }\end{array}$ & $\begin{array}{c}\text { Column: Kinetex C18 } \\
(250 \mathrm{~mm} \times 4.6 \mathrm{~mm}, 5 \mu \mathrm{m}) \\
\text { Mobile phase: }(\mathrm{A}) \text { formic acid } \\
(5 \%, v / v)(\mathrm{B}) \text { acetonitrile } \\
(5 \%, v / v \text { formic acid }) \\
\text { Elution: gradient } \\
\text { Column: A DIONEX Acclaim } 120 \mathrm{C} 18 \\
(150 \mathrm{~mm} \times 2.1 \mathrm{~mm}, 3 \mu \mathrm{m}) \\
\text { Mobile phase: }(\mathrm{A}) \text { formic acid } \\
(5 \%, v / v)(\mathrm{B}) \text { acetonitrile } \\
(5 \% \text { formic acid, } v / v) \\
\text { Elution: gradient }\end{array}$ & Anthocyanins & [88] \\
\hline $\begin{array}{c}\text { Diluted with } \\
\text { purified distilled } \\
\text { water }\end{array}$ & $\begin{array}{l}\text { HPLC-DAD } \\
\text { NMR }\end{array}$ & $\begin{array}{c}\text { Column: prontosil C-18 } \\
(250 \mathrm{~mm} \times 4 \mathrm{~mm}, 5 \mu \mathrm{m}) \\
\text { Mobile phase: (A) } 0.1 \% \text { trifluoroacetic } \\
\text { acid in water (B) } 0.1 \% \text { trifluoroacetic } \\
\text { acid in acetonitrile } \\
\text { Elution: gradient }\end{array}$ & $\begin{array}{l}\text { Protocatechuic acid and } \\
\text { anthocyanins }\end{array}$ & [121] \\
\hline $\begin{array}{l}\text { Diluted with } \\
\text { ultrapure water }\end{array}$ & $\begin{array}{l}\text { HPLC-DAD-ESI- } \\
\text { TOF }\end{array}$ & $\begin{array}{c}\text { Column: Zorbax C18 } \\
(250 \mathrm{~mm} \times 4.6 \mathrm{~mm}, 5 \mu \mathrm{m}) \\
\text { Mobile phase: } 50: 49.99: 0.01(\mathrm{v} / \mathrm{v}) \\
\text { methanol:water:trifluoroacetic acid } \\
\text { Elution: isocratic }\end{array}$ & Anthocyanins & [122] \\
\hline $\begin{array}{l}\text { Diluted with } \\
\text { solvent A }\end{array}$ & $\begin{array}{c}\text { HPLC-DAD-ESI- } \\
\text { MS/MS }\end{array}$ & $\begin{array}{c}\text { Column: } \mathrm{C} 18 \\
(150 \mathrm{~mm} \times 4.6 \mathrm{~mm}, 4.0 \mu \mathrm{m}) \\
\text { Mobile phase: }(\mathrm{A}) \text { water } / \text { formic acid } \\
(99.5: 0.5, v / v)(\mathrm{B}) \text { acetonitrile } / \text { formic } \\
\text { acid }(99.5: 0.5, v / v) \\
\text { Elution: gradient }\end{array}$ & $\begin{array}{c}\text { 3-caffeoylquinic acid, } \\
\text { delphinidin 3-sambubioside, } \\
\text { 3-p-coumaroylquinic acid, } \\
\text { cyanidin 3-sambubioside, } \\
\text { 5-caffeoylquinic acid, } \\
\text { 4-caffeoylquinic acid, myricetin } \\
\text { 3-sambubioside, quercetin } \\
\text { 3-sambubioside, } \\
\text { 5-O-caffeoylshikimic acid, } \\
\text { 5- } \text {-coumaroylquinic acid, } \\
\text { quercetin 3-rutinoside, quercetin } \\
\text { 3-glucoside, kaempferol } \\
\text { 3-O-rutinoside }\end{array}$ & [8] \\
\hline
\end{tabular}

Column: phenomenex $\mathrm{C} 18$

Concentrated and resuspended in the mobile phase
HPLC-DAD-MS
Mobile phase: (A) water: acetonitrile:

formic acid 90:10:1 $(v / v)(B)$

acetonitrile $(v / v)$

Elution: gradient
Anthocyanins
Identification column: ACEC18

$(250 \mathrm{~mm} \times 4.6 \mathrm{~mm}, 5 \mu \mathrm{m})$

Mobile phase: (A) water/formic acid/acetonitrile $(99.1 / 0.1 / 0.8 v / v / v)$

(B) acetonitrile

Injection volume: $20 \mu \mathrm{L}$

Flow rate: $0.7 \mathrm{~mL} \mathrm{~min}$

Elution: gradient.

Quantification: the same operating conditions as for identification with Mobile phase: (A) 97.2/2/0.8 (v/v/v)

of water/formic acid/acetonitrile.
Anthocyanins, flavonols, chlorogenic acid, gallic acid, and protocatechuic acid 
Table 2. Cont.

\begin{tabular}{|c|c|c|c|c|}
\hline Pre-Analysis Step & $\begin{array}{l}\text { Determination } \\
\text { Method }\end{array}$ & Analysis Condition & Target Compound & Ref \\
\hline $\mathrm{N} / \mathrm{A}$ & $\begin{array}{l}\text { HPLC-DAD- } \\
\text { MS/MS }\end{array}$ & $\begin{array}{l}\text { Column: Synergi Hydro-RP C18 } \\
(250 \mathrm{~mm} \times 4.6 \mathrm{~mm}, 4 \mu \mathrm{m}) \\
\text { Mobile phase: }(\mathrm{A}) \text { water-formic acid } \\
(99.5: 0.5, v / v)(\mathrm{B}) \text { acetonitrile-formic } \\
\text { acid }(99.5: 0.5, v / v) \\
\text { Elution: gradient }\end{array}$ & $\begin{array}{l}20 \text { identified and quantified } \\
\text { phenolic compounds. Major } \\
\text { found as } \\
\text { delphinidin-3-O-sambubioside } \\
\text { and 3-caffeoylquinic acid }\end{array}$ & [102] \\
\hline $\mathrm{N} / \mathrm{A}$ & HPLC-PDA & $\begin{array}{l}\text { Column: C18 Phenomenax Luna } \\
(150 \mathrm{~mm} \times 4.6 \mathrm{~mm}, 10 \mu \mathrm{m}) \\
\text { Mobile phase: }(\mathrm{A}) 0.1 \% \text { phosphoric } \\
\text { acid (B) } 100 \% \text { acetonitrile } \\
\text { Elution: gradient }\end{array}$ & $\begin{array}{l}\text { Chlorogenic acid, coumaric acid, } \\
\text { ferulic acid, quercetin and } \\
\text { cyanidine-3-sambubioside }\end{array}$ & [3] \\
\hline
\end{tabular}

Dissolved in $1 \mathrm{~mL}$ of $0.1 \%$ formic acid in water, sonicated for $5 \mathrm{~min}$

HPLC-PDA

Column: Purospher STAR RP-18e LichroCART

$(250 \mathrm{~mm} \times 4.6 \mathrm{~mm}, 5 \mu \mathrm{m})$

Mobile phase: (A) $0.1 \%$ formic acid in water (B) $0.1 \%$ formic acid in acetonitrile

Elution: gradient

Column: Purospher STAR RP-18e

LichroCART column

$(250 \mathrm{~mm} \times 4.6 \mathrm{~mm}, 5 \mu \mathrm{m})$

Dissolved in $1 \mathrm{~mL}$ in water. Samples were sonicated for 5

HPLC-PDA $\min$

HPLC-PDA

HPLC-ESI-

Purification and

MSNMR

fractionation

HPLC-ESI-TOFMS
$\mathrm{N} / \mathrm{A}$
HPLC-ESI-TOFMS
Mobile phase: (A) $0.1 \%$ formic acid in water (B) $0.1 \%$ formic acid in acetonitrile Elution: gradient
Column: RP C18 (3.0 mm, $5 \mu \mathrm{m})$

Mobile phase: (A) $0.1 \%$ trifluoroacetic acid (B) acetonitrile $(85, v / v$, $0.085 \%)$ Elution: gradient

Column: RP-18 Lichrocart $v / v)(\mathrm{B})(\mathrm{MeCN}($ formic acid $1 \%, v / v))$

Ascorbic acid, chlorogenic acid, caffeic acid,

delphinidin-3-O-sambubioside and cyanidin-3-O-sambubioside

Gallic acid, ascorbic acid, chlorogenic acid, caffeic acid, delphinidin-3-O-sambubioside, and cyanidin-3-O-sambubioside

(+)catechin, gallic, chlorogenic, caffeic, syringic and ferulic acids $(150 \mathrm{~mm} \times 4.6 \mathrm{~mm}, 5 \mu \mathrm{m})$ Mobile phase: (A) [formic acid (1\%, Elution: gradient

Column: Zorbax Eclipse Plus C18 $(150 \mathrm{~mm} \times 4.6 \mathrm{~mm}, 1.8 \mu \mathrm{m})$

Mobile phase: (A) water plus $0.1 \%$ of formic acid (B) acetonitrile Elution: gradient

delphinidin-3-O-sambubioside (Dp-samb) and

cyanidin-3-O-sambubioside

22 identified and quantified phenolic compounds, including: organic acid, phenolic acid, and

Column: Zorbax Eclipse Plus C18 $(150 \mathrm{~mm} \times 4.6 \mathrm{~mm}, 1.8 \mu \mathrm{m})$

Mobile phase: $(\mathrm{A})$ water plus $0.1 \%$ of formic acid (B) acetonitrile Flow rate: of $0.5 \mathrm{~mL} / \mathrm{min}$ Injection volume: $10 \mu \mathrm{L}$

Elution: gradient organic acids, anthocyanins, flavonoids, phenolic acid (cy-samb) flavonoids

[71]

$\mathrm{N} / \mathrm{A}$

protocatechuic acid 
Table 2. Cont.

\begin{tabular}{|c|c|c|c|c|}
\hline Pre-Analysis Step & $\begin{array}{l}\text { Determination } \\
\text { Method }\end{array}$ & Analysis Condition & Target Compound & Ref \\
\hline $\begin{array}{l}\text { Sonicated for } \\
\quad 30 \mathrm{~min}\end{array}$ & HPLC-MS & $\begin{array}{l}\text { Column: Polaris C18 Amide } \\
(250 \mathrm{~mm} \times 4.6 \mathrm{~mm}, 5 \mu \mathrm{m}) \\
\text { Mobile phase: (A) } 0.4 \% \text { formic } \\
\text { acid/water and (B) } 0.4 \% \text { formic } \\
\text { acid/acetonitrile } \\
\text { Elution: gradient }\end{array}$ & $\begin{array}{c}\text { Delphinidin (De) and } \\
\text { cyanidin (Cy), } \\
\text { delphinidin-3-sambubioside } \\
\text { (De-Sam) and } \\
\text { cyanidin-3sambubioside } \\
\text { (Cy-Sam) }\end{array}$ & [127] \\
\hline $\mathrm{N} / \mathrm{A}$ & $\begin{array}{l}\text { HPLC-DAD } \\
\text { HPLC-MS }\end{array}$ & $\begin{array}{c}\text { Column: Agilent ZORBAX Eclipse } \\
\text { Plus C18 } \\
(100 \mathrm{~mm} \times 4.5 \mathrm{~mm}, 3.5 \mu \mathrm{m}) \\
\text { Mobile phase: ethanol and tartaric } \\
\text { acid aqueous solution }(0.25 \mathrm{~mol} / \mathrm{L}) \\
\text { Elution: gradient } \\
\text { Column: Agilent ZORBAX Eclipse } \\
\text { Plus C18 (100 mm } \times 4.5 \mathrm{~mm}, 3.5 \mu \mathrm{m}) \\
\text { Mobile phase: ethanol and formic acid } \\
\text { aqueous solution }(0.1 \%, v / v) \\
\text { Elution: gradient }\end{array}$ & $\begin{array}{l}\text { Delphinidin-3-O-sambubioside } \\
\text { and cyanidin-3-O-sambubioside }\end{array}$ & [33] \\
\hline $\begin{array}{l}\text { Dissolved in } \\
\text { methanol }\end{array}$ & UPLC & $\begin{array}{c}\text { Column: UPLC BEH RP C18 } \\
(50 \mathrm{~mm} \times 2.1 \mathrm{~mm}, 1.7 \mu \mathrm{m}) \\
\text { Mobile phase: }(\mathrm{A}) 0.3 \% \text { phosphoric } \\
\text { acid in water (B) acetonitrile } \\
\text { Elution: gradient }\end{array}$ & $\begin{array}{l}\text { Anthocyanidins: delphinidin, } \\
\text { cyanidin, petunidin, peonidin, } \\
\text { pelargonidin, malvidin }\end{array}$ & [115] \\
\hline $\mathrm{N} / \mathrm{A}$ & $\begin{array}{l}\text { UPLC-DAD- } \\
\text { ESI/MS }\end{array}$ & $\begin{array}{c}\text { Column: Waters Spherisorb S3 ODS-2 } \\
\text { C18 }(150 \mathrm{~mm} \times 4.6 \mathrm{~mm}, 3 \mu \mathrm{m}) \\
\text { Mobile phase for anthocyanin } \\
\text { separation: }(\mathrm{A}) 0.1 \% \text { trifluoroacetic } \\
\text { acid in water (B) acetonitrile } \\
\text { Mobile phase for non-anthocyanin } \\
\text { separation: (A) } 0.1 \% \text { formic acid in } \\
\text { water (B) acetonitrile } \\
\text { Elution: gradient }\end{array}$ & $\begin{array}{l}\text { 3-O-caffeoylquinic acid, } \\
\text { 4-O-caffeoylquinic acid, } \\
\text { 5-O-caffeoylquinic acid, caffeic } \\
\text { acid, myricetin- } O \text {-sambubioside, } \\
\text { quercetin- } O \text {-sambubioside, } \\
\text { quercetin-3-O-rutinoside, } \\
\text { quercetin-3-O-glucoside, } \\
\text { kaempferol-3-O-rutinoside }\end{array}$ & [28] \\
\hline $\mathrm{N} / \mathrm{A}$ & $\begin{array}{l}\text { UPLC-DAD- } \\
\text { ESI/MS }\end{array}$ & $\begin{array}{c}\text { Column: Waters Spherisorb S3 ODS-2 } \\
\text { C18 }(150 \mathrm{~mm} \times 4.6 \mathrm{~mm}, 3 \mu \mathrm{m}) \\
\text { Mobile phase for anthocyanin } \\
\text { separation: }(\mathrm{A}) 0.1 \% \text { trifluoroacetic } \\
\text { acid in water (B) acetonitrile } \\
\text { Mobile phase for non-anthocyanin } \\
\text { separation: (A) } 0.1 \% \text { formic acid in } \\
\text { water (B) acetonitrile } \\
\text { Elution: gradient }\end{array}$ & $\begin{array}{l}\text { Anthocyanin and } \\
\text { non-anthocyanin }\end{array}$ & [99] \\
\hline $\mathrm{N} / \mathrm{A}$ & UPLC-MS/MS & $\begin{array}{c}\text { Column: RP HSS T3 C18 } \\
(100 \mathrm{~mm} \times 2.1 \mathrm{~mm}, 1.7 \mu \mathrm{m}) \\
\text { Mobile phase: }(\mathrm{A}) \text { formic acid } 0.1 \%(\mathrm{~B}) \\
\text { acetonitrile containing } 0.1 \% \\
\text { formic acid } \\
\text { Elution: gradient }\end{array}$ & $\begin{array}{l}36 \text { identified metabolites, } \\
\text { including flavonoids, } \\
\text { anthocyanins, phenolic and } \\
\text { organic acids }\end{array}$ & [38] \\
\hline
\end{tabular}


Table 2. Cont.

\begin{tabular}{|c|c|c|c|c|}
\hline Pre-Analysis Step & $\begin{array}{l}\text { Determination } \\
\text { Method }\end{array}$ & Analysis Condition & Target Compound & Ref \\
\hline $\mathrm{N} / \mathrm{A}$ & UPLC-MS/MS & $\begin{array}{c}\text { Column: BEH C18 } \\
(50 \mathrm{~mm} \times 2.1 \mathrm{~mm}, 1.7 \mu \mathrm{m}) \\
\text { Mobile phase: }(\mathrm{A}) \text { ultrapure water } \\
\text { acidified with } 0.1 \% \text { formic acid } \\
\text { (B) methanol } \\
\text { Elution: gradient }\end{array}$ & $\begin{array}{c}\text { Hydroxycitric acid, } \\
\text { caffeoylquinic acid, quercetin } \\
\text { 3-O-glycoside, cyanidin } \\
\text { 3-O-rutinoside, myricetin, rutin, } \\
\text { pelargodine, cyanidin, } \\
\text { delphinidin, 3-O-sambubioside } \\
\text { delphinidin and cyanidin } \\
\text { 3-O-sambubioside, quercetin } \\
\text { hexoside, quercetin, myricitin, } \\
\text { and 4-caffeoylquinic acid }\end{array}$ & [9] \\
\hline $\begin{array}{l}\text { The dried extract } \\
\text { was reconstituted in } \\
\text { the mobile phase } \\
\text { (water with } 0.1 \% \\
\text { formic acid) }\end{array}$ & $\begin{array}{l}\text { UPLC-QTOF-MS- } \\
\text { ESI }\end{array}$ & $\begin{array}{c}\text { Column: BEH C18 } \\
(100 \mathrm{~mm} \times 2.1 \mathrm{~mm}, 1.7 \mu \mathrm{m}) \\
\text { Mobile phase: }(\mathrm{A}) \text { water with } 0.1 \% \\
\text { formic acid (B) acetonitrile with } 0.1 \% \\
\text { formic acid } \\
\text { Elution: gradient }\end{array}$ & $\begin{array}{c}34 \text { identified extractable } \\
\text { polyphenols (EPP), hydrolysable } \\
\text { non-extractable polyphenols } \\
(\mathrm{NEPP}), \text { and } 2 \text { organic acids }\end{array}$ & [128] \\
\hline $\begin{array}{l}\text { The dried extract } \\
\text { was dissolved in } 1 \\
\text { mL methanol }\end{array}$ & UHPLC-MS/MS & $\begin{array}{c}\text { Column: N/A } \\
\text { Mobile phase: Formic acid } 0.1 \% \\
\text { Electrospray Ionization (ESI) (voltage } \\
3 \mathrm{kV} \text {; evaporation temperature } 250{ }^{\circ} \mathrm{C} \text {; } \\
\text { capillary temperature } \\
300^{\circ} \mathrm{C} \text {; nitrogen } 40 \text { psi, and Aux } 10 \\
\text { psi with argon gas) }\end{array}$ & $\begin{array}{c}\text { Myricetin, } \beta \text {-carotene, ascorbic } \\
\text { acid }\end{array}$ & [129] \\
\hline $\begin{array}{l}\text { The dried extract } \\
\text { was reconstituted in } \\
300 \mu \mathrm{L} \text { of methanol }\end{array}$ & $\begin{array}{l}\text { UHPLC-MS } \\
\text { NMR }\end{array}$ & $\begin{array}{l}\text { Column: Waters Acquity HSS T3 RP } \\
(150 \mathrm{~mm} \times 1 \mathrm{~mm}, 1.8 \mu \mathrm{m}) \\
\text { Mobile phase: }(\mathrm{A}) \text { water contain } 0.1 \% \\
(v / v) \text { formic acid }(\mathrm{B}) \text { acetonitrile } \\
\text { contain } 0.1 \%(v / v) \text { formic acid } \\
\text { Elution: gradient }\end{array}$ & $\begin{array}{c}33 \text { metabolites: sugars, } \\
\text { flavonoids, anthocyanins, and } \\
\text { phenolic and aliphatic organic } \\
\text { acids }\end{array}$ & [39] \\
\hline $\begin{array}{l}\text { Solid-phase } \\
\text { microextraction } \\
\quad(\mathrm{SPME})\end{array}$ & $\begin{array}{c}\text { GC } \\
\text { GC-MS }\end{array}$ & $\mathrm{N} / \mathrm{A}$ & Volatile compounds & [82] \\
\hline $\begin{array}{l}\text { The extract was } \\
\text { dried over } \\
\text { anhydrous sodium } \\
\text { sulfate and } \\
\text { concentrated to } \\
0.6 \mathrm{~mL} \text { on a } \\
\text { Kuderna-Danish } \\
\text { evaporator with a } \\
\text { 12-cm Vigreux } \\
\text { column and further } \\
\text { evaporated to } \\
\text { 0.2 mL with a } \\
\text { gentle nitrogen } \\
\text { stream }\end{array}$ & $\begin{array}{l}\text { GC-FID } \\
\text { GC-MS }\end{array}$ & $\begin{array}{c}\text { Column: AT- } 5 \mathrm{~ms} \\
(30 \mathrm{~m} \times 0.25 \mathrm{~mm}, 0.5 \mu \mathrm{m}) \text { or DB-Wax } \\
\text { column }(30 \mathrm{~m} \times 0.25 \mathrm{~mm}, 0.25 \mu \mathrm{m}) \\
\text { Split mode }(1: 50) \text { at } 250{ }^{\circ} \mathrm{C} \\
\text { Carrier gas: Helium } \\
\text { Flow rate: } 1 \mathrm{~mL} / \mathrm{min} \\
\text { Same condition with GC-FID }\end{array}$ & Volatile compounds & [105] \\
\hline $\begin{array}{l}\text { Solid-phase } \\
\text { microextraction } \\
\quad(\mathrm{SPME})\end{array}$ & GC-MS & $\begin{array}{c}\text { Column: Agilent J\&W DB-WAX } \\
\text { 122-7062 }(60 \mathrm{~m} \times 0.25 \mathrm{~mm}, 25 \mu \mathrm{m}) \\
\text { Split ratio of } 1: 10 \\
\text { Flow rate: } 1 \mathrm{~mL} / \mathrm{min} \\
\text { Carrier gas: Helium }\end{array}$ & Volatile compounds & [108] \\
\hline
\end{tabular}


Table 2. Cont.

\begin{tabular}{|c|c|c|c|c|}
\hline Pre-Analysis Step & $\begin{array}{c}\text { Determination } \\
\text { Method }\end{array}$ & Analysis Condition & Target Compound & Ref \\
\hline SPME & GC-MS & $\begin{array}{c}\text { Column: DB5-MS } \\
(30 \mathrm{~m} \times 0.25 \mathrm{~mm}, 0.25 \mu \mathrm{m}) \\
\text { Splitless mode for } 30 \mathrm{~s} \\
\text { Carrier gas: Helium } \\
\text { Flow rate: } 1 \mathrm{~mL} / \mathrm{min}\end{array}$ & Volatile compounds & [107] \\
\hline SPME & GC-MS & $\begin{array}{c}\text { Column: CP-Wax } 52 \mathrm{CB} \\
(30 \mathrm{~m} \times 0.25 \mathrm{~mm}, 0.25 \mu \mathrm{m}) \\
\text { Split mode }(1: 10) \\
\text { Initial temperature: } 60^{\circ} \mathrm{C}(2 \mathrm{~min}) \\
\text { Carrier gas: Nitrogen } \\
\text { Flow rate of } 1 \mathrm{~mL} / \mathrm{min}\end{array}$ & Volatile compounds & [106] \\
\hline SPME & GC-MS & $\begin{array}{c}\text { Column: HP5 }(30 \mathrm{~m} \times 0.25 \mathrm{~mm}, \\
0.25 \mu \mathrm{m} \text { and BPX70 }(70 \% \\
\text { Cyanopropyl-Polysylphenylene- } \\
\text { Siloxane) capillary column } \\
(50 \mathrm{~m} \times 0.22 \mathrm{~mm} \times 0.25 \mu \mathrm{m}) \\
\text { Carrier gas: Helium } \\
\text { Flow rate of } 1.7 \mathrm{~mL} / \mathrm{min} \\
\text { Initial temperature } 50^{\circ} \mathrm{C}\end{array}$ & Volatile compounds & [104] \\
\hline LLE & GC-MS-O & $\begin{array}{c}\text { Column: DB-Wax } \\
(30 \mathrm{~m} \times 0.25 \mathrm{~mm}, 0.5 \mu \mathrm{m}) \text { and a } \\
\text { Gerstel ODP-2 }(\text { Linthicum, MD) } \\
\text { sniffing port using deactivated } \\
\text { capillary column }(30 \mathrm{~cm}-0.25 \mathrm{~mm}) \\
\text { Flow rate of } 1.5 \mathrm{~mL} / \mathrm{min} \\
\text { Carrier gas: Helium }\end{array}$ & Volatile compounds & [130] \\
\hline $\begin{array}{l}\text { Dynamic headspace } \\
\text { sampling using } \\
\text { Tenax TA cold trap }\end{array}$ & $\begin{array}{c}\text { GC-MS } \\
\text { GC-O }\end{array}$ & $\begin{array}{c}\text { Column: DBWax } \\
(30 \mathrm{~m} \times 0.25 \mathrm{~mm}, 0.50 \mu \mathrm{m} \\
\text { Flow rate of } 1.4 \mathrm{~mL} \text { min } \\
\text { Carrier gas: Hydrogen } \\
\text { Column: DB-Wax column }(30 \mathrm{~m} \times \\
0.25 \mathrm{~mm} \text { i.d., } 0.5 \mu \mathrm{m} \text { film thickness }) \\
\text { Carrier gas: Helium } \\
\text { Flow rate: } 1 \mathrm{~mL} \text { min; split ratio, } 1: 20\end{array}$ & Volatile compounds & [131] \\
\hline
\end{tabular}

\begin{tabular}{|c|c|c|c|c|}
\hline $\mathrm{N} / \mathrm{A}$ & GC-MS & $\begin{array}{l}\text { Column: direct capillary column TG } \\
\text { ram negative } 5 \mathrm{MS}(30 \mathrm{~mm} \times 0.25 \mathrm{~mm} \text {, } \\
0.25 \mu \mathrm{m}) \\
\text { Carrier gas: Helium } \\
\text { Elution: gradient }\end{array}$ & $\begin{array}{l}\text { Hydrocarbons (alkan)-saturated } \\
\text { compounds, alcoholic } \\
\text { compounds, triazine derivatives, } \\
\text { unsat. alcoholic compounds, } \\
\text { unsat. ester, merceoto } \\
\text { compound, alkenes, primary } \\
\text { alcohols, natural product } \\
\text { (cholesterol) }\end{array}$ & [132] \\
\hline $\begin{array}{l}\text { The extract was } \\
\text { diluted in methanol }\end{array}$ & TLC & $\begin{array}{l}\text { mixture of chloroform }(\mathrm{CHCl} 3) \text { and } \\
\text { methanol }(\mathrm{CH} 3 \mathrm{OH}) \text { in ratio } 4: 1\end{array}$ & $\begin{array}{c}\text { glycosides, alkaloids, steroids, } \\
\text { triterpenoids, tannins, and } \\
\text { flavonoid }\end{array}$ & [111] \\
\hline $\mathrm{N} / \mathrm{A}$ & TLC & $\begin{array}{l}\text { Column: (silica gel G60 F254 TLC plates } \\
\text { of E. Merck, layer thickness } 0.2 \mathrm{~mm}) \\
\text { Mobile phased: butanol:acetic:water } \\
\text { (4:1:5) and methanol:water (95:5) } \\
\text { Wavelength: } 365-254 \mathrm{~nm}\end{array}$ & flavonoid & [112] \\
\hline
\end{tabular}




\section{Phenolic Compounds in Roselle}

\subsection{Total Phenolics}

Various studies of the phenolic compound content of roselle calyx tissue, identifying and quantifying the total and specific compounds, have been carried out over the last decade (Tables 1 and 2). Most of the studies were carried out by measuring the phenolic content as a response parameter of the total compounds, referred to as the total phenolic compounds (TPC), total flavonoid compounds (TFC), and total anthocyanin compounds (TAC). The list of studies reporting the phenolic compound contents as total compounds are shown in Table 3. Generally, the TPC was determined as gallic acid equivalents; TFC as quercetin, rutin, or catechin; and TAC as cyanidin-3-sambusioside (Cy-3-S) or delphinidin3-sambusioside (Dep-3-S).

From various reports of the total phenolic compound levels as TPC, TFC, and TAC, the differences between studies can be observed. Besides being influenced by sample conditions such as cultivars, growing environments, and processing treatments, the variations between studies might be caused by differences in analytical methods, extraction steps, and analytical conditions. The variety of samples, combined with processing conditions and analysis methods, caused difficulty in conducting direct comparisons between studies $[27,122,133,134]$. For example, in determining the TAC levels of a Sudanese cultivar with a dark-red calyx, one study reported a TAC level of $107.7 \mathrm{mg} / \mathrm{g}$ (expressed as cyanidin-3-sambubioside, Cy-3-S) [27], while, in their previous study, the TAC level of the same cultivar was reported as 32.96-mg Cy-3-S/g using a different analytical method [108]. Furthermore, a sample of a Sudan cultivar from Mexico was reported to have a value of 10.99-mg Cy-3-S/g [118], while a recent study from Mexico reported a TAC level of 6.56-mg Cy-3-S/g [98].

\subsubsection{Total Phenolic Compounds}

Comparative studies of the total phenolic compounds from roselle to other species have been reported [34,35,63,90,92,114,115,119,132,135-141]. Roselle was reported to have the highest level of TPC compared to cabbage (Brassica oleracea) and beet (Beta vulgaris) [132]. Other studies also reported that roselle had the highest TPC level compared to four five other edible flowers: carnations (Dianthus caryophyllus), sunflowers (Helianthus annuus), and purple and pink violets (Saintpaulia ionantha) [141]; ten traditional plants from the Andaman and Nicobar Islands [140]; and ten common edible plant species found in the Mediterranean region [35]. As presented in Table 3, the reported TPC value of roselle calyx ranged from 0.78 to $291.78 \mathrm{mg} \mathrm{GAE} / \mathrm{g}$, in which the lowest value was found in a white-calyx variety from Sudan [108], while the highest value found in "Cruza Negra", a cultivar from Mexico [122].

Generally, the TPC value from a dark-red calyx roselle cultivar-namely, a "white Al-Rahad" variety from Sudan and "Cruza Negra" from Mexico-had a higher value compared to roselle cultivars with a light-red calyx "Criolla" or white calyx "Blanca" from Mexico. Therefore, it is assumed that the presence of pigment compounds in roselle cultivars-specifically, the anthocyanin content-might influence the TPC value. However, the cultivar with highest TAC value did not necessarily have the highest TPC value $[68,142]$. It was strongly suspected that white or bright-colored calyx cultivars with no detectable TAC or only trace levels might have other phenolic compounds at high levels-for example, TFC; therefore, even a white calyx cultivar could have a high TPC value $[29,108]$.

A study investigating the effects of the genotype, environmental conditions, and their interactions on the phenolic contents of 53 roselle cultivars from several regions in Mexico revealed that the genotype alone accounted for $44 \%$ of the variation in TPC levels, followed by interactions between the genotypes and environmental conditions [142]. Regarding the environmental conditions-namely, agricultural practices-a study reported that the TPC values of roselle plants might be increased by improving the nutrient balance and physical properties of the soil. However, no significant difference was detected between the treatments and control [145]. In addition to environmental conditions, an increase in 
the $\mathrm{CO}_{2}$ pressure strongly correlated with higher TPC levels in the calyx. Differences in the $\mathrm{CO}_{2}$ pressure in the plant environment might cause differences in the plant growth and production of carbon-based compounds, including phenolic compounds [31].

In terms of postharvest processing conditions, the drying and storage conditions of calyx roselle will also affect the TPC levels. Several studies investigated the retention of TPC in the calyx after extraction or calyx byproducts after extraction with a combination of various drying techniques [117,146-148]. One of the studies reported that the TPC value was higher with a lower drying temperature and lower airflow velocity, indicating that the phenolic compounds were sensitive to elevated temperatures [117]. The stability of the TPC in roselle towards various exposures in storage-namely, temperature, light, and $\mathrm{pH}$-were also investigated in other studies [149,150]. During 22 storage days at a temperature of 4 ${ }^{\circ} \mathrm{C}$ without light exposure, the TPC content of the extracts in ethanol was degraded by $15 \%$, and when stored at a temperature of $25{ }^{\circ} \mathrm{C}$, the degradation increased to $23 \%$ and was even higher with light exposure [149]. Another study investigated the influence of the water activity or $a_{\mathrm{w}}$ on the phenolic compound stability during storage [150]. They reported that the TPC content of roselle calyx was stable at a low $a_{\mathrm{w}}$; thus, they found a value of 0.288 as the most stable value of $a_{\mathrm{w}}$ where the prevention of the polyphenol compound breakdown could best be achieved. However, $a_{\mathrm{w}}$ values above 0.329 increased the phenolic content extractability, but the degradation of the phenolic components also increased due to the release of more free phenolic compounds and enzymatic activity by swelling the surrounding tissues and dissolution of the components in the matrix.

Table 3. Total concentration of phenolic compounds from roselle. Abbreviations: N/A: not available, TPC: total phenolic compound, TFC: total flavonoid compound, TAC: total anthocyanin compound, L: lowest value detected, and H: highest value detected.

\begin{tabular}{|c|c|c|c|c|c|}
\hline Country & TPC & TFC & TAC & Sample & Ref \\
\hline Sudan & $\mathrm{N} / \mathrm{A}$ & $\mathrm{N} / \mathrm{A}$ & $\begin{array}{c}\text { L: } 0.02 \text { mg Cy-3-G/g } \\
\text { (Al-Ubayyid) } \\
\text { H: } 107.7 \text { mg Cy-3-G/g } \\
\text { (Al-Rahad) }\end{array}$ & $\begin{array}{c}8 \text { cultivars } \\
\text { Al-Ubayyid (white), Al-Gezira, Nyala, } \\
\text { Al-Rahad, Al-Ubayyid, Kaduqli, El } \\
\text { Geneina, Al-Fashir, China }\end{array}$ & [27] \\
\hline Senegal & $\begin{array}{c}\text { L: } 19.30 \mathrm{mg} \\
\text { GAE/g (Thai) } \\
\text { H: } 28.20 \mathrm{mg} \\
\text { GAE/g } \\
\text { (Vimto) }\end{array}$ & $\mathrm{N} / \mathrm{A}$ & $\begin{array}{c}\text { L: } 8.20 \text { mg D-3-D-X/g (Koor) } \\
\text { H: } 17.30 \text { mg D-3-D-X/g } \\
\text { (CLT92) }\end{array}$ & $\begin{array}{c}4 \text { cultivars } \\
\text { (Vimto, Koor, Thaï, CLT92) }\end{array}$ & [133] \\
\hline Thailand & $\mathrm{N} / \mathrm{A}$ & $\mathrm{N} / \mathrm{A}$ & $\begin{array}{c}\text { L: } 0.02 \text { mg Cy-3-G/g (White) } \\
\text { H: } 19.48 \text { mg Cy-3-G/g } \\
\text { (HAC, Dark-purple closed } \\
\text { calyx) }\end{array}$ & $\begin{array}{c}15 \text { genotypes } \\
\text { Purple-Jumbo; Red-Jumbo; } \\
\text { Pink-Jumbo; White calyx; } \\
\text { Purple-Jumbo-Opened; } \\
\text { Red-Jumbo-Opened; } \\
\text { Pink-Jumbo-Opened; } \\
\text { Purple-Jumbo-Closed; } \\
\text { Red-Jumbo-Closed; } \\
\text { Pink-Jumbo-Closed; } \\
\text { Dark-purple-Closed; } \\
\text { Dark-purple-Opened; Purple } \\
\text { calyx-5-lobed leaf; Dark-red; } \\
\text { Orange-Red }\end{array}$ & [134] \\
\hline
\end{tabular}


Table 3. Cont.

\begin{tabular}{|c|c|c|c|c|c|}
\hline Country & TPC & TFC & TAC & Sample & Ref \\
\hline Mexico & $\begin{array}{c}\text { L: } 0.14 \mathrm{mg} \\
\text { GAE/g } \\
\text { (UAN16-2) } \\
\text { H: } 291.78 \mathrm{mg} \\
\text { GAE/g } \\
\text { (Cruza } \\
\text { Negra) }\end{array}$ & $\mathrm{N} / \mathrm{A}$ & $\begin{array}{c}\text { L: } 10.00 \text { Cy-3-G/g } \\
\text { (UAN25-1) } \\
\text { H: } 180.00 \text { Cy-3-G/g (Cruza } \\
\text { Negra) }\end{array}$ & $\begin{array}{c}6 \text { cultivars } \\
\text { Cruza Negra, Criolla Huajicori, } \\
\text { UAN25-1, UAN16-2, 4Q4, } \\
\text { UAN6-Puga }\end{array}$ & [29] \\
\hline Nigeria & $\begin{array}{c}\text { L: } 36.04 \mathrm{mg} \\
\text { GAE/g } \\
\text { (dark-red wet } \\
\text { season) } \\
\text { H: } 38.00 \mathrm{mg} \\
\text { GAE/g } \\
\text { (dark red-dry } \\
\text { season) }\end{array}$ & $\mathrm{N} / \mathrm{A}$ & $\begin{array}{l}\text { L: } 19.57 \mathrm{mg} / \mathrm{g} \text { (dark-red wet } \\
\text { season) } \\
\mathrm{H:} 27.10 \mathrm{mg} / \mathrm{g} \text { (dark-red dry } \\
\text { season) }\end{array}$ & $\begin{array}{l}\text { dark red cultivar in wet and dry } \\
\text { season }\end{array}$ & [30] \\
\hline Mexico & $\begin{array}{c}\text { L: } 11.64 \mathrm{mg} \\
\text { GAE/g } \\
\text { (Alma } \\
\text { Blanca) } \\
\text { H: } 17.57 \mathrm{mg} \\
\text { GAE/g } \\
\text { (Criolla) }\end{array}$ & $\begin{array}{c}\mathrm{L}: 0.58 \mathrm{mg} \\
\mathrm{QE} / \mathrm{g}, 2.86 \\
\mathrm{mg} \mathrm{CE} / \mathrm{g} \\
\text { (Organic } \\
\text { Criolla) } \\
\mathrm{H}: 1.43 \mathrm{mg} \\
\mathrm{QE} / \mathrm{g}, 7.84 \\
\mathrm{mg} \mathrm{CE} / \mathrm{g} \\
\text { (Criolla) }\end{array}$ & $\begin{array}{c}\text { L: } 0.12 \text { mg Cy-3-G/g (Alma } \\
\text { Blanca) } \\
\text { H: } 3.98 \text { mg Cy-3-G/g } \\
\text { (Criolla) }\end{array}$ & $\begin{array}{c}2 \text { cultivars } \\
\text { Criolla, Alma Blanca, Criolla variety, } \\
\text { Organic Criolla }\end{array}$ & [68] \\
\hline Sudan & $\begin{array}{c}\text { L: } 0.80 \mathrm{mg} \\
\text { GAE/g } \\
\text { (White) } \\
\text { H: } 1.48 \mathrm{mg} \\
\text { GAE/g } \\
\text { (Al-Rahad) }\end{array}$ & $\begin{array}{c}\text { L: } 0.18 \mathrm{mg} \\
\text { QE/g (White) } \\
\text { H: } 0.27 \mathrm{mg} \\
\text { QE/g } \\
\text { (Al-Rahad) }\end{array}$ & $\begin{array}{c}\text { L: } 0.00 \text { mg Cy-3-G/g (White) } \\
\text { H: } 32.96 \text { mg Cy-3-G/g } \\
\text { (Al-Rahad) }\end{array}$ & $\begin{array}{l}4 \text { cultivars } \\
\text { White, Al-Gezira, Al-Fashir, } \\
\text { Al-Rahad }\end{array}$ & [108] \\
\hline Mexico & $\begin{array}{c}\text { L: } 6.20 \mathrm{mg} \\
\text { GAE/g } \\
\text { (DMS) } \\
\text { H: } 36.40 \mathrm{mg} \\
\text { GAE/g } \\
\text { (UAN21) }\end{array}$ & $\mathrm{N} / \mathrm{A}$ & $\begin{array}{c}\text { L: } 1.48 \text { mg Cy-3-G/g (DMS) } \\
\text { H: 7.57 mg Cy-3-G/g } \\
\text { (UAN18) }\end{array}$ & $\begin{array}{c}53 \text { genotypes } \\
\text { Tempranilla Negra, Tempranilla Flor, } \\
\text { Colima, Jersey Acriollada, Criolla } \\
\text { Roja Violeta, Criolla Huajicor, Negra } \\
\text { UAN, Criolla Morada, UAN5, Criolla } \\
\text { Súper Precoz, Criolla Puebla Precoz, } \\
\text { Criolla Precoz, Negra Quiviquinta, } \\
\text { China, UAN 6 Puga, UAN 31, UAN } \\
\text { 6-1, UAN 16-2, UAN 6 Novillero, } \\
\text { MoradaXRoja, UAN 25, UAN 7, } \\
\text { Tempranilla Roja, UAN 23, UAN 11, } \\
\text { UAN 24, UAN 21, UAN 8, UAN 13, } \\
\text { UAN 17, UAN 26, UAN 27, UAN } \\
\text { 12-1, UAN 15, UAN 24-1, UAN 12, } \\
\text { UAN 20, UAN 22, UAN 10-1, UAN } \\
\text { 29, UAN 19, UAN 30, UAN 16, UAN } \\
\text { 18, UAN 21-1, UAN 10-2, 2MQ2, 3Q3, } \\
\text { 6Q6, 7Q7, 10, CONEJA, Q12, DMS }\end{array}$ & [142] \\
\hline USA & $\begin{array}{c}\text { L: } 15.92 \mathrm{mg} \\
\text { GAE/g } \\
\text { (Jamaica) } \\
\text { H: } 19.25 \mathrm{mg} \\
\text { GAE/g } \\
\text { (Senegal) }\end{array}$ & $\mathrm{N} / \mathrm{A}$ & $\mathrm{N} / \mathrm{A}$ & $\begin{array}{c}3 \text { accessions from Jamaica, Senegal, } \\
\text { Malaysia }\end{array}$ & [127] \\
\hline
\end{tabular}


Table 3. Cont.

\begin{tabular}{|c|c|c|c|c|c|}
\hline Country & TPC & TFC & TAC & Sample & Ref \\
\hline Mexico & $\mathrm{N} / \mathrm{A}$ & $\mathrm{N} / \mathrm{A}$ & $\begin{array}{c}\text { L: } 1.71 \mathrm{mg} \text { Cy-3-S/g, mg } \\
\text { Dep-sam/g (Rosa) } \\
\text { H: } 6.56 \text { mg Cy-3-S/g, 23.74 } \\
\text { mg Dep-sam/g (Sudan) } \\
\text { nd: Blanca }\end{array}$ & $\begin{array}{c}4 \text { cultivars } \\
\text { Negra, Sudan, Rosa, Blanca }\end{array}$ & [106] \\
\hline Mexico & $\begin{array}{c}\text { L: } 24.00 \mathrm{mg} \\
\text { GAE/g } \\
\text { (Blanca) } \\
\text { H: } 100.00 \mathrm{mg} \\
\text { GAE/g } \\
\text { (Real) }\end{array}$ & $\begin{array}{c}\text { L: } 4.19 \mathrm{mg} \\
\text { QE/g (Talpa) } \\
\text { H: } 22.6 \mathrm{mg} \\
\text { QE/g } \\
\text { (Reyna) }\end{array}$ & $\begin{array}{c}\text { L: } 0.00 \text { mg Cy-cl/g, } 0.00 \\
\mathrm{mgDep}-\mathrm{cl} / \mathrm{g} \text { (JB 00001 SM) } \\
\mathrm{H}: 8.73 \mathrm{mg} \mathrm{Cy-cl/g,} 35.35 \\
\mathrm{mgDep}-\mathrm{cl} / \mathrm{g} \text { (Tempranilla) } \\
\text { nd: Reyna }\end{array}$ & $\begin{array}{l}25 \text { cultivars } \\
\text { Americana, Tepalcatepec, Diamante, } \\
\text { Colima, Tempranilla, Talpa, Violenta, } \\
\text { Quesería, CriollaTala, Tecoman, El } \\
\text { Bordo, Tempranilla, Sudan, Media } \\
\text { Luna, Pisila/Colima, JB 00001 SM, } \\
\text { JCP 0001 T, Mutante Blanca, JJ 00001 } \\
\text { SM, JR 00001 C, Americana, Puerta de } \\
\text { Anzar, Variedad Blanca, Real, Reyna }\end{array}$ & [122] \\
\hline India & $\begin{array}{l}\text { L: } 134.32 \mathrm{mg} \\
\text { GAE/g (Red) } \\
\text { H: } 154.60 \mathrm{mg} \\
\text { GAE/g } \\
\text { (White) }\end{array}$ & $\mathrm{N} / \mathrm{A}$ & $\mathrm{N} / \mathrm{A}$ & $\begin{array}{l}2 \text { cultivars } \\
\text { red and white }\end{array}$ & [143] \\
\hline Mexico & $\begin{array}{c}\text { L: } 13.50 \mathrm{mg} \\
\text { GAE/g } \\
\text { (Alma Blanca, } \\
\text { White) } \\
\text { H: } 36.50 \mathrm{mg} \\
\text { GAE/g } \\
\text { (Sudan, } \\
\text { Dark-red) }\end{array}$ & $\mathrm{N} / \mathrm{A}$ & $\begin{array}{c}\text { L: } 0.20 \text { mg Cy-3-G/g Alma } \\
\text { (Blanca, white) } \\
\text { H: } 10.99 \text { mg Cy-3-G/g } \\
\text { (Sudan, dark-red) }\end{array}$ & $\begin{array}{c}3 \text { cultivars } \\
\text { Alma Blanca (white), Criolla Nayarit } \\
\text { (light red), Sudan (dark red) }\end{array}$ & [118] \\
\hline Mexico & $\begin{array}{l}\text { L: } 2.80 \mathrm{mg} \\
\text { GAE/g } \\
\text { (Criolla) } \\
\mathrm{H}: 6.80 \mathrm{mg} \\
\mathrm{GAE} / \mathrm{g} \\
\text { (China) }\end{array}$ & $\mathrm{N} / \mathrm{A}$ & $\mathrm{N} / \mathrm{A}$ & $\begin{array}{l}4 \text { cultivars } \\
\text { Criolla, Rosalis, Tecoanapa, China }\end{array}$ & [144] \\
\hline Mexico & $\mathrm{N} / \mathrm{A}$ & $\mathrm{N} / \mathrm{A}$ & $\begin{array}{c}\text { L: } 1.14 \text { mg Cy-3-G/g (China) } \\
\text { vH: } 7.24 \text { mg Cy-3-G/g } \\
\text { (Reyna) }\end{array}$ & $\begin{array}{l}3 \text { cultivars in different maturing } \\
\text { stages } \\
\text { Criolla/Creole, Reina/Queen, China }\end{array}$ & [93] \\
\hline Turkey & $\begin{array}{l}\text { L: } 16.40 \mathrm{mg} \\
\text { GAE/g } \\
\text { (Turkey/Kızılay) } \\
\text { H: } 49.1 \mathrm{mg} \\
\text { GAE/g } \\
\text { (Turkey/ } \\
\text { Söğ̈ütözü) }\end{array}$ & $\mathrm{N} / \mathrm{A}$ & $\mathrm{N} / \mathrm{A}$ & $\begin{array}{c}8 \text { regions } \\
\text { Kızılay, Söğütözü, Tunalı Hilmi, } \\
\text { Kızılay, Ulus, Kızılay, Ulus, Söğütözü }\end{array}$ & [36] \\
\hline
\end{tabular}

\subsubsection{Total Flavonoid Compounds}

Roselle was reported to have the highest levels of TFC compared to cabbage (Brassica oleracea) and beets (Beta vulgaris) [132]. Several studies conducting comparisons of the TFC values of roselle calyx among cultivars $[10,108,122]$ revealed a range of TFC values from 0.1805-22.6 mg QE/g (Table 3), with the lowest value in "White" from Sudan [27] and the highest value in "Reyna", a cultivar from Mexico [122]. Unlike the TPC value, a roselle cultivar with a darker calyx color did not necessarily correspond to a high TFC value. This can be seen with the cultivar "Alma Blanca" with a white calyx color (without red pigment) that was reported to have a higher value of TFC (143.23 mg QE/g) compared to "Organic Criolla" with a light-red calyx color $(57.57 \mathrm{mg}$ QE/g) [68]. Similar results 
were also reported by another study; "Criolla Tala" had a lower TFC value (5.3 mg QE/g) compared to "Mutante Blanca" (12.72 mg QE/g) [122].

The effects of environmental and processing conditions on TFC levels exhibited similar tendencies to the TPC - namely, soil conditions and drying, respectively. Improving the soil's physical properties and nutrient balance was presumed to increase the flavonoid content, yet no significant difference was detected between the control and roselle plants treated with fertilizers [145]. One study investigating the effect of drying on calyx byproducts on the TFC levels reported that the TFC retention levels had the same inclination as the TPC retention levels [147]. Similarly, regarding the effects of the roselle calyx tissue particle size on the extracted components, it was revealed that the particle size resulting in a higher TPC value was also reported to have a higher TFC value [55,72].

\subsubsection{Total Anthocyanin Compounds}

The anthocyanin content associated with the attractive color of roselle calyx, together with the other phenolic compounds, made up a crucial quality of the calyx [29]. Roselle was reported to have the highest levels of TAC compared to cabbage (Brassica oleracea) and beets (Beta vulgaris) [132]. Roselle also had the highest levels compared to two other anthocyanin source plants: Malabar melastome (Melastoma malabathricum) and sweet potatoes (Ipomoea batatas) [138], and as infusion drinks compared to green and black teas (Camellia sinensis), roselle tea had the highest level of TAC [34]. Comparison studies of roselle cultivars from various regions around the globe (Sudan [27], Mexico [122], Senegal [133], Thailand [134], Mexico [93,106,118,122,142], and India [151]) compiled an overall TAC level ranging from 0.00 to $180.0 \mathrm{mg}$ Cy-3-G/g. The lowest value was found in a white calyx cultivar in which anthocyanin compounds were barely detected. The highest value of TAC reported was in "Cruza Negra" from Mexico [122]. Similar to the TPC value, the darker the calyx color, the higher the TAC value compared to a calyx with a lighter color or white color, due to the absence of anthocyanins [27,122].

Roselle genotypes reportedly accounted for $60-80 \%$ of the variations in calyx colors and $76 \%$ of the variations in the TAC values [142]. The interaction between the genotype and environment accounted for $62 \%$ of the variation in the calyx color tone, which was influenced by the presence of anthocyanins [142]. Similar to the TPC value, the TAC values were also influenced by the growing environment of the plants-namely, an increase in the ambient $\mathrm{CO}_{2}$ content resulted in calyces with higher TAC levels [31]. Another study reported that spraying copper on roselle leaves at a certain dose and frequency significantly increased the nutrient contents and levels of the TAC in the calyx without affecting the yield of the dry calyx [152]. Differences in the calyx maturity at harvest were also found to influence the variations in the TAC values between cultivars [93].

The influence of the processing conditions on the calyx, such as the administration of hot [117] and cold temperatures [153], also reportedly created variations in the TAC values. In contrast to a lower temperature and lower drying air velocity, a higher temperature (up to $80^{\circ} \mathrm{C}$ ) during drying resulted in a higher polymeric color, which suggested that the increase in drying temperature might facilitate the polymerization of anthocyanins and speed up the loss of the moisture content. The polymeric color indicates the formation of anthocyanin complexes with other phenolics that might provide greater stability [117]. In a study of roselle juice production from calyces with variations in a cold temperature treatment $\left(5^{\circ} \mathrm{C},-19{ }^{\circ} \mathrm{C}\right.$, and $\left.-80^{\circ} \mathrm{C}\right)$, the roselle juice with the lowest TAC value was obtained from calyx tissue stored at a freezing temperature $\left(-80^{\circ} \mathrm{C}\right)$ due to the changes in the $\mathrm{pH}$ caused by salt precipitates in condensed solutions. Preserving other nutritional attributes such as ascorbic acid, the cold temperature treatment also affected the sensory value and the resultant color of the juice [153]. Furthermore, a significant correlation was reported between the sensory perception and TAC value of roselle beverages [154].

Various studies have also been conducted to examine the stability of anthocyanins toward various conditions - namely, the $\mathrm{pH}$ [138], temperature [61], light exposure, or a combination of these conditions $[76,94,149,155]$. Generally, anthocyanins in aqueous or 
ethanolic extracts tend to be stable at low temperatures, low light exposure, and low $\mathrm{pH}$. Furthermore, it was also reported that anthocyanins from roselle at low $\mathrm{pH}$ showed high stability against temperature changes up to $60^{\circ} \mathrm{C}$ and increased anthocyanin degradation rates between $80{ }^{\circ} \mathrm{C}$ and $100{ }^{\circ} \mathrm{C}$ [76]. The stability of anthocyanins against digestive enzymes was also carried out through in vitro studies. As a result, anthocyanin degradation in the extract was reported as $34.3 \%$ and $64.49 \%$ after in vitro simulations of digestive and colonic fermentation, respectively. The high degradation of anthocyanins was related to its sensitivity to alkaline conditions in the gastrointestinal tract, the secretion of bile salts, and various digestive enzymes [9].

\subsection{Phenolic Acids}

Roselle phenolic acids in the forms of hydroxybenzoic acid (gallic acid, protocatechuic acid, syringic acid, vanillic acid, genistic acid, and 4-hydroxybenzoic acid) and hydroxycinnamic acid (chlorogenic acid, caffeic acid, ferulic acid, $p$-coumaric acid, 3- $p$-coumaroylquinic acid, 5- $p$-coumaroylquinic acid, 3-O-caffeoylquinic acid, 4-O-caffeoylquinic acid, and 5-Ocaffeoylquinic acid) have been quantified in various studies, as presented in Table 4. Other phenolic acids identified in roselle calyx were neochlorogenic acid, cryptochlorogenic acid, methyl digallate, methyl chlorogenate, dihydroferulic acid-4-O-glucuronide, methyl chlorogenate isomer II, 5-O-caffeoyl shikimic acid, ethyl chlorogenate, and ethyl chlorogenate isomer II [12].

Protocatechuic acid and gallic acid were also reported as the major compounds found in roselle, and their levels in the flower calyx had the highest values compared to the nine other plants from the Mediterranean region [35]. The level of protocatechuic acid in roselle was also reported to have the highest value $(163.2 \mathrm{mg} / 100 \mathrm{~g}$ for roselle) compared to the other edible flowers-namely, rose (Rosa hybrida) and camellia flowers (Camellia japonica) [90]. The protocatechuic and caffeic acid contents were reported higher in white calyx roselle than in the red calyx roselle [118]. In acidified-methanolic roselle calyx extract, 3-caffeoylquinic acid was reported as a major phenolic compound [102], while in the acidified-aqueous extract, the major compounds were chlorogenic, $p$-coumaric, and caffeic acids [92].

Some studies reported the effect of environmental conditions and postharvest processing on the level of individual phenolic acid contents [30,31,46]. The levels of chlorogenic and caffeic acids in roselle calyx were higher in the growing environments with higher ambient $\mathrm{CO}_{2}$ contents than the normal conditions [31]. The contents of caffeic acid, gallic acid, and 3-O-caffeoylquinic acid in roselle were reported to be higher in the rainy season, while the content of protocatechuic acid was lower in the dry season [30]. An effect of postharvest drying treatments on the levels of protocatechuic acid was reported to have high levels in a drying treatment with a solar greenhouse and intermittent heat pumps compared to hot air and heat pumps [46].

The extraction conditions also affect the content of phenolic acids. Using ethanol as the extraction solvent resulted in an extract with higher levels of gallic acid, chlorogenic acid, quercetin, caffeic acid, and ferulic acid compared to ethyl acetate [81]. Meanwhile, from the results of a comparison study of the conventional maceration and ultrasoundassisted extraction (UAE) methods, the extract produced from the UAE method contained 4-caffeoylquinic acid, which was higher than conventional maceration [9]. 


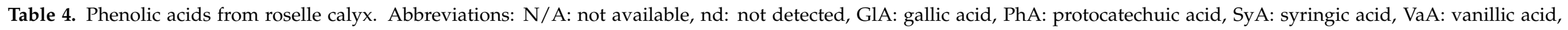

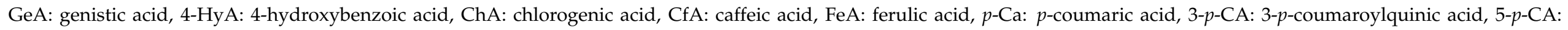
5-p-coumaroylquinic acid, 3-O-CfA: 3-O-caffeoylquinic acid, 4-O-CfA: 4-O-caffeoylquinic acid, and 5-CfA: 5-caffeoylquinic acid.

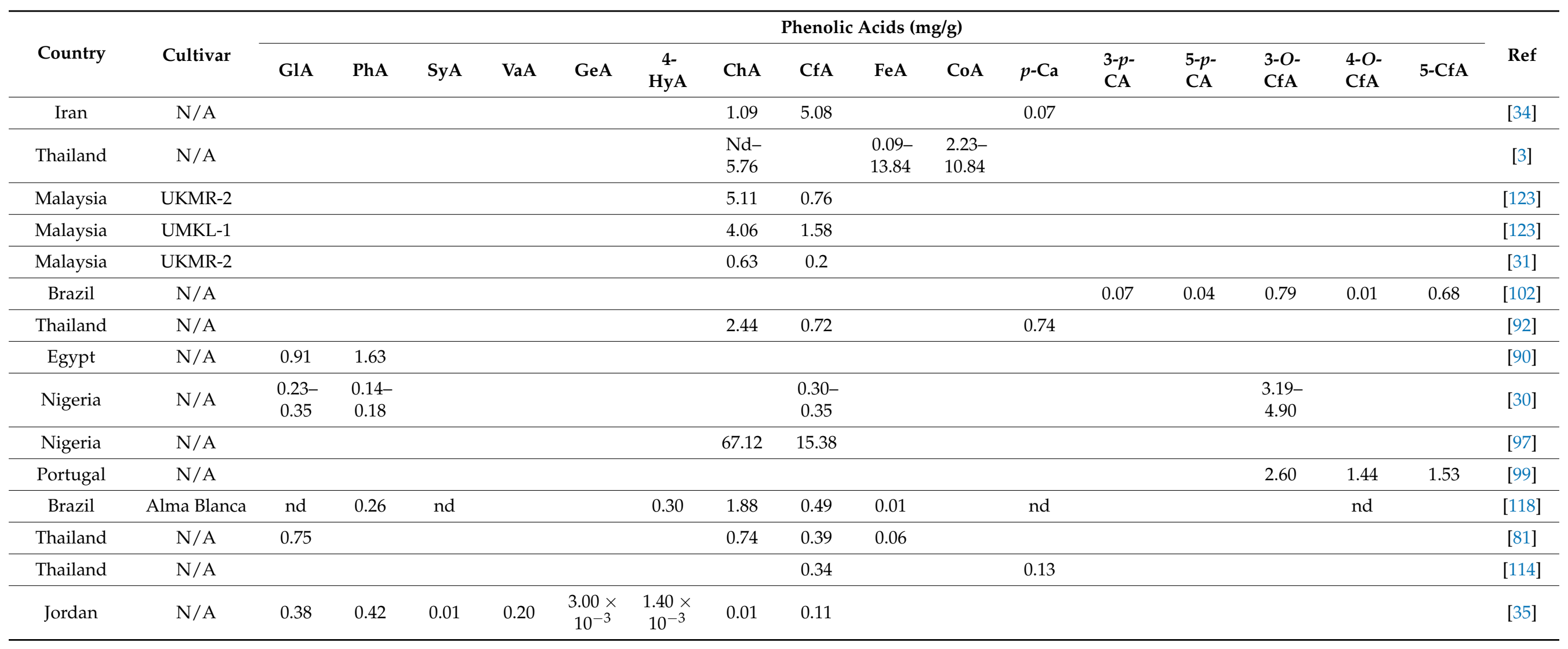


The isolation of individual phenolic acids from roselle extract and their pharmacological activities have been reported in several studies-namely, protocatechuic acids for their anti-urease activity [126] and gallic acid-protocatechuic acids for their potential antidiabetic effects [113]. Other phenolic acids reported to have antidiabetic potential were caffeic acid, p-coumaric acid [114], and rosmarinic acid [57]. Ferulic acid and chlorogenate esters found in methanolic roselle extract are reported to have antihypertensive potential through angiotensin-converting enzyme inhibitory (ACEI) activity [38]. Chlorogenic acids in a roselle aqueous extract were reported to have anti-hyperlipidemia potential through in vivo studies using animal trials [116]. Other studies also reported the results of studies of extracts containing chlorogenic $(67.12 \mathrm{mg} / \mathrm{g})$ and caffeic acids $(15.38 \mathrm{mg} / \mathrm{g})$ having neuroprotective activity through testing the activity of acetylcholinesterase (AChE), butyrylcholinesterase (BChE), monoamine oxidase (MAO), and ecto-5-nucleotidase (E-NTDase) [97].

\subsection{Flavonoids}

The flavonoids found in roselle were in the form of flavan-3-ols (epicatechin, (+)catechin, (-)-epigallocatechin, and (-)-epigallocatechin gallate); flavonols (kaempferol and kaempferol 3-O-rutinoside); myricetin (myricetin 3-sambubioside); quercetins (quercetin 3O-glucoside, quercetin 3-sambubioside, quercetin 3-rutinoside, and quercetin pentosylhexoside); rutin; quercitrin; and flavanones (hesperitin and hesperidin), as presented in Table 5. The most frequent reported flavonoids in roselle calyx tissue were quercetins [3,34,81,99,102]. Gossypetin, quercetin, sabdaretin, and hibiscetin are major compounds identified among the flavonoids found in an acidified-methanol extract [121].

Differences in the molecular regulatory process were assumed to be the cause of the higher level of flavanols detected in white calyx roselle "Blanca" compared to several red calyx cultivars [98]. Other factors that might cause variations in individual flavonoid contents in roselle calyx were the growing season and drying processes [30,46]. Myricetin 3$\mathrm{O}$-arabinogalactoside and quercetin-3-O-sambubioside were reported to have higher values when planted in the wet season than in the dry season [30]. At postharvest processing, the catechin content of roselle calyx after a drying process using a heat pump was higher than hot air drying, solar greenhouse drying, and solar greenhouse with intermittent heat pump drying [46].

The functional properties of flavonoids isolated from roselle calyx have also been investigated -namely, the antianxiety activity and antidepressant activity of gossypetin [40]. Kaempferol in a methanolic extract was confirmed as a potential antihypertensive through ACEI activity [38]. Quercetin functions as a diuretic through a oxide nitric mechanism that will increase the renal vasorelaxation [156] and as an antidiabetic through the inhibition of $\alpha$-amylase and $\alpha$-glucosidase activity [57]. Myricetin has potential as an antiaging agent [129]. In addition, extracts containing catechin, quercetin, and rutin were reported to have neuroprotective potential [97].

\section{Anthocyanins}

The isolation of individual anthocyanin compounds from roselle calyx has been conducted in several studies [71,86-88], revealing glycosylated anthocyanins with three or more sugar moieties [71] and octanoyl derivatives delphinidin-3-O-sambubioside and cyanidin-3-O-sambubioside [88], as presented in Table 6. Cyanidin-3-O-sambubioside and delphinidin-3-O-sambubioside were reported as two major anthocyanin compounds of roselle calyx $[33,58,71,83,86,115,121,123]$. 


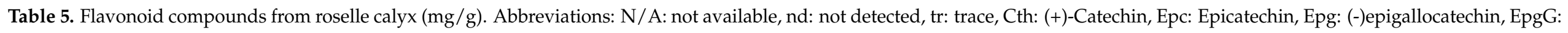

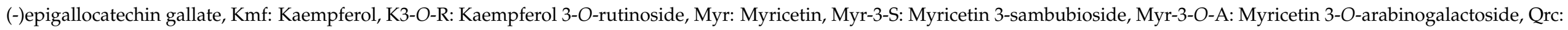

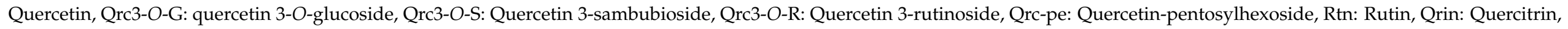
Hptn: Hesperitin, and Hpdn: Hesperidin.

\begin{tabular}{|c|c|c|c|c|c|c|c|c|c|c|c|c|c|c|c|c|c|c|c|}
\hline \multirow[b]{2}{*}{ Country } & \multicolumn{18}{|c|}{ Flavonoids } & \multirow[b]{2}{*}{ Ref } \\
\hline & Cth & Epc & Epg & EpgG & $\mathrm{Kmf}$ & $\begin{array}{l}\text { K3- } \\
\text { O-R }\end{array}$ & Myr & Myr-3-S & $\begin{array}{l}\text { Myr-3- } \\
\text { O-AS }\end{array}$ & Qrc & $\begin{array}{l}\text { Qrc3- } \\
O-G\end{array}$ & Qrc3-O-S & $\begin{array}{l}\text { Qrc3- } \\
O-\mathrm{R}\end{array}$ & $\begin{array}{l}\text { Qrc- } \\
\text { pe }\end{array}$ & Rtn & Qrin & Hptn & Hpdn & \\
\hline Iran & 0.92 & & & & & & & & & 0.16 & & & & & & & 0.51 & 0.14 & [34] \\
\hline Thailand & & & & & & & & & & $0.57-2.03$ & & & & & & & & & [3] \\
\hline Brazil & & & & & & 0.02 & & & & 0.04 & & 0.01 & 0.09 & 0.08 & & & & & [102] \\
\hline Nigeria & & & & & & & & $0.28-0.35$ & & & & $0.21-0.24$ & & & & & & & [30] \\
\hline Portugal & & 1.03 & & & & $\operatorname{tr}$ & & & & 0.96 & & $\operatorname{tr}$ & & 1.07 & & & & & [99] \\
\hline Thailand & & & & & & & & & & 0.45 & & & & & & & & & [81] \\
\hline
\end{tabular}




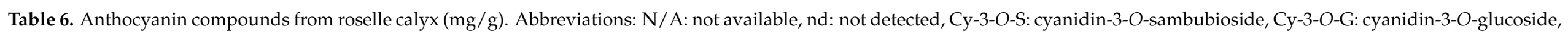

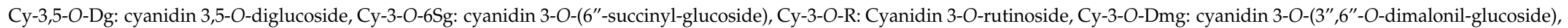

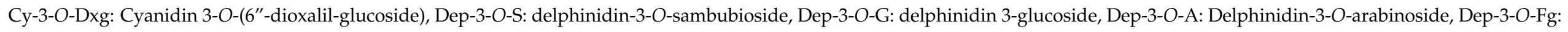

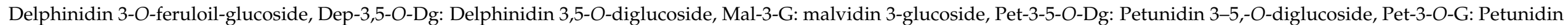

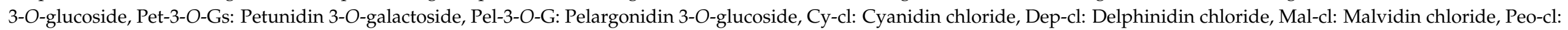
Peonidin chloride, and Pel-cl: pelargonidin chloride.

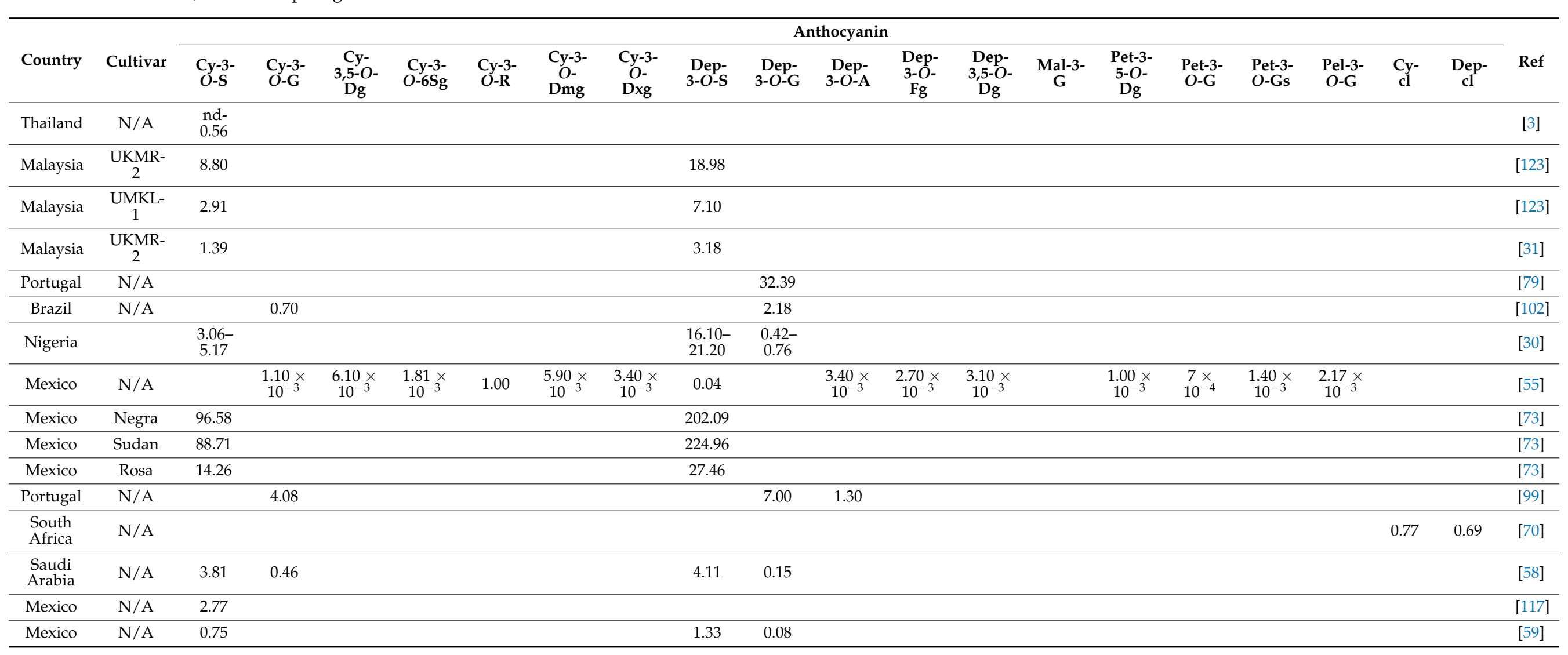


The content of anthocyanins in the roselle calyx will determine its color [98], and the genotype accounted for $60-80 \%$ of the variation in the calyx color [142]. Compared to roselles with a light-red color and those without red pigments (white cultivars), roselles with a dark-red calyx color had specific anthocyanin levels and higher antioxidant activity [39,108,127]. Specific anthocyanin compound levels were also found to be higher in certain environmental and postharvest conditions-namely, elevated $\mathrm{CO}_{2}$ environments [31], the dry season [30], and the drying process with a low temperature and low airflow velocity [46].

Individual anthocyanin stability against temperature, light, and the presence of other phenolic compounds has also been studied [33,70,71,101,157]. An increase in temperature made delphinidin 3-O-sambubioside more susceptible to degradation than cyanidin 3$O$-sambubioside [101]. One study observed that the anthocyanin retention was $87 \%$ at $50{ }^{\circ} \mathrm{C}$, then decreased to $61 \%$ at $80{ }^{\circ} \mathrm{C}$. The decrease in antioxidant activity began at $70{ }^{\circ} \mathrm{C}$ [70]. During light exposure, cyanidin-3-glucoside was reported to be completely degraded in four weeks, while it took seven weeks without light exposure [157]. Decreased rates of anthocyanin degradation to light exposure were reported in storage with low $\mathrm{pH}$ conditions [70]. Aimed to preserve the anthocyanin stability, several co-pigmentation studies were conducted using phenolic acids, such as gallic, ferulic, caffeic, and chlorogenic acids $[33,71]$. The addition of gallic acid was reported to retain anthocyanin stability. In contrast, an increase of ferulic and caffeic acids accelerated anthocyanin degradation [33].

\subsection{Organic Acid and Volatile Compounds}

The organic acid content, in the form of hibiscus acid and its derivatives, was reported to be best achieved in roselle extracts using the MAE method, with a total content of $70 \mathrm{mg} / \mathrm{g}$ [77]. Another study found hibiscus acid, hibiscus acid glucoside, and hibiscus acid 6-methyl ester, with a total range of hibiscus acid derivatives of 5.27-8.19 (mg/L), in dried and fresh roselle calyx extracts [53]. The lowest value was found in an extract of fresh calyx treated with high temperature, while the highest was in an extract of dried calyx treated with cold temperature. Other types of organic acids identified in roselle calyx include citric acid, malic acid, tartaric acid, ascorbic acid, hydroxycitric acid, hibiscus acid, and oxalic acid [16]. An infusion method reportedly produced extracts containing hydroxycitric acid that can potentially affect weight loss [39]. Other studies reported a citrate derivative roselle in methanol extract as a potential antihypertensive due to the ACE inhibitory activity [38]. The results of the isolation of hibiscus acid from the roselle study were reported at $13 \mathrm{~g} / \mathrm{kg}$ in the dried calyx and had the ability to inhibit pathogenic bacteria such as Escherichia coli and Salmonella [41].

The volatile content of roselle tissue was reported in several studies, both comparative studies between cultivars from different countries [104-108] and comparative studies between extraction methods [82,130]. Employing the GC-SPME (solid-phase microextraction) method, one study reported roselle with purple calyx had more major volatile compounds from fatty acid derivatives than roselle with red calyx [104]. From cultivar comparison studies, roselle with red calyx had a preferred aroma compound profile compared to a white calyx cultivar [108]. Sugar and fatty acid derivatives were reported as major volatile classes [104,107]. Other studies reported that geraniol is a major compound found from "Negra", "Rosa", "Blanca", and "Sudan" [106].

A study comparing the effects of extraction temperature and time to volatile compound contents reported that roselle extracted by a cold infusion method for $24 \mathrm{~h}$ resulted in higher total volatile compounds than those extracted by heat infusion for 16 or $40 \mathrm{~min}$. However, the identification of volatile compounds in all three beverages resulting from infusion showed the same types of compounds-namely, alcohols, furans, acids, ketones, aldehydes, volatile phenols, lactones, pyranone, pyrrole, terpene, and ester [130]. 


\section{Functional Properties}

\subsection{Antioxidant Activity}

Roselle is known as a plant that provides many health benefits-namely, as a source of antioxidants [14,15]. The antioxidant activity of roselle calyx has been confirmed in various studies $[92,129,155]$. Studies measuring the antioxidant activity of roselle calyx were conducted both through the study of extract characterizations $[66,102,115,121,158]$, comparisons between cultivars $[10,29,36,108,118,122]$ and between species $[34,90,92,114,115,119,137,139]$, calyx processing treatments $[125,147]$, and the comparison of extraction conditions $[3,28,58,64,65,81,82,99,111]$ and extraction optimization $[9,67,76]$. A comparison study of roselle to other edible flowers reported that orange roselle had a higher antioxidant activity and TPC than four other edible flowers [141], makes roselle become one of edible flower potential to be used in food development [159]. A similar result was reported by a study from Mediterranean region comparing the antioxidant activity of roselle to other herbs [35]. The high antioxidant activity of roselle might be due to its protocatechuic acid and gallic acid compounds [35]. The antioxidant capacity was also frequently attributed to the existence of phenolic acid and flavonoids [160]. Cyanidin-3-glucoside, delphinidin-3-sambubioside, delphinidin-3glucoside, and cyanidin-3-sambubioside from roselle extracts were reported to have high activity in 1-diphenyl-2-picrylhydrazyl (DPPH) inhibition [76]

Some methods frequently used in measuring the antioxidant activity of roselle calyx are the 1-diphenyl-2-picrylhydrazyl (DPPH) radical assay, ferric ion-reducing antioxidant power (FRAP) assay, and 2,2'-azino-bis-3-ethylbenzthiazoline-6-sulphonic acid (ABTS) assay. In addition, several studies have reported antioxidant activity by using the method of the oxygen radical absorbance capacity (ORAC) assay, ion-reducing capability assay, and ion-chelating assay. The most widely used method of measuring the antioxidant activity of roselle calyx was the DPPH assay $[3,9,10,28,29,31,34,36,57,58,64-67,70,74,76,81,82,90,92,95$, $102,111,114,115,118,119,121,122,125,129,137,139,145,147,155,158,161]$. The determination of the antioxidant activity was conducted through the measurement of free radical activity (diphenyl-picryl-hydrazyl) inhibition by antioxidant compounds via hydrogen atoms transfer and transforming the radicals into a nonradical compound (di-phenyl-picryl-hydrazine), which was characterized by a change in the mixture color from purple to yellow. Using the ABTS method, measurements of antioxidant activity are based on the interaction between the pre-generated ABTS radical cation with an antioxidant agent [162]. Antioxidant compounds will remove the color of the radical mono cation (ABTS-+). The use of the ABTS method for the measurement of the antioxidant activity of roselle calyx was reported in several studies $[57-59,73,92,98,102,116,117,119,125,127,129,147,150,155,161]$. Some studies used the FRAP method to determine the antioxidant properties of roselle calyx $[9,58,64,73,92,98,108,116,119,125,129,130,155,161]$. In the FRAP method, the antioxidant activity is measured through the ability of antioxidant compounds to reduce ferric tripyridyltriazine (Fe(III)TPTZ) to a ferro-tripyridyl-triazine complex (Fe(II)TPTZ).

The results using the DPPH method reported $92 \%$ scavenging activity in an acidifiedethanolic extract [66] and 97\% in an acidified-methanol extract [121]. A red calyx cultivar containing a high level of total anthocyanin also showed high antioxidant activity [115]. Nevertheless, a high antioxidant activity of roselle white calyx cultivar related to a high content of flavonoids and phenolic acids $[10,27,68,143]$. The white calyx cultivar "Alma Blanca" was reported to have the highest total phenolic acids, identified as caffeic and protocatechuic acid derivatives, compared to red calyx cultivars [118].

Similar to the TPC, TFC, and TAC, the antioxidant activity of roselle was influenced by environmental and processing conditions. Elevated ambient $\mathrm{CO}_{2}$ contents [31] and improving soil nutrients and physical properties [145] were reported to result in calyces with higher phenolic compound antioxidant activities. Furthermore, the extraction method used also influenced the antioxidant activity of the extract. Besides containing higher phenolic compounds, UAE was reported to extract a higher antioxidant activity than conventional maceration [10]. 


\subsection{Other Health Activities}

Roselle was famous as an ancient medicine due to its apparent efficacy [14]. In addition to having a high antioxidant activity, this section will discuss various studies of phenolic compound activities from roselles related to health benefits.

\subsubsection{Anti-Diabetes}

A meta-analysis study confirmed the benefits of roselle consumption as an antidiabetic [17]. Various studies were conducted to find out the potential of roselle phenolic compounds as an antidiabetic activity. The studies were conducted by measuring the inhibition of $\alpha$-amylase and $\alpha$-glucosidase $[30,57,87,109,113,114,163]$. $\alpha$-amylase and $\alpha$-glucosidase are the main enzymes in carbohydrate metabolism. The inhibition of both enzymes could potentially be included in diabetic therapy to lower high blood glucose levels. Several phenolic compounds were reported as having antidiabetic activity-namely, caffeic acid [114], gallic acid, and protocatechuic acids [113]. Another study reported that infusion drinks from calyx roselle containing rosmarinic acid also showed similar antidiabetic activity [57].

\subsubsection{Anti-Hyperlipidemia and Anti-Obesity}

The effects of roselle consumption in metabolic syndrome therapy, especially in lipid profile improvement, were also confirmed through various meta-analytical studies [17-19]. The reported mechanism was by lowering the levels of the total and LDL cholesterol. One study mentioned that the phenolic compound with anti-obesity potential was hydroxycitric acid [39]. The identification of phenolic compounds from roselle extract with the effect of decreasing the blood triglyceride levels of animals with a high-fat diet discovered 16 compounds, and further quantification showed that the dominant phenolic compounds were hibiscus acid, hydroxycitric acid, delphinidin 3-sambubioside, and cyanidin 3-sambubioside [116].

\subsubsection{Antihypertension}

Roselle was known in ancient medicine, because one of its reported health benefits was as an antihypertensive; several meta-analytical studies confirmed this benefit, with the results that roselle consumption decreased systolic blood pressure (SBP) and diastolic blood pressure (DBP) $[19,20]$. Several studies reported the antihypertension potential of phenolic compounds through the measurement of ACEI activity $[38,68]$. Some phenolic compounds reported having ACEI activity were ferulic acid, kaempferol glycosides, chlorogenate esters, and citrate derivatives [38].

\subsubsection{Diuretic}

In response to the ethnomedical use of roselle infusions and beverages, studies have confirmed the antidiuretic potential in the aqueous extract. These diuretic, as well as natriuretic, properties would augment the ACEI activity and support the antihypertensive and reno-protective action of roselle extract-namely, through vascular smooth muscle relaxation. The quercetin content in the extract was assumed to influence the vascular endothelium, resulting in NO release, and the inhibition of ATPase activity, affecting the $\mathrm{Na}+/ \mathrm{K}+$ concentration gradient in the tubular epithelial cells of the nephron [156].

Other health benefits of roselle calyx extract containing a phenolic compound are: (1) anti-urease: the roselle protocatechuic acid inhibitory properties against jack bean urease (JBU) and toxic effects on human gastric epithelial cells (GES-1) [126]; (2) anti-aging: the roselle calyx extract was evaluated for melanogenesis-stimulating activity [135] and inhibitory activity against collagenase, elastase, and hyaluronidase [129]; (3) neuroprotective: the roselle calyx extract was evaluated for the inhibition of cholinergic (cholinesterases), monoaminergic (monoamine oxidase), and purinergic (ecto-5 nucleotidase) enzyme activity [97]; (4) anticancer: the roselle extracts were evaluated for leukemia line K-562 activity [145] and against Caco-2, HepG-2, HCT8, and A549 cells [76]; (5) hematoprotective: 
the roselle ethanolic extract was evaluated for antigenotoxic and antimutagenic activity [10]; and (6) the antimicrobial: roselle extract was evaluated against Gram-negative (Escherichia coli and Salmonella enteritidis) and Gram-positive (Staphylococcus aureus and Micrococcus luteus) [122] bacteria; Listeria monocytogenes and methicillin-resistant Staphylococcus aureus (MRSA) [164]; and Bacillus subtilis (ATCC 6633), Staphylococcus aureus (ATCC 6538), and Escherichia coli (ATCC 8739) [82].

\section{Conclusions}

This systematic review presented alternative analytical methods for phenolic compound analyses in roselle (Hibiscus sabdariffa L.) from numerous studies reported from January 2010 to January 2021. The selection of the analytical methods, including extraction, separation, and detection, was dependent on the characteristics of the sample matrices and target analytes. The appropriate analytical methods supported the attempts to study various types of phenolic compounds in roselle with a wide variety of biological activities that are beneficial to human health. The major phenolic compounds naturally present in the red calyx cultivars were anthocyanins, i.e., cyanidin-3-sambubioside and delphinidin-3sambubioside; the white-calyx cultivars mostly contained flavonoids and phenolic acids. The contents of the phenolics in roselle are affected by cultivars, the production environment, and harvest and postharvest methods. Specific types of phenolic compounds provide particular advantages to human health. The compounds related to antidiabetic activity, for instance, were caffeic acid, gallic acid, and protocatechuic acid. In comparison, some other phenolic compounds exerted ACEI activity, such as are ferulic acid, kaempferol glycosides, and chlorogenate esters. The information discussed here could trigger further research in order to take greater advantage of roselle by assisting with the selection of analytical tools for the determination of beneficial compounds, as well as presenting the significant effects of these compounds on human health.

Author Contributions: Conceptualization, W.S.; methodology, W.S.; validation, W.S. and M.; formal analysis, B.W.H. and W.S.; investigation, B.W.H. and M.; writing-original draft preparation, B.W.H. and M.; writing - review and editing, W.S.; supervision, W.S. and M.; and funding acquisition, B.W.H. All authors have read and agreed to the published version of the manuscript.

Funding: This research was funded by The Ministry of Education and Culture of the Republic of Indonesia, through a SAINTEK study grant No. 3535-2018.

Institutional Review Board Statement: Not applicable.

Informed Consent Statement: Not applicable.

Data Availability Statement: Data is contained within the article.

Acknowledgments: This report formed part of the activity carried out by B.W.H. at the Department of Food and Agricultural Product Technology, Faculty of Agricultural Technology, Universitas Gadjah Mada.

Conflicts of Interest: The authors declare no conflict of interest.

\section{References}

1. Pires, T.C.S.P.; Barros, L.; Santos-Buelga, C.; Ferreira, I.C.F.R. Edible flowers: Emerging components in the diet. Trends Food Sci. Technol. 2019, 93, 244-258. [CrossRef]

2. Aziz, Z.; Wong, S.Y.; Chong, N.J. Effects of Hibiscus sabdariffa L. on serum lipids: A systematic review and meta-analysis. J. Ethnopharmacol. 2013, 150, 442-450. [CrossRef] [PubMed]

3. Ariyabukalakorn, V.; Panthong, S.; Itharat, A. Effects and chemical contents of hydrolysis modification of aqueous roselle extract to reflect the antioxidant and anti-inflammatory effects. Sci. Technol. Asia 2019, 24, 115-125. [CrossRef]

4. Zihad, S.M.N.K.; Gupt, Y.; Uddin, S.J.; Islam, M.T.; Alam, M.R.; Aziz, S.; Hossain, M.; Shilpi, J.A.; Nahar, L.; Sarker, S.D. Nutritional value, micronutrient and antioxidant capacity of some green leafy vegetables commonly used by southern coastal people of Bangladesh. Heliyon 2019, 5, e02768. [CrossRef] [PubMed]

5. Mojica, L.; Rui, L.; de Mejia, E.G. Hibiscus sabdariffa L.: Phytochemical Composition and Nutraceutical Properties. ACS Symp. Ser. 2012, 1109, 279-305. 
6. Pham, T.N.; Phu Nguyen, T.N.; Duc, L.T.; Nguyen, M.T.; Toan, T.Q.; Hong Nhan, L.T.; N-Vo, D.V.; Vo, T.S.; Bui, L.M. Response surface modeling and optimizing conditions for anthocyanins extraction from Hibiscussabdariffa L. (Roselle) grown in Lam Dong, Vietnam. IOP Conf. Ser. Mater. Sci. Eng. 2019, 544. [CrossRef]

7. Villani, T.; Juliani, H.R.; Simon, J.E.; Wu, Q. Hibiscus sabdariffa: Phytochemistry, Quality Control, and Health Properties. ACS Symp. Ser. 2013, 1127, 209-230.

8. Cassol, L.; Rodrigues, E.; Zapata Noreña, C.P. Extracting phenolic compounds from Hibiscus sabdariffa L. calyx using microwave assisted extraction. Ind. Crops Prod. 2019, 133, 168-177. [CrossRef]

9. Paraíso, C.M.; dos Santos, S.S.; Correa, V.G.; Magon, T.; Peralta, R.M.; Visentainer, J.V.; Madrona, G.S. Ultrasound assisted extraction of hibiscus (Hibiscus sabdariffa L.) bioactive compounds for application as potential functional ingredient. J. Food Sci. Technol. 2019, 56, 4667-4677. [CrossRef]

10. Pozos, G.I.P.; Ruiz-López, M.A.; Nátera, J.F.Z.; Moya, C.Á.; Ramírez, L.B.; Silva, M.R.; Macías, R.R.; García-López, P.M.; Cruz, R.G.; Pérez, E.S.; et al. Antioxidant capacity and antigenotoxic effect of Hibiscus sabdariffa L. extracts obtained with ultrasound-assisted extraction process. Appl. Sci. 2020, 10, 560. [CrossRef]

11. Alara, O.R.; Abdurahman, N.H. Microwave-assisted extraction of phenolics from Hibiscus sabdariffa calyces: Kinetic modelling and process intensification. Ind. Crops Prod. 2019, 137, 528-535. [CrossRef]

12. Pimentel-Moral, S.; Borrás-Linares, I.; Lozano-Sánchez, J.; Arráez-Román, D.; Martínez-Férez, A.; Segura-Carretero, A. Supercritical $\mathrm{CO}_{2}$ extraction of bioactive compounds from Hibiscus sabdariffa. J. Supercrit. Fluids 2019, 147, 213-221. [CrossRef]

13. Ojulari, O.V.; Lee, S.G.; Nam, J.O. Beneficial Effects of Natural Bioactive Compounds from Hibiscus sabdariffa L. On obesity. Molecules 2019, 24, 210. [CrossRef]

14. Riaz, G.; Chopra, R. A review on phytochemistry and therapeutic uses of Hibiscus sabdariffa L. Biomed. Pharmacother. 2018, 102, 575-586. [CrossRef]

15. Guardiola, S.; Mach, N. Therapeutic potential of Hibiscus sabdariffa: A review of the scientific evidence. Endocrinol. Nutr. 2014, 61, 274-295. [CrossRef] [PubMed]

16. Izquierdo-Vega, J.A.; Arteaga-Badillo, D.A.; Sánchez-Gutiérrez, M.; Morales-González, J.A.; Vargas-Mendoza, N.; Gómez-Aldapa, C.A.; Castro-Rosas, J.; Delgado-Olivares, L.; Madrigal-Bujaidar, E.; Madrigal-Santillán, E. Organic acids from Roselle (Hibiscus sabdariffa L.)-A brief review of its pharmacological effects. Biomedicines 2020, 8, 100. [CrossRef]

17. Bule, M.; Albelbeisi, A.H.; Nikfar, S.; Amini, M.; Abdollahi, M. The antidiabetic and antilipidemic effects of Hibiscus sabdariffa: A systematic review and meta-analysis of randomized clinical trials. Food Res. Int. 2020, 130, 108980. [CrossRef] [PubMed]

18. Zhang, B.; Yue, R.; Wang, Y.; Wang, L.; Chin, J.; Huang, X.; Jiang, Y. Effect of Hibiscus sabdariffa (Roselle) supplementation in regulating blood lipids among patients with metabolic syndrome and related disorders: A systematic review and meta-analysis. Phyther. Res. 2019, 1-13. [CrossRef] [PubMed]

19. Boushehri, S.N.; Karimbeiki, R.; Ghasempour, S.; Ghalishourani, S.S.; Pourmasoumi, M.; Hadi, A.; Mbabazi, M.; Pour, Z.K.; Assarroudi, M.; Mahmoodi, M.; et al. The efficacy of sour tea (Hibiscus sabdariffa L.) on selected cardiovascular disease risk factors: A systematic review and meta-analysis of randomized clinical trials. Phyther. Res. 2020, 34, 329-339. [CrossRef]

20. Serban, C.; Sahebkar, A.; Ursoniu, S.; Andrica, F.; Banach, M. Effect of sour tea (Hibiscus sabdariffa L.) on arterial hypertension: A systematic review and meta-analysis of randomized controlled trials. J. Hypertens. 2015, 33, 1119-1127. [CrossRef] [PubMed]

21. Cid-Ortega, S.; Guerrero-Beltrán, J.A. Roselle calyces (Hibiscus sabdariffa), an alternative to the food and beverages industries: A review. J. Food Sci. Technol. 2015, 52, 6859-6869. [CrossRef]

22. Purbowati, I.S.M.; Maksum, A. The antioxidant activity of Roselle (Hibiscus sabdariffa Linii) phenolic compounds in different variations microwave-Assisted extraction time and power. IOP Conf. Ser. Earth Environ. Sci. 2019, 406. [CrossRef]

23. Da-Costa-Rocha, I.; Bonnlaender, B.; Sievers, H.; Pischel, I.; Heinrich, M. Hibiscus sabdariffa L.-A phytochemical and pharmacological review. Food Chem. 2014, 165, 424-443. [CrossRef]

24. Nadlene, R.; Sapuan, S.M.; Jawaid, M.; Ishak, M.R.; Yusriah, L. A Review on Roselle Fiber and Its Composites. J. Nat. Fibers 2016, 13, 10-41. [CrossRef]

25. Patel, S. Hibiscus sabdariffa: An ideal yet under-exploited candidate for nutraceutical applications. Biomed. Prev. Nutr. 2014, 4, 23-27. [CrossRef]

26. Qi, Y.; Chin, K.L.; Malekian, F.; Berhane, M.; Gager, J. Biological Characteristics, Nutritional and Medicinal Value of Roselle, Hibiscus sabdariffa Biological Characteristics, Nutritional and Medicinal Value of Roselle, Hibiscus sabdariffa. Agric. Res. Ext. Cent. 2005, 70813, 603-604.

27. Tahir, H.E.; Arslan, M.; Mahunu, G.K.; Mariod, A.A.; Wen, Z.; Xiaobo, Z.; Xiaowei, H.; Jiyong, S.; El-Seedi, H. Authentication of the geographical origin of Roselle (Hibiscus sabdariffa L) using various spectroscopies: NIR, low-field NMR and fluorescence. Food Control. 2020, 114, 107231. [CrossRef]

28. Jabeur, I.; Pereira, E.; Caleja, C.; Calhelha, R.C.; Soković, M.; Catarino, L.; Barros, L.; Ferreira, I.C.F.R. Exploring the chemical and bioactive properties of: Hibiscus sabdariffa L. calyces from Guinea-Bissau (West Africa). Food Funct. 2019, 10, 2234-2243. [CrossRef]

29. Hinojosa-Gómez, J.; Martin-Hernández, C.S.; Heredia, J.B.; León-Félix, J.; Osuna-Enciso, T.; Muy-Rangel, M.D. Roselle (Hibiscus sabdariffa L.) cultivars calyx produced hydroponically: Physicochemical and nutritional quality. Chil. J. Agric. Res. 2018, 78, 478-485. [CrossRef]

30. Ifie, I.; Ifie, B.E.; Ibitoye, D.O.; Marshall, L.J.; Williamson, G. Seasonal variation in Hibiscus sabdariffa (Roselle) calyx phytochemical profile, soluble solids and $\alpha$-glucosidase inhibition. Food Chem. 2018, 261, 164-168. [CrossRef] 
31. Ali, S.A.M.; Zain, C.R.C.M.; Latip, J. Influence of elevated $\mathrm{CO}_{2}$ on the growth and phenolic constituents. J. Teknol. 2019, 3, 109-118.

32. Alara, O.R.; Abdurahman, N.H.; Obanijesu, E.O.; Alara, J.A.; Abdul Mudalip, S.K. Extract-rich in flavonoids from Hibiscus sabdariffa calyces: Optimizing microwave-assisted extraction method and characterization through LC-Q-TOF-MS analysis. J. Food Process. Eng. 2019, 43. [CrossRef]

33. Zhang, Y.; Sang, J.; Chen, F.F.; Sang, J.; Li, C. qin $\beta$-Cyclodextrin-assisted extraction and green chromatographic analysis of Hibiscus sabdariffa L. anthocyanins and the effects of gallic/ferulic/caffeic acids on their stability in beverages. J. Food Meas. Charact. 2018, 12, 2475-2483. [CrossRef]

34. Samadi, S.; Fard, F.R. Phytochemical properties, antioxidant activity and mineral content (Fe, Zn and Cu) in Iranian produced black tea, green tea and roselle calyces. Biocatal. Agric. Biotechnol. 2020, 23, 101472. [CrossRef]

35. Rababah, T.M.; Ereifej, K.I.; Esoh, R.B.; Al-U’Datt, M.H.; Alrababah, M.A.; Yang, W. Antioxidant activities, total phenolics and HPLC analyses of the phenolic compounds of extracts from common Mediterranean plants. Nat. Prod. Res. 2011, 25, 596-605. [CrossRef]

36. Özdogan, F.P.; Orhan, N.; Ergun, F. Studies on the conformity of Hibiscus sabdariffa L. samples from turkish market to european pharmacopeia. Fabad J. Pharm. Sci. 2011, 36, 25-32.

37. Jacob, J.P.S.; Shenbagaraman, S. Evaluation of antioxidant and antimicrobial activities of the selected green leafy vegetables. Int. J. PharmTech Res. 2011, 3, 148-152.

38. Salem, M.A.; Michel, H.E.; Ezzat, M.I.; Okba, M.M.; El-desoky, A.M.; Mohamed, S.O.; Ezzat, S.M. Optimization of an Extraction Solvent for Angiotensin-Converting Enzyme Inhibitors from Hibiscus sabdariffa L. Based on Its UPLC-MS/MS Metabolic Profiling. Molecules 2020, 25, 2307. [CrossRef] [PubMed]

39. Rasheed, D.M.; Porzel, A.; Frolov, A.; El Seedi, H.R.; Wessjohann, L.A.; Farag, M.A. Comparative analysis of Hibiscus sabdariffa (roselle) hot and cold extracts in respect to their potential for $\alpha$-glucosidase inhibition. Food Chem. 2018, 250, 236-244. [CrossRef]

40. Gulsheen; Kumar, A.; Sharma, A. Antianxiety and Antidepressant Activity Guided Isolation and Characterization of Gossypetin from Hibiscus sabdariffa Linn. Calyces. J. Biol. Act. Prod. Nat. 2019, 9, 205-214. [CrossRef]

41. Portillo-Torres, L.A.; Bernardino-Nicanor, A.; Gómez-Aldapa, C.A.; González-Montiel, S.; Rangel-Vargas, E.; Villagómez-Ibarra, J.R.; González-Cruz, L.; Cortés-López, H.; Castro-Rosas, J. Hibiscus acid and chromatographic fractions from Hibiscus sabdariffa calyces: Antimicrobial activity against multidrug-resistant pathogenic bacteria. Antibiotics 2019, 8, 218. [CrossRef]

42. Wilson, F.D.; Menzel, M.Y. Kenaf (Hibiscus cannabinus), Roselle (Hibiscus sabdariffa). Econ. Bot. 1964, 18, 80-91. [CrossRef]

43. Hopkins, A.L.; Lamm, M.G.; Funk, J.; Ritenbaugh, C. Hibiscus sabdariffa L. in the treatment of hypertension and hyperlipidemia: A comprehensive review of animal and human studies. Fitoterapia 2013, 85, 84-94. [CrossRef] [PubMed]

44. Adeola, O.; Batista, C.M.D.; Fungwe, T.V. The Effectiveness of Hibiscus sabdariffa for Improving Metabolic Syndrome: A Systematic Review. EC Nutr. 2019, 10, 887-892.

45. Kafkas, N.E.; Kosar, M.; Öz, A.T.; Mitchell, A.E. Advanced Analytical Methods for Phenolics in Fruits. J. Food Qual. 2018, 2018. [CrossRef]

46. Tham, T.C.; Ng, M.X.; Gan, S.H.; Chua, L.S.; Aziz, R.; Abdullah, L.C.; Ong, S.P.; Chin, N.L.; Law, C.L. Impacts of different drying strategies on drying characteristics, the retention of bio-active ingredient and colour changes of dried Roselle. Chin. J. Chem. Eng. 2018, 26, 303-316. [CrossRef]

47. Belwal, T.; Ezzat, S.M.; Rastrelli, L.; Bhatt, I.D.; Daglia, M.; Baldi, A.; Devkota, H.P.; Orhan, I.E.; Patra, J.K.; Das, G.; et al. A critical analysis of extraction techniques used for botanicals: Trends, priorities, industrial uses and optimization strategies. TrAC Trends Anal. Chem. 2018, 100, 82-102. [CrossRef]

48. Lefebvre, T.; Destandau, E.; Lesellier, E. Selective extraction of bioactive compounds from plants using recent extraction techniques: A review. J. Chromatogr. A 2021, 1635, 461770. [CrossRef]

49. Ignat, I.; Volf, I.; Popa, V.I. Natural Products: Phytochemistry, Botany and Metabolism of Alkaloids, Phenolics and Terpenes; Springer: Berlin/Heidelberg, Germany, 2013; ISBN 9783642221446.

50. Setyaningsih, W.; Saputro, I.E.; Palma, M.; Barroso, C.G. Optimization of the ultrasound-assisted extraction of tryptophan and its derivatives from rice (Oryza sativa) grains through a response surface methodology. J. Cereal Sci. 2017, 75, 192-197. [CrossRef]

51. Alam, G.; Sartini; Alfath, A. Comparison of microwave assisted extraction (MAE) with variations of power and infusion extraction method on antibacterial activity of rosella calyx extract (Hibiscus sabdariffa). J. Phys. Conf. Ser. 2019, 1341. [CrossRef]

52. Ochoa-Velasco, C.E.; Ruiz-López, I.I. Mass transfer modeling of the antioxidant extraction of roselle flower (Hibiscus sabdariffa). J. Food Sci. Technol. 2019, 56, 1008-1015. [CrossRef] [PubMed]

53. Ramírez-Rodrigues, M.M.; Balaban, M.O.; Marshall, M.R.; Rouseff, R.L. Hot and Cold Water Infusion Aroma Profilesof Hibiscus sabdariffa: Fresh Compared with Dried. J. Food Sci. 2011, 76, 212-217. [CrossRef] [PubMed]

54. Aryanti, N.; Nafiunisa, A.; Wardhani, D.H. Conventional and ultrasound-assisted extraction of anthocyanin from red and purple roselle (Hibiscus sabdariffa L.) calyces and characterisation of its anthocyanin powder. Int. Food Res. J. 2019, 26, 529-535.

55. Amaya-Cruz, D.M.; Perez-Ramirez, I.F.; Ortega-Diaz, D.; Rodriguez-Garcia, M.E.; Reynoso-Camacho, R. Roselle (Hibiscus sabdariffa) by-product as functional ingredient: Effect of thermal processing and particle size reduction on bioactive constituents and functional, morphological, and structural properties. J. Food Meas. Charact. 2018, 12, 135-144. [CrossRef]

56. Aryanti, N.; Nafiunisa, A.; Wardhani, D.H.; Kumoro, A.C. Extraction characteristic and microencapsulation of anthocyanin as natural food colouring from roselle calyces by ultrasound-assisted extraction. J. Bahan Alam Terbarukan 2017, 6, 87-96. [CrossRef] 
57. Pérez-Ramírez, I.F.; Castaño-Tostado, E.; Ramírez-De León, J.A.; Rocha-Guzmán, N.E.; Reynoso-Camacho, R. Effect of stevia and citric acid on the stability of phenolic compounds and in vitro antioxidant and antidiabetic capacity of a roselle (Hibiscus sabdariffa L.) beverage. Food Chem. 2015, 172, 885-892. [CrossRef]

58. Sindi, H.A.; Marshall, L.J.; Morgan, M.R.A. Comparative chemical and biochemical analysis of extracts of Hibiscus sabdariffa. Food Chem. 2014, 164, 23-29. [CrossRef]

59. Salazar-González, C.; Vergara-Balderas, F.T.; Ortega-Regules, A.E.; -Beltrán, J.Á. Antioxidant properties and color of Hibiscus sabdariffa extracts. Cienc. Investig. Agrar. 2012, 39, 79-90. [CrossRef]

60. Cissé, M.; Bohuon, P.; Sambe, F.; Kane, C.; Sakho, M.; Dornier, M. Aqueous extraction of anthocyanins from Hibiscus sabdariffa: Experimental kinetics and modeling. J. Food Eng. 2012, 109, 16-21. [CrossRef]

61. Cisse, M.; Vaillant, F.; Soro, D.; Reynes, M.; Dornier, M. Crossflow microfiltration for the cold stabilization of roselle (Hibiscus sabdariffa L.) extract. J. Food Eng. 2011, 106, 20-27. [CrossRef]

62. Pham, T.N.; Nguyen, T.N.P.; Lam, T.D.; Tran, T.H.; Nguyen, D.C.; Vo, D.V.N.; Le, X.T.; Do, S.T.; Bach, L.G. Effects of various solvent concentration, liquid-solid ratio, temperatures and time values on the extraction yield of anthocyanin from Vietnam Hibiscus sabdariffa L. (Roselle). IOP Conf. Ser. Mater. Sci. Eng. 2019, 542. [CrossRef]

63. Bergmeier, D.; Berres, P.H.D.; Filippi, D.; Bilibio, D.; Bettiol, V.R.; Priamo, W.L. Extraction of total polyphenols from hibiscus (Hibiscus sabdariffa L.) and waxweed/'sete-sangrias' (Cuphea carthagenensis) and evaluation of their antioxidant potential. Acta Sci. Technol. 2014, 36, 545-551. [CrossRef]

64. Duy, N.Q.; Thoai, H.; Lam, T. Effects of Different Extraction Solvent Systems on Total Phenolic, Total Flavonoid, Total Anthocyanin Contents and Antioxidant Activities of Roselle (Hibiscus sabdariffa L.) Extracts. ASIAN J. Chem. 2019, 31, 2517-2521. [CrossRef]

65. Anokwuru, C.P.; Esiaba, J.; Ajibaye, O.; Adesuyi, A.O. Polyphenolic content and antioxidant activity of Hibiscus sabdariffa calyx. Res. J. Med. Plant. 2011, 5, 557-566. [CrossRef]

66. Ajiboye, T.O.; Salawu, N.A.; Yakubu, M.T.; Oladiji, A.T.; Akanji, M.A.; Okogun, J.I. Antioxidant and drug detoxification potentials of Hibiscus sabdariffa anthocyanin extract. Drug Chem. Toxicol. 2011, 34, 109-115. [CrossRef]

67. Kurtulbaş, E.; Pekel, A.G.; Bilgin, M.; Makris, D.P.; Şahin, S. Citric acid-based deep eutectic solvent for the anthocyanin recovery from Hibiscus sabdariffa through microwave-assisted extraction. Biomass Convers. Biorefinery 2020. [CrossRef]

68. Vargas-León, E.A.; Díaz-Batalla, L.; González-Cruz, L.; Bernardino-Nicanor, A.; Castro-Rosas, J.; Reynoso-Camacho, R.; Gómez-Aldapa, C.A. Effects of acid hydrolysis on the free radical scavenging capacity and inhibitory activity of the angiotensin converting enzyme of phenolic compounds of two varieties of jamaica (Hibiscus sabdariffa). Ind. Crops Prod. 2018, 116, 201-208. [CrossRef]

69. Aryanti, N.; Nafiunisa, A.; Bella, N.; Sanjaya, R.; Wardhani, D.H.; Kumoro, A.C. Kinetics of ultrasound-assisted extraction of anthocyanin from purple roselle calyces under different PH conditions. Chem. Chem. Technol. 2018, 12, 523-528. [CrossRef]

70. Sipahli, S.; Mohanlall, V.; Mellem, J.J. Stability and degradation kinetics of crude anthocyanin extracts from H. sabdariffa. Food Sci. Technol. 2017, 37, 209-215. [CrossRef]

71. Grajeda-Iglesias, C.; Figueroa-Espinoza, M.C.; Barouh, N.; Baréa, B.; Fernandes, A.; De Freitas, V.; Salas, E. Isolation and Characterization of Anthocyanins from Hibiscus sabdariffa Flowers. J. Nat. Prod. 2016, 79, 1709-1718. [CrossRef]

72. Deli, M.; Ndjantou, E.B.; Ngatchic Metsagang, J.T.; Petit, J.; Njintang Yanou, N.; Scher, J. Successive grinding and sieving as a new tool to fractionate polyphenols and antioxidants of plants powders: Application to Boscia senegalensis seeds, Dichrostachys glomerata fruits, and Hibiscus sabdariffa calyx powders. Food Sci. Nutr. 2019, 7, 1795-1806. [CrossRef]

73. Camelo-Méndez, G.A.; Vanegas-Espinoza, P.E.; Escudero-Gilete, M.L.; Heredia, F.J.; Paredes-López, O.; Del Villar-Martínez, A.A. Colorimetric Analysis of Hibiscus Beverages and their Potential Antioxidant Properties. Plant. Foods Hum. Nutr. 2018, 73, $247-252$. [CrossRef] [PubMed]

74. Duy, N.Q.; Pham, T.N.; Binh, M.L.T.; Thuan, M.; Van, N.T.T.; Lam, T.D.; Nhan, P.N.T. Effects of extraction conditions on antioxidant activities of roselle (Hibiscus sabdariffa L.) extracts. Mater. Sci. Forum 2020, 977, 201-206. [CrossRef]

75. Duy, N.Q.; Binh, M.L.T.; Thuan, M.; Van, N.T.T.; Lam, T.D.; Tran, T.H.; Nhan, P.N.T. Effects of extraction conditions on total phenolic content and total flavonoid content of roselle (Hibiscus sabdariffa L.) extracts. Key Eng. Mater. 2019, 814, 469-474. [CrossRef]

76. Maciel, L.G.; do Carmo, M.A.V.; Azevedo, L.; Daguer, H.; Molognoni, L.; de Almeida, M.M.; Granato, D.; Rosso, N.D. Hibiscus sabdariffa anthocyanins-rich extract: Chemical stability, in vitro antioxidant and antiproliferative activities. Food Chem. Toxicol. 2018, 113, 187-197. [CrossRef]

77. Pimentel-Moral, S.; Borrás-Linares, I.; Lozano-Sánchez, J.; Arráez-Román, D.; Martínez-Férez, A.; Segura-Carretero, A. Microwaveassisted extraction for Hibiscus sabdariffa bioactive compounds. J. Pharm. Biomed. Anal. 2018, 156, 313-322. [CrossRef]

78. Miranda-Medina, A.; Hayward-Jones, P.M.; Carvajal-Zarrabal, O.; de Guevara-Vela, L.d.A.L.; Ramírez-Villagómez, Y.D.; Barradas-Dermitz, D.M.; Luna-Carrillo, G.; Aguilar-Uscanga, M.G. Optimization of Hibiscus sabdariffa L. (Roselle) anthocyanin aqueous-ethanol extraction parameters using response surface methodology. Sci. Study Res. Chem. Chem. Eng. Biotechnol. Food Ind. 2018, 19, 53-62.

79. Pinela, J.; Prieto, M.A.; Pereira, E.; Jabeur, I.; Barreiro, M.F.; Barros, L.; Ferreira, I.C.F.R. Optimization of heat- and ultrasoundassisted extraction of anthocyanins from Hibiscus sabdariffa calyces for natural food colorants. Food Chem. 2019, 275, 309-321. [CrossRef] 
80. Lukmanto, S.; Roesdiyono, N.; Ju, Y.H.; Indraswati, N.; Soetaredjo, F.E.; Ismadji, S. Supercritical Co2 Extraction of Phenolic Compounds in Roselle(Hibiscus sabdariffa L.). Chem. Eng. Commun. 2013, 200, 1187-1196. [CrossRef]

81. Lourith, N.; Kanlayavattanakul, M. Antioxidant activity and stability of natural colour recovered from Roselle juice production. Agro Food Ind. Hi Tech. 2013, 24, 40-43.

82. Jung, E.; Kim, Y.; Joo, N. Physicochemical properties and antimicrobial activity of Roselle (Hibiscus sabdariffa L.). J. Sci. Food Agric. 2013, 93, 3769-3776. [CrossRef] [PubMed]

83. Ramirez-Rodrigues, M.M.; Plaza, M.L.; Azeredo, A.; Balaban, M.O.; Marshall, M.R. Physicochemical and phytochemical properties of cold and hot water extraction from Hibiscus sabdariffa. J. Food Sci. 2011, 76, C428-C435. [CrossRef]

84. Capuzzo, A.; Maffei, M.E.; Occhipinti, A. Supercritical fluid extraction of plant flavors and fragrances. Molecules 2013, 18, 7194-7238. [CrossRef]

85. Idham, Z.; Nasir, H.M.; Yunus, M.A.C.; Yian, L.N.; Peng, W.L.; Hassan, H.; Setapar, S.H.M. Optimisation of supercritical CO 2 extraction of red colour from roselle (Hibiscus sabdariffa Linn.) calyces. Chem. Eng. Trans. 2017, 56, 871-876. [CrossRef]

86. Opletal, L.; Chocholousova-Havlikova, L.; Siatka, T.; Cahliková, L.; Locarek, M.; Ali, B.H.; Manoj, P.; Ramkumar, A.; Al Suleimani, Y.M.; Al Za'Abi, M.; et al. Preparation and validated analysis of anthocyanin concentrate from the calyces of Hibiscus sabdariffa. Nat. Prod. Commun. 2017, 12, 44-45. [CrossRef]

87. Xu, Y.; Hu, D.; Bao, T.; Xie, J.; Chen, W. A simple and rapid method for the preparation of pure delphinidin-3-O-sambubioside from Roselle and its antioxidant and hypoglycemic activity. J. Funct. Foods 2017, 39, 9-17. [CrossRef]

88. Grajeda-Iglesias, C.; Salas, E.; Barouh, N.; Baréa, B.; Figueroa-Espinoza, M.C. Lipophilization and MS characterization of the main anthocyanins purified from hibiscus flowers. Food Chem. 2017, 230, 189-194. [CrossRef] [PubMed]

89. Cisse, M.; Vaillant, F.; Kane, A.; Ndiaye, O.; Dornier, M. Impact of the extraction procedure on the kinetics of anthocyanin and colour degradation of roselle extracts during storage. J. Sci. Food Agric. 2012, 92, 1214-1221. [CrossRef]

90. Trinh, L.T.P.; Choi, Y.S.; Bae, H.J. Production of phenolic compounds and biosugars from flower resources via several extraction processes. Ind. Crops Prod. 2018, 125, 261-268. [CrossRef]

91. Amlashi, H.A.; Madani, H.; Sonboli, A.; Khaghani, S.; Ramezani, M. Volatile composition of the leaves and calyces essential oil of roselle (Hibiscus sabdariffa L.) from Iran. J. Essent. Oil Bear. Plants 2020, 23, 743-755. [CrossRef]

92. Pengkumsri, N.; Kaewdoo, K.; Leeprechanon, W.; Sivamaruthi, B.S. Influence of extraction methods on total phenolic content and antioxidant properties of some of the commonly used plants in Thailand. Pakistan J. Biol. Sci. 2019, 22, 117-126. [CrossRef]

93. Ramírez-Cortés, B.; de Caro-Velarde, F.J.; Valdivia-Reynoso, M.G.; Ramírez-Lozano, M.H.; Machuca-Sánchez, L. Changes in size and chemical characteristics of roselle (Hibiscus sabdariffa L.) calyces during maturation. Rev. Chapingo Ser. Hortic. 2011, XVII, 19-31.

94. Pragalyaashree, M.M.; Tiroutchelvame, D.; Sashikumar, S. Degradation kinetics of anthocyanin extracted from roselle calyces (Hibiscus sabdariffa). J. Appl. Pharm. Sci. 2018, 8, 57-63. [CrossRef]

95. Inggrid, H.M.; Jaka; Santoso, H. Natural red dyes extraction on roselle petals. IOP Conf. Ser. Mater. Sci. Eng. 2016, 162. [CrossRef]

96. Tavakolifar, F.; Givianrad, M.H.; Saber-Tehrani, M. Extraction of anthocyanins from Hibiscus sabdariffa and assessment of its antioxidant properties in extra virgin olive oil. Fresenius Environ. Bull. 2016, 25, 3709-3713.

97. Oboh, G.; Adewuni, T.M.; Ademiluyi, A.O.; Olasehinde, T.A.; Ademosun, A.O. Phenolic Constituents and Inhibitory Effects of Hibiscus sabdariffa L. (Sorrel) Calyx on Cholinergic, Monoaminergic, and Purinergic Enzyme Activities. J. Diet. Suppl. 2018, 15, 910-922. [CrossRef] [PubMed]

98. Camelo-Méndez, G.A.; Jara-Palacios, M.J.; Escudero-Gilete, M.L.; Gordillo, B.; Hernanz, D.; Paredes-López, O.; Vanegas-Espinoza, P.E.; Del Villar-Martínez, A.A.; Heredia, F.J. Comparative Study of Phenolic Profile, Antioxidant Capacity, and Color-composition Relation of Roselle Cultivars with Contrasting Pigmentation. Plant. Foods Hum. Nutr. 2016, 71, 109-114. [CrossRef] [PubMed]

99. Jabeur, I.; Pereira, E.; Barros, L.; Calhelha, R.C.; Soković, M.; Oliveira, M.B.P.P.; Ferreira, I.C.F.R. Hibiscus sabdariffa L. as a source of nutrients, bioactive compounds and colouring agents. Food Res. Int. 2017, 100, 717-723. [CrossRef] [PubMed]

100. Sinela, A.M.; Mertz, C.; Achir, N.; Rawat, N.; Vidot, K.; Fulcrand, H.; Dornier, M. Exploration of reaction mechanisms of anthocyanin degradation in a roselle extract through kinetic studies on formulated model media. Food Chem. 2017, 235, 67-75. [CrossRef]

101. Sinela, A.; Rawat, N.; Mertz, C.; Achir, N.; Fulcrand, H.; Dornier, M. Anthocyanins degradation during storage of Hibiscus sabdariffa extract and evolution of its degradation products. Food Chem. 2017, 214, 234-241. [CrossRef]

102. Piovesana, A.; Rodrigues, E.; Noreña, C.P.Z. Composition analysis of carotenoids and phenolic compounds and antioxidant activity from hibiscus calyces (Hibiscus sabdariffa L.) by HPLC-DAD-MS/MS. Phytochem. Anal. 2019, 30, 208-217. [CrossRef]

103. Juhari, N.H.; Bredie, W.L.P.; Toldam-Andersen, T.B.; Petersen, M.A. Characterization of Roselle calyx from different geographical origins. Food Res. Int. 2018, 112, 378-389. [CrossRef] [PubMed]

104. Ningrum, A.; Schreiner, M.; Luna, P.; Khoerunnisa, F.; Tienkink, E. Free volatile compounds in red and purple roselle (Hibiscus sabdariffa) pomace from Indonesia. Food Res. 2019, 3, 749-754. [CrossRef]

105. Avalos-Martínez, E.; Pino, J.A.; Sáyago-Ayerdi, S.; Sosa-Moguel, O.; Cuevas-Glory, L. Assessment of volatile compounds and sensory characteristics of Mexican hibiscus (Hibiscus sabdariffa L.) calyces hot beverages. J. Food Sci. Technol. 2019, 56, 360-366. [CrossRef] 
106. Camelo-Méndez, G.A.; Ragazzo-Sánchez, J.A.; Jiménez-Aparicio, A.R.; Vanegas-Espinoza, P.E.; Paredes-López, O.; Del Villar-Martínez, A.A. Comparative Study of Anthocyanin and Volatile Compounds Content of Four Varieties of Mexican Roselle (Hibiscus sabdariffa L.) by Multivariable Analysis. Plant. Foods Hum. Nutr. 2013, 68, 229-234. [CrossRef] [PubMed]

107. Farag, M.A.; Rasheed, D.M.; Kamal, I.M. Volatiles and primary metabolites profiling in two Hibiscus sabdariffa (roselle) cultivars via headspace SPME-GC-MS and chemometrics. Food Res. Int. 2015, 78, 327-335. [CrossRef] [PubMed]

108. Tahir, H.E.; Xiaobo, Z.; Mariod, A.A.; Mahunu, G.K.; Abdualrahman, M.A.Y.; Tchabo, W. Assessment of antioxidant properties, instrumental and sensory aroma profile of red and white Karkade/Roselle (Hibiscus sabdariffa L.). J. Food Meas. Charact. 2017, 11, 1559-1568. [CrossRef]

109. Ifie, I.; Abrankó, L.; Villa-Rodriguez, J.A.; Papp, N.; Ho, P.; Williamson, G.; Marshall, L.J. The effect of ageing temperature on the physicochemical properties, phytochemical profile and $\alpha$-glucosidase inhibition of Hibiscus sabdariffa (roselle) wine. Food Chem. 2018, 267, 263-270. [CrossRef]

110. Khoddami, A.; Wilkes, M.A.; Roberts, T.H. Techniques for analysis of plant phenolic compounds. Molecules 2013, 18, 2328-2375. [CrossRef] [PubMed]

111. Fitrotunnisa, Q.; Arsianti, A.; Tejaputri, N.A.; Qorina, F. Antioxidative activity and phytochemistry profile of Hibiscus sabdariffa herb extracts. Int. J. Appl. Pharm. 2019, 11, 29-32. [CrossRef]

112. Builders, P.; Kabele-Toge, B.; Builders, M.; Chindo, B.; Anwunobi, P.; Isimi, Y. Wound healing potential of formulated extract from Hibiscus sabdariffa calyx. Indian J. Pharm. Sci. 2013, 75, 45-52. [CrossRef]

113. Alegbe, E.O.; Teralı, K.; Olofinsan, K.A.; Surgun, S.; Ogbaga, C.C.; Ajiboye, T.O. Antidiabetic activity-guided isolation of gallic and protocatechuic acids from Hibiscus sabdariffa calyxes. J. Food Biochem. 2019, 43, 1-12. [CrossRef]

114. Wongsa, P.; Chaiwarit, J.; Zamaludien, A. In vitro screening of phenolic compounds, potential inhibition against $\alpha$-amylase and $\alpha$-glucosidase of culinary herbs in Thailand. Food Chem. 2012, 131, 964-971. [CrossRef]

115. Sarkar, B.; Vyas, P.; Haque, I.; Mukhopadhyay, K. A rapid UPLC method for simultaneous separation and detection of anthocyanidins from Ocimum, Hibiscus and Syzygium species and estimation of their antioxidant activity. J. Liq. Chromatogr. Relat. Technol. 2018, 41, 658-667. [CrossRef]

116. Fernández-Arroyo, S.; Rodríguez-Medina, I.C.; Beltrán-Debón, R.; Pasini, F.; Joven, J.; Micol, V.; Segura-Carretero, A.; FernándezGutiérrez, A. Quantification of the polyphenolic fraction and in vitro antioxidant and in vivo anti-hyperlipemic activities of Hibiscus sabdariffa aqueous extract. Food Res. Int. 2011, 44, 1490-1495. [CrossRef]

117. Daniel, D.L.; Huerta, B.E.B.; Sosa, I.A.; Mendoza, M.G.V. Effect of fixed bed drying on the retention of phenolic compounds, anthocyanins and antioxidant activity of roselle (Hibiscus sabdariffa L.). Ind. Crops Prod. 2012, 40, 268-276. [CrossRef]

118. Reyes-Luengas, A.; Salinas-Moreno, Y.; Ovando-Cruz, M.E.; Arteaga-Garibay, R.I.; Martínez-Peña, M.D. Análisis de ácidos fenólicos y actividad antioxidante de extractos acuosos de variedades de Jamaica (Hibiscus sabdariffa L.) con cálices de colores diversos analysis of phenolic acids and antioxidant activity of aqueous. Agrociencia 2015, 49, 277-290.

119. Chaiyasut, C.; Sivamaruthi, B.S.; Pengkumsri, N.; Sirilun, S.; Peerajan, S.; Chaiyasut, K.; Kesika, P. Anthocyanin profile and its antioxidant activity of widely used fruits, vegetables, and flowers in Thailand. Asian J. Pharm. Clin. Res. 2016, 9, 218-224. [CrossRef]

120. Achir, N.; Sinela, A.; Mertz, C.; Fulcrand, H.; Dornier, M. Monitoring anthocyanin degradation in Hibiscus sabdariffa extracts with multi-curve resolution on spectral measurement during storage. Food Chem. 2019, 271, 536-542. [CrossRef]

121. Obouayeba, A.P.; Djyh, N.B.; Sekou, D.; Djaman, A.J.; N'guessan, J.D.; Kone, M.; Kouakou, T.H. Phytochemical and Antioxidant Activity of Roselle (Hibiscus sabdariffa L.) Petal Extracts. Res. J. Pharm. Biol. Chem. Sci. 2013, 4, 1694-1720.

122. Borrás-Linares, I.; Fernández-Arroyo, S.; Arráez-Roman, D.; Palmeros-Suárez, P.A.; Del Val-Díaz, R.; Andrade-Gonzáles, I.; Fernández-Gutiérrez, A.; Gómez-Leyva, J.F.; Segura-Carretero, A. Characterization of phenolic compounds, anthocyanidin, antioxidant and antimicrobial activity of 25 varieties of Mexican Roselle (Hibiscus sabdariffa). Ind. Crops Prod. 2015, 69, 385-394. [CrossRef]

123. Ali, S.A.M.; Zainalabidin, S.; Latip, J. Quantitative analysis of phenolics content in two roselle varieties (Hibiscus sabdariffa) by high performance liquid chromatography. Malaysian J. Anal. Sci. 2019, 23, 715-724. [CrossRef]

124. Ali, S.A.M.; Mohd, C.R.C.; Latip, J. Comparison of phenolic constituent in Hibiscus sabdariffa cv. UKMR-2 calyx at different harvesting times. Sains Malaysiana 2019, 48, 1417-1424. [CrossRef]

125. Mercado-Mercado, G.; Blancas-Benitez, F.J.; Velderrain-Rodríguez, G.R.; Montalvo-González, E.; González-Aguilar, G.A.; AlvarezParrilla, E.; Sáyago-Ayerdi, S.G. Bioaccessibility of polyphenols released and associated to dietary fibre in calyces and decoction residues of Roselle (Hibiscus sabdariffa L.). J. Funct. Foods 2015, 18, 171-181. [CrossRef]

126. Hassan, S.T.S.; Švajdlenka, E. Biological evaluation and molecular docking of protocatechuic acid from Hibiscus sabdariffa L. as a potent urease inhibitor by an ESI-MS based method. Molecules 2017, 22, 1696. [CrossRef]

127. Chin, K.L.; Zhen, J.; Qi, Y.; Chin, S.L.; Breithaupt, M.; Wu, Q.L.; Simon, J.; Henson, J.; Ferchaud, V. A comparative evaluation: Phytochemical composition and antioxidant capacity of three roselle (Hibiscus sabdariffa L.) accessions. Acta Hortic. 2016, 1125, 99-107. [CrossRef]

128. Amaya-Cruz, D.; Peréz-Ramírez, I.F.; Pérez-Jiménez, J.; Nava, G.M.; Reynoso-Camacho, R. Comparison of the bioactive potential of Roselle (Hibiscus sabdariffa L.) calyx and its by-product: Phenolic characterization by UPLC-QTOF MSE and their anti-obesity effect in vivo. Food Res. Int. 2019, 126. [CrossRef] [PubMed] 
129. Widowati, W.; Rani, A.P.; Amir Hamzah, R.; Arumwardana, S.; Afifah, E.; Kusuma, H.S.W.; Rihibiha, D.D.; Nufus, H.; Amalia, A. Antioxidant and antiaging assays of Hibiscus sabdariffa extract and its compounds. Nat. Prod. Sci. 2017, 23, 192-200. [CrossRef]

130. Zannou, O.; Kelebek, H.; Selli, S. Elucidation of key odorants in Beninese Roselle (Hibiscus sabdariffa L.) infusions prepared by hot and cold brewing. Food Res. Int. 2020, 133, 109133. [CrossRef]

131. Juhari, N.H.; Petersen, M.A. Physicochemical properties and oxidative storage stability of milled roselle (Hibiscus sabdariffa L.) seeds. Molecules 2018, 23, 385. [CrossRef]

132. Abdel-shafi, S.; Al-Mohammadi, A.-R.; Sitohy, M.; Mosa, B.; Ismaiel, A.; Enan, G.; Osman, A. Antimicrobial Activity and Chemical Constitution of the Crude, Phenolic-Rich Extracts of Hibiscus sabdariffa, Brassica oleracea and Beta vulgaris. Molecules 2019, 24, 4280. [CrossRef] [PubMed]

133. Kane, A.; Achir, N.; Cisse, M.; Pallet, D.; Sakho, M.; Dornier, M. Identification of roselle varieties through simple discriminating physicochemical characteristics using multivariate analysis. Food Sci. Technol. 2019, 39, 321-327. [CrossRef]

134. Sukkhaeng, S.; Promdang, S.; Doung-ngern, U. Fruit characters and physico-chemical properties of roselle (Hibiscus sabdariffa L.) in Thailand-A screening of 13 new genotypes. J. Appl. Res. Med. Aromat. Plants 2018, 11, 47-53. [CrossRef]

135. Soradech, S.; Kusolkumbot, P.; Reungpatthanaphong, P.; Thubthimthed, S. Investigation of DPPH radical scavenging, antioxidant and melanogenesis stimulating activities of various pigment extracts from Thai herbal plants. Res. J. Pharm. Biol. Chem. Sci. 2016, 7, 392-399.

136. Bi, W.; He, C.; Ma, Y.; Shen, J.; Zhang, L.H.; Peng, Y.; Xiao, P. Investigation of free amino acid, total phenolics, antioxidant activity and purine alkaloids to assess the health properties of non-Camellia tea. Acta Pharm. Sin. B 2016, 6, 170-181. [CrossRef] [PubMed]

137. Bayram, O.; Sagdic, O.; Ekici, L. Natural food colorants and bioactive extracts from some edible flowers. J. Appl. Bot. Food Qual. 2015, 88, 170-176. [CrossRef]

138. Aishah, B.; Nursabrina, M.; Noriham, A.; Norizzah, A.R.; Mohamad Shahrimi, H. Anthocyanins from Hibiscus sabdariffa, melastoma Malabathricum and Ipomoea batatas and its color properties. Int. Food Res. J. 2013, 20, 827-834.

139. Chunthanom, P.; Chaikham, P.; Intaket, R. Biochemical and antibacterial properties of Thai medicine herbal infusions. Int. Food Res. J. 2013, 20, 1901-1907.

140. Singh, S.; Singh, D.R.; Salim, K.M.; Srivastava, A.; Singh, L.B.; Srivastava, R.C. Estimation of proximate composition, micronutrients and phytochemical compounds in traditional vegetables from Andaman and Nicobar Islands. Int. J. Food Sci. Nutr. 2011, 62, 765-773. [CrossRef]

141. Rezende, F.A.G.G.; Sande, D.; Coelho, A.C.; Oliveira, G.P.; Boaventura, M.A.D.; Takahashi, J.A. Edible flowers as innovative ingredients for future food development: Anti-Alzheimer, antimicrobial and antioxidant potential. Chem. Eng. Trans. 2019, 75, 337-342. [CrossRef]

142. Sánchez-Feria, C.; González-Hernández, V.A.; Salinas-Moreno, Y.; Cruz-Huerta, N. Genotype and Environmental Effects on Physical and Chemical Qualities of Mexican Varieties of Hibiscus sabdariffa L. Flowers 2017, 51, 525-541.

143. Kashyap, D.; Sarmah, P.; Sarma, A. Nutritional evaluation of calyces and epi-calyces from two variants of Hibiscus sabdariffa l.: A comparison. Res. J. Med. Plant. 2015, 9, 241-247. [CrossRef]

144. Sáyago-Ayerdi, S.G.; Velázquez-López, C.; Montalvo-González, E.; Goñi, I. By-product from decoction process of Hibiscus sabdariffa L. calyces as a source of polyphenols and dietary fiber. J. Sci. Food Agric. 2014, 94, 898-904. [CrossRef] [PubMed]

145. Formagio, A.; Ramos, D.; Vieira, M.; Ramalho, S.; Silva, M.; Zárate, N.; Foglio, M.; Carvalho, J. Phenolic compounds of Hibiscus sabdariffa and influence of organic residues on its antioxidant and antitumoral properties. Brazilian J. Biol. 2015, 75, 69-76. [CrossRef] [PubMed]

146. Loyola Arenas, K.S.; Cruz Y Victoria, M.T.; Vizcarra Mendoza, M.G.; Martínez Vera, C.; Anaya Sosa, I. Effect of agitated bed drying on the retention of phenolic compounds, anthocyanins and antioxidant activity of roselle (Hibiscus sabdariffa L.). Int. J. Food Sci. Technol. 2016, 51, 1457-1464. [CrossRef]

147. Kalla, M.L.M.; Jong, E.N.; Kayem, J.G.; Sreekumar, M.M.; Nisha, P. Effect of re-extraction parameters and drying temperature on the antioxidant properties and dietary fiber of Red sorrel (Hibiscus sabdariffa L.) calyces residues. Ind. Crops Prod. 2015, 74, 680-688. [CrossRef]

148. Leyva Daniel, D.E.; Barragán Huerta, B.E.; Vizcarra Mendoza, M.G.; Anaya Sosa, I. Effect of drying conditions on the retention of phenolic compounds, anthocyanins and antioxidant activity of roselle (Hibiscus sabdariffa L.) added to yogurt. Int. J. Food Sci. Technol. 2013, 48, 2283-2291. [CrossRef]

149. Paraíso, C.M.; dos Santos, S.S.; Ogawa, C.Y.L.; Sato, F.; dos Santos, O.A.A.; Madrona, G.S. Hibiscus sabdariffa L. extract: Characterization (FTIR-ATR), storage stability and food application. Emirates J. Food Agric. 2020, 32, 55-61. [CrossRef]

150. Maldonado-Astudillo, Y.I.; Jiménez-Hernández, J.; Arámbula-Villa, G.; Flores-Casamayor, V.; Álvarez-Fitz, P.; Ramírez-Ruano, M.; Salazar, R. Effect of water activity on extractable polyphenols and some physical properties of Hibiscus sabdariffa L. calyces. J. Food Meas. Charact. 2019, 13, 687-696. [CrossRef]

151. Mollah, M.A.F.; Tareq, M.Z.; Bashar, K.K.; Zahidul Hoque, A.B.M.; Karim, M.M.; Al Rafiq, M.Z. Antioxidant properties of BJRI vegetable mesta-1 (Hibiscus sabdariffa L.). Plant. Sci. Today 2020, 7, 154-156. [CrossRef]

152. Apáez Barrios, P.; Granados, M.D.C.R.; Santos, M.E.P.; Montaño, Y.A.R. Effect of foliar copper application on yield and anthocyanin concentration in Hibiscus sabdariffa calyces. Rev. Fac. Ciencias Agrar. 2018, 50, 65-75.

153. Ibrahim, R.; Mazuki, N.A.F. The quality of roselle (Hibiscus sabdariffa L.) juices made from roselle calyces stored at different cold temperatures. Malaysian Appl. Biol. 2013, 42, 67-71. 
154. Bechoff, A.; Cissé, M.; Fliedel, G.; Declemy, A.L.; Ayessou, N.; Akissoe, N.; Touré, C.; Bennett, B.; Pintado, M.; Pallet, D.; et al. Relationships between anthocyanins and other compounds and sensory acceptability of Hibiscus drinks. Food Chem. 2014, 148, 112-119. [CrossRef] [PubMed]

155. Wu, H.Y.; Yang, K.M.; Chiang, P.Y. Roselle anthocyanins: Antioxidant properties and stability to heat and pH. Molecules 2018, 23, 1357. [CrossRef] [PubMed]

156. Alarcón-Alonso, J.; Zamilpa, A.; Aguilar, F.A.; Herrera-Ruiz, M.; Tortoriello, J.; Jimenez-Ferrer, E. Pharmacological characterization of the diuretic effect of Hibiscus sabdariffa Linn (Malvaceae) extract. J. Ethnopharmacol. 2012, 139, 751-756. [CrossRef]

157. Nuryanti, S.; Matsjeh, S.; Anwar, C.; Raharjo, T.J. Isolation anthocyanin from roselle petals (Hibiscus sabdariffa L) and the effect of light on the stability. Indones. J. Chem. 2012, 12, 167-171. [CrossRef]

158. Pulgarín, J.A.M.; Bermejo, L.F.G.; Durán, A.C. A fast and simple FIA-chemiluminescence method for the evaluation of Roselle flowers as scavenger of the free radicals generated by UV irradiated antibiotics. J. Pharm. Biomed. Anal. 2019, 164, 630-635. [CrossRef]

159. Takahashi, J.A.; Rezende, F.A.G.G.; Moura, M.A.F.; Dominguete, L.C.B.; Sande, D. Edible flowers: Bioactive profile and its potential to be used in food development. Food Res. Int. 2020, 129. [CrossRef] [PubMed]

160. Brewer, M.S. Natural Antioxidants: Sources, Compounds, Mechanisms of Action, and Potential Applications. Compr. Rev. Food Sci. Food Saf. 2011, 10, 221-247. [CrossRef]

161. Tahir, H.E.; Xiaobo, Z.; Jiyong, S.; Mariod, A.A.; Wiliam, T. Rapid Determination of Antioxidant Compounds and Antioxidant Activity of Sudanese Karkade (Hibiscus sabdariffa L.) Using Near Infrared Spectroscopy. Food Anal. Methods 2016, 9, 1228-1236. [CrossRef]

162. Ilyasov, I.R.; Beloborodov, V.L.; Selivanova, I.A.; Terekhov, R.P. ABTS/PP decolorization assay of antioxidant capacity reaction pathways. Int. J. Mol. Sci. 2020, 21, 1131. [CrossRef] [PubMed]

163. Ademiluyi, A.O.; Oboh, G. Aqueous extracts of roselle (Hibiscus sabdariffa Linn.) varieties inhibit $\alpha$-amylase and $\alpha$-glucosidase activities in vitro. J. Med. Food 2013, 16, 88-93. [CrossRef] [PubMed]

164. Higginbotham, K.L.; Burris, K.P.; Zivanovic, S.; Davidson, P.M.; Stewart, C.N. Aqueous extracts of Hibiscus sabdariffa calyces as an antimicrobial rinse on hot dogs against Listeria monocytogenes and methicillin-resistant Staphylococcus aureus. Food Control. 2014, 40, 274-277. [CrossRef] 